Landslide path dependency and its impact on space-time modelling of landslide susceptibility

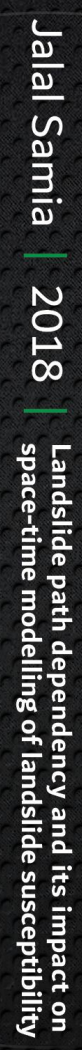

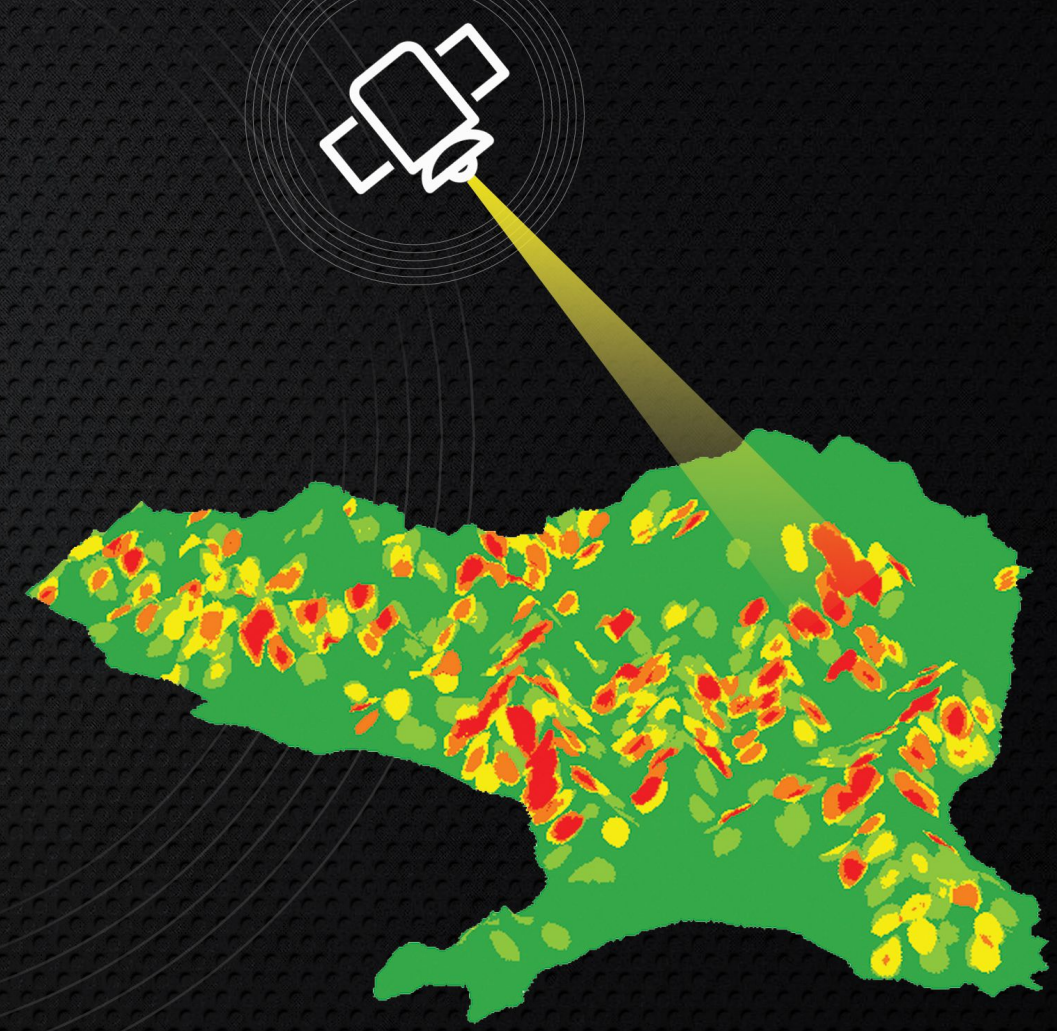

Jalal Samia 


\section{Propositions}

1. Landslide susceptibility models must take landslide path dependency into account. (this thesis)

2. Landslide susceptibility is dynamic on a decadal time scale. (this thesis)

3. Food security is further ensured by sustainable thinking.

4. Most natural hazards are the consequence of human interference.

5. Political conflicts should not hamper international travel by scientists.

6. Signed agreements between nations should be adhered to when governments change.

Propositions belonging to the thesis of Jalal Samia

Landslide path dependency and its impact on space-time modelling of landslide susceptibility

Wageningen, 2 November 2018 
Landslide path dependency and its impact on space-time modelling of landslide susceptibility

\author{
Jalal Samia
}




\section{Thesis committee}

\section{Promotors}

Prof. Dr A. K. Bregt

Professor of Geo-Information Science

Wageningen University \& Research

Prof. Dr J. Wallinga

Professor of Soil Geography and Landscape

Wageningen University \& Research

\section{Co-promotor}

Dr A. J. A. M. Temme

Associate Professor, Department of Geography

Kansas State University, United States of America

\section{Other members}

Dr P. Reichenbach, CNR IRPI, Italy

Prof. H-B. Havenith, University of Liege, Belgium

Dr C. J. Van Westen, University of Twente, The Netherlands

Prof. Dr CJ. Ritsema, Wageningen University and Research

This research was conducted under the auspices of the C.T. de Wit Graduate School of Production Ecology \& Resource Conservation (PE\&RC) 


\title{
Landslide path dependency and its impact on space-time modelling of landslide susceptibility
}

\author{
Jalal Samia
}

\section{Thesis}

submitted in fulfilment of the requirements for the degree of doctor at Wageningen University

by the authority of the Rector Magnificus,

Prof. Dr A.P.J. Mol, in the presence of the

Thesis Committee appointed by the Academic Board to be defended in public on Friday 2 November 2018 at 11 a.m. in the Aula. 
Jalal Samia

Landslide path dependency and its impact on space-time modelling of landslide susceptibility

$\mathrm{PhD}$ thesis, Wageningen University, Wageningen, the Netherlands (2018)

With references, with summary in English

ISBN: 978-94-6343-514-7

DOI: $10.18174 / 459815$ 


\section{Contents}

Page

Chapter 1. Introduction 1

Chapter 2. Do landslides follow landslides? Insights in path dependency from a multi-temporal landslide inventory

Chapter 3. Characterization and quantification of path dependency in landslide susceptibility

Chapter 4. Implemeting landslide path dependency in landslide susceptibility modelling

Chapter 5. Pixel-based dynamic path dependent landslide susceptibility modelling

Chapter 6. Synthesis

References

118

Summary

Acknowledgements

List of publications

Short biography

PE\&RC Training and Education Statement 



\section{Chapter 1}

Introduction 


\subsection{Introduction}

The term 'landslide' represents the movement of a mass of rock, debris, or earth down a slope, under the influence of gravity (Cruden 1996). Landslides can be classified into fall, topples, slide, flow, spread and complex landslides, based on the type of movement (Figure 1) (Varnes 1978; Crozier 1986; Cruden 1996). Complex landslides contain a combination of two or more principle types of movements at the same time, during the evolution of the landslide.

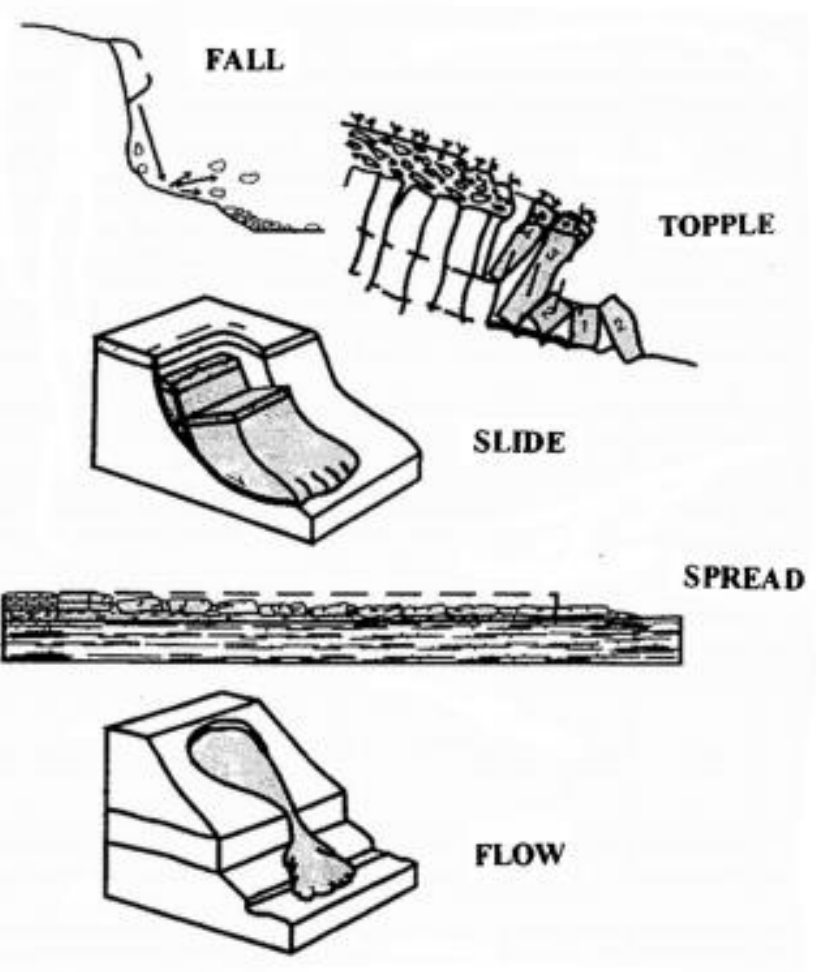

Figure 1.1. Classification of landslides according to the type of movement (Cruden 1996)

Intense or prolonged rainfall, earthquakes, and rapid snowmelt are the main external triggers in areas susceptible to landslides. Also, the intensive exploitation of land by humans for building infrastructure (i.e., railway and road construction, new settlements) are increasing the occurrence of landslides in landslide prone areas (Guzzetti 2006).

Landslides are geo-hydromorphological natural events that have disastrous impacts on society and the environment. They cause fatalities and economic losses with negative long-term socio-economic effects. Petley (2012) analyzed the global 
pattern of fatalities that resulted from landslides. He concluded that from 2004 to 2010, 2620 fatal landslides occurred globally with a total of 32,322 recorded deaths. Also, regarding the economic losses from landslides, Brabb (1991) assessed that the annual economic losses and damages arising from landslides could be tens of billions of dollars. The impacts of landslides on the environment can be longlasting. Landslides change the topography of earth surface, the structure of rivers and streams and quality of water, cause deforestation and change the habitat of natural wildlife (Geertsema et al. 2009).

\subsection{Landslide inventory maps}

The spatial distribution of landslides are documented in landslide inventory maps. A comprehensive database for a landslide inventory map should contain information on landslides that have left recognizable signs in the landscape. The required information are the location and time of landslide occurrence (or time of mapping), and the size, extent and type of the landslide (Guzzetti et al. 2000; Guzzetti et al. 2012). The distribution of landslides in most landslide inventory maps were traditionally recorded in point format. After the introduction of GIS, landslides are documented in polygon format (Xu 2015). Nowadays, the multi-stage activities of landslides can be monitored and mapped over several decades using time series of remote sensing measurements (Geertsema et al. 2009).

Several factors influence the mapping and documenting of landslides: i) the applicability of landslide inventory maps, ii) the scale of base maps, iii) the scale and resolution of imagery sources (e.g., aerial photographs, remote sensing satellite images and LiDAR elevation data), and skills and experience of geomorphologists in identifying landslides through iv) imageries and v) direct field mapping (Guzzetti et al. 2000; Van Westen et al. 2006; Guzzetti et al. 2012). Landslide inventories are prepared usually on three different scales, from small-scale inventories $(<1: 200,000)$ and medium-scale inventories $(<1: 25,000$ to $1: 200,000)$ to largescale inventories (>1:25,000) (Guzzetti et al. 2012). Also, depending on the type of mapping, landslide inventory maps are grouped into archive mapping and geomorphological mapping (Guzzetti et al. 2000; Malamud et al. 2004). In archive mapping, landslide information is collected from news, newspapers, articles and other literature sources (Taylor and Brabb 1986). Geomorphological landslide inventory maps are classified into three groups: i) historical inventories which show landslides over periods of tens to thousands of years (Galli et al. 2008), ii) event-based inventories which document single landslides after external triggers such as rainfall events (Guzzetti et al. 2004) and earthquakes (Parker et al. 2011), and iii) multi-temporal landslide inventories which map landslides over longer time periods (Galli et al. 2008). 


\subsection{Landslide susceptibility modelling}

To mitigate the destructive effects of landslides, decision makers and land use planners use landslide susceptibility models and maps, which reflect the likelihood of landslide occurrence based on local terrain properties (Brabb 1984). The term 'landslide susceptibility' refers to 'where' landslides are likely to occur (Guzzetti et al. 2005; Guzzetti et al. 2006). In mathematical words, landslide susceptibility reflects the spatial probability of landslide occurrence given a set of environmental conditioning attributes (Guzzetti et al. 2005). This separates landslide susceptibility as a purely spatial concept from landslide hazard in two aspects (Guzzetti et al. 2005; Guzzetti et al. 2006; Rossi et al. 2010): landslide susceptibility is time-invariant and does not take the temporal probability of landslide occurrence into account. Also, it does not consider the magnitude of expected landslides (i.e., how large or destructive the landslide occurrence will be). Both landslide inventory mapping and landslide susceptibility modelling are the main initial and crucial steps towards landslide hazard and risk assessment (Cardinali et al. 2002; Guzzetti et al. 2005; Van Westen et al. 2006). In landslide hazard, the spatial susceptibility is combined with temporal information of landslide triggers (mainly rainfall), providing the information regarding 'when' or how frequently landslides will occur and with which magnitude (Guzzetti et al. 2005). The combination of landslide mapping, landslide susceptibility modelling and landslide hazard modelling contribute to the assessment of landslide risk, which reflects the expected number of casualties, injuries, and the economic losses to society and the environment (Varnes 1984; Van Westen et al. 2006). These are the key tools towards the sustainable planning of land use, proper decision making, and the mitigation of destructive impacts of landslides.

In the two last decades, there has been much progress in the development of opensource and commercial GIS and statistical software, and an increased availability of environmental data in GIS databases. This has led to many different approaches and techniques for modelling susceptibility to landslide occurrence (Rossi et al. 2010). These approaches and techniques can be both qualitative and quantitative. Qualitative approaches rely on the experience and knowledge of geomorphologists, and subjectively describe the susceptibility levels in qualitative terms (Van Westen et al. 2003; Reichenbach et al. 2018). Quantitative approaches combine the landslide distribution from the landslide inventories with environmental conditioning attributes to model susceptibility to landslide occurrence (Van Westen et al. 2006). Reichenbach et al. (2018) classified all the approaches in the literature into five groups: (i) geomorphological mapping, (ii) analysis of landslide distribution in landslide inventories, (iii) heuristic or index-based approaches (iv), physically or process-based models, and (v) statistically-based models. These approaches all rely on the same assumption to model landslide susceptibility: the past and present are key to understand the future (Varnes 1984; Carrara et al. 1991; Hutchinson 1995). From this assumption follows that landslides will occur in the future under the same conditions that they occurred under in the past and present. 


\subsection{Research gaps}

In the last two decades, rise and development in GIS, remote sensing and statistical software have facilitated collecting, mapping and modelling susceptibility of landslides. Using these novel technologies and data, landslide susceptibility modelling at medium scale has become playground for many researchers to try new data integration approaches and techniques. This has resulted in the many different qualitative and quantitative approaches for landslide susceptibility modelling. However, due to complex nature of landsliding, the prediction of the occurrence of landslides in both space and time is still difficult (Deng et al. 2017).

The underlying assumption in different landslide susceptibility modelling approaches is 'past and present are the key to the future' (Varnes 1984; Carrara et al. 1991). In such an assumption, usually relations between environmental factors and distribution of landslides are considered. Importantly, this assumption does not consider possible legacy effects of landslides on the environment, and its effects on the future landslide susceptibility.

Yet, these legacy effects of landslides on environmental variables are expected since landslides disrupt the morphology of surface and topography, slope angle (Van Westen et al. 2006) and reduce the strength of materials forming the hillslope. This implicates that the legacy of landslides could change the future landslide susceptibility. However, changes caused by landslides in the environmental variables are difficult to obtain and not usually available since they need continuous monitoring and mapping of environmental variables.

A path dependency horizon from complex system theory (Phillips 2006) could be used to reflect the historical legacy effects of landslides on the environment, and hence on the future susceptibility. In this respect, path dependency means that the history of a system determines the future state of the system through legacy effects. In other words, previous landslides could trigger future landslides. The reason for neglecting such an effect could be the lack of mapping and monitoring of multi-stage activities of landslides. Multi-temporal landslide inventory does allow monitoring and mapping of multi-stage activities, evolution and legacy effects of landslides. In this research, a multi-temporal landslide inventory - provided by CNR-IRPI from Collazzone study area in Umbria region in central of Italy - is used. This multitemporal landslide inventory contains mapped landslides in 19 time slices over a period of 75 years. 


\section{$1.5 \quad$ Research objectives}

The overall objective of this thesis is to assess the effect of landslides on susceptibility of future landslides. To explore such an effect, the following research questions are addressed:

A. what are the indications of landslides following previous landslides?

B. What are the characteristic spatial and temporal scales in landslide path dependency?

C. Can we predict the occurrence of future landslides as a function of properties of previous landslides?

D. Does including landslide path dependency improve landslide susceptibility modelling?

\subsection{Thesis outline}

The chapters 2, 3 and 4 of this thesis are based on three peer-reviewed publications, and the chapter 5 is based on a manuscript to be submitted. These chapters deal with one or two research questions posed in the introduction as indicated in Table 1.1 .

Table 1.1. Research questions and chapters.

\begin{tabular}{cc}
\hline Research question & Chapter \\
\hline A & 2 \\
B & 2,3 and 5 \\
C & 3 \\
D & 4,5 \\
\hline
\end{tabular}

Chapter 2 explores the existence of path dependency among landslides using empirical approaches and the index of spatial overlap of landslides in the Collazzone multi-temporal landslide inventory. I also looked at the differences in the size, shape and parameters of frequency-area statistics between landslides with spatial overlap and landslides without spatial overlap. At the end, I propose a modification in the definition of landslide susceptibility modelling; to take landslide path dependency effects into account.

Chapter 3 characterizes and quantifies the spatiotemporal dynamics of landslide path dependency in the multi-temporal landslide inventory. The geometrical and topographical differences between landslides that cause or do not cause follow-up 
landslides are assessed. Also, I tried to predict the occurrence of a follow-up landslide using geometric and topographic attributes of an earlier landslide.

Chapter 4 implements the effect of landslide path dependency in slope-based landslide susceptibility modelling. I developed three different landslide susceptibility models using logistic regression: i) a conventional landslide susceptibility model, ii) a conventional plus path dependent landslide susceptibility model, and iii) a pure path dependent landslide susceptibility model. Finally, I compared the coefficients, performance and susceptibility maps from these three models.

Chapter 5 quantifies spatiotemporal dynamics of landslide path dependency between the center points of landslides using space-time Ripley's K Function. Then, the quantified space-time landslide path dependency is implemented in a pixelbased landslide susceptibility model and the result is compared to a conventional landslide susceptibility model and a purely path dependent landslide susceptibility model. The performance, coefficients and landslide susceptibility maps from the three models are compared.

Chapter 6 summarizes the main findings of this thesis and provides reflections, outlooks and recommendations for further research in this field.

Polygon-based multi-temporal inventory

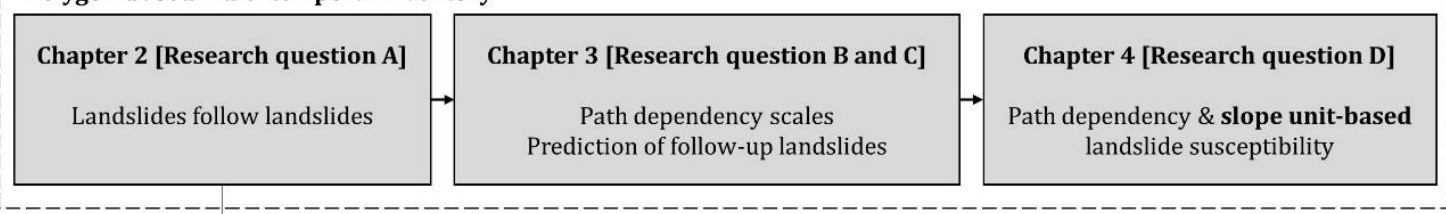

Point-based multi-temporal inventory

Chapter 5 [Research question D]

Path dependency \& pixel-based landslide susceptibility

Figure1.2. Overview of the four core chapters of this thesis and how they are linked. 



\section{Chapter 2}

\section{Do landslides follow landslides? Insights in path dependency from a multi-temporal landslide inventory}

This chapter is based on:

Samia, J., Temme, A., Bregt, A., Wallinga, J., Guzzetti, F., Ardizonne, F., Rossi, M. (2017). Do landslides follow landslides? Insights in path dependency from a multitemporal landslide inventory. Landslides, 14, 547-558, DOI: 10.1007/s10346-0160739-x 


\begin{abstract}
Landslides are a major category of natural disasters, causing loss of lives, livelihoods and property. The critical roles played by triggering (such as extreme rainfall and earthquakes) and intrinsic factors (such as slope steepness, soil properties and lithology) have previously successfully been recognized and quantified using a variety of qualitative, quantitative and hybrid methods in a wide range of study sites. However, available data typically do not allow to investigate the effect that earlier landslides have on intrinsic factors and hence on follow-up landslides. Therefore, existing methods cannot account for the potentially complex susceptibility changes caused by landslide events. In this study, we used a substantially different alternative approach to shed light on the potential effect of earlier landslides using a multi-temporal dataset of landslide occurrence containing 17 time slices. Spatial overlap and the time interval between landslides play key roles in our work. We quantified the degree to which landslides preferentially occur in locations where landslides occurred previously, how long such an effect is noticeable, and how landslides are spatially associated over time. We also investigated whether overlap with previous landslides causes differences in landslide geometric properties. We found that overlap among landslides demonstrates a clear legacy effect (path dependency) that has influence on the landslide affected area. Landslides appear to cause greater susceptibility for followup landslides over a period of about ten years. Follow-up landslides are on average larger and rounder than landslides that do not follow earlier slides. The effect of earlier landslides on follow-up landslides has implications for understanding of the landslides evolution and the assessment of landslide susceptibility.
\end{abstract}




\subsection{Introduction}

Existing landslide research recognizes the critical role that is played by external triggers (e.g. extreme rainfall events, earthquakes and human interferences) along with intrinsic attributes of site (e.g. slope and lithology) that contribute to landslide occurrence (Carrara et al. 1999; Crozier 1986; Guzzetti et al. 2008). Much work has been done on the modelling of landslide susceptibility and hazard. By definition, landslide susceptibility is a non-temporal concept that refers to locations where landslides preferentially occur (Guzzetti et al. 2005; Varnes 1984), whereas landslide hazard describes the likelihood of landslide occurrence in time and space (Guzzetti et al. 1999; Varnes 1984) along with the magnitude of landslide occurrence (Guzzetti et al. 1999). Landslide susceptibility and hazard have been studied using qualitative (Barredo et al. 2000; Ruff and Czurda 2008; Van Westen et al. 2003), and quantitative (Godt et al. 2008; Guzzetti et al. 2005; Lan et al. 2004; Remondo et al. 2003; Van Westen et al. 1997; Van Westen and Terlien 1996; Yeon et al. 2010) approaches. Qualitative approaches emphasise the role of experience and expert knowledge in determining landslide susceptibility (Van Westen et al. 2003). Quantitative approaches assume that conditions that lead to landslide occurrence in the past and present are likely to cause landslides in the future, thus the probability of occurrence of future landslides is determined using correlations among various conditioning factors and landslide inventories by statistical methods (Tien Bui et al. 2015; Van Westen and Terlien 1996). Deterministic quantitative approaches use detailed geotechnical and hydrological data in combination with statistical models to estimate the probability of slope failure (Aleotti and Chowdhury 1999; Van Westen and Terlien 1996).

Empirical landslide inventories that document the location, and sometimes the date, shape and type of landslides play an important role in assessing landslide susceptibility and hazard (Cardinali et al. 2006; Guzzetti et al. 2012; Guzzetti et al. 2005; van Westen et al. 2008; van Westen et al. 2006b). Most of the landslide inventories are prepared through the interpretation of stereoscopic aerial photographs (Guzzetti et al. 2012). In addition, field mapping (Brunsden 1993), analysis of surface morphology through high-resolution DEMs and interpretation of satellite images (Guzzetti et al. 2012) are used for mapping landslide inventories. Overall statistics of landslide inventories have been studied and it was documented that the frequency-area distribution of landslides follows a power law distribution for medium and large landslides with an exponential rollover for small landslides (Guzzetti et al. 2009; Guzzetti et al. 2002; Malamud et al. 2004; Stark and Hovius 2001; Turcotte et al. 2006; Wood et al. 2015).

Although a vast amount of research has been done on the prediction of landslide occurrence, there has, to our knowledge, been no empirical attention for the effect of earlier landslides on future landslides. This effect is nonetheless expected because landslides typically change the surface morphology (Schuster and Highland 2003), the sediment or regolith properties (Chen 2009), the vegetation (Singh et al. 2014) and the slope angle (van Westen et al. 2006a), which are all factors that change landslide susceptibility. If true, such importance of landslide 
history for landslides susceptibility would be a form of path dependency (a concept from complexity theory (Phillips 2006; Temme et al. 2015)) - indicating that the history of the landsliding process affects its future through one or more legacy effects. A likely reason for the lack of attention for quantifying the effect of earlier landslides on future landslides is that multi-temporal landslide inventories are very difficult to obtain (Atkinson and Massari 1998; Brenning 2005) and high-resolution multi-temporal datasets of intrinsic properties are virtually absent.

In this paper, we explore the possible effects of earlier landslides on future landslides. For this, we will use a rich multi-temporal landslide inventory from the Collazzone area in central Umbria, Italy (Ardizzone et al. 2013; Galli et al. 2008; Guzzetti et al. 2006).

Our main objective is to investigate whether earlier landslides determine the susceptibility for future landslides, i.e. whether there is path dependency in landslide occurrence. We test two hypotheses. The first hypothesis is that landslides follow landslides; i.e., that landslides increase the likelihood of another (in our terms: follow-up) landslide occurring in the same place. We operationalize this hypothesis without attention for the role of intrinsic attributes (e.g., slope and geology) to offer a clear contrast with existing approaches. The second hypothesis is that follow-up landslides differ from other landslides in terms of their shape, size, and frequency-area distribution.

\subsection{Study area and data}

The Collazzone area extends for $78.9 \mathrm{~km}^{2}$ in central Umbria, Italy (Figure 2.1). Elevation in the area ranges from 145 to $634 \mathrm{~m}$ above sea level and the slope, computed from a $10 \times 10 \mathrm{~m}$ Digital Terrain Model (DTM), ranges from $0^{\circ}$ to $63.7^{\circ}$ with a mean value of $9.9^{\circ}$. The terrain is hilly with asymmetrical valleys, with lithology and attitude of bedding controlling the slopes. Only sedimentary rocks crop out in the area and include: (1) alluvial deposits, Holocene in age; (2) travertine, Pleistocene in age; (3) continental deposits (gravel, sand, clay), PlioPleistocene in age (Guzzetti et al. 2006); (4) layered sandstone and marl, Miocene in age; and (5) thinly layered limestone, Lias to Oligocene in age. The land use is characterised by cropland, forests, urban areas, pastures, vineyards, orchards and water. Farming in the area favours the development of slope failures and erosion. Soils range in thickness from a few decimetres to more than $1 \mathrm{~m}$; they have a fine or medium texture (Fiorucci et al. 2015). Soils have a xeric moisture regime characterized by cold and moist winters and dry summers. Precipitation is most abundant in October and November; with a mean annual rainfall of $841 \mathrm{~mm}$ in the period from 1951 to 2013. Snow falls in the area on average every 2-3 years. Landslides are abundant, and range in age, type, morphology, and volume from relict - partly eroded - large and deep-seated landslides, to young, mostly shallow landslides involving the soil mantle. Landslides are triggered predominantly by meteorological events, including intense and prolonged rainfall and rapid snowmelt. 


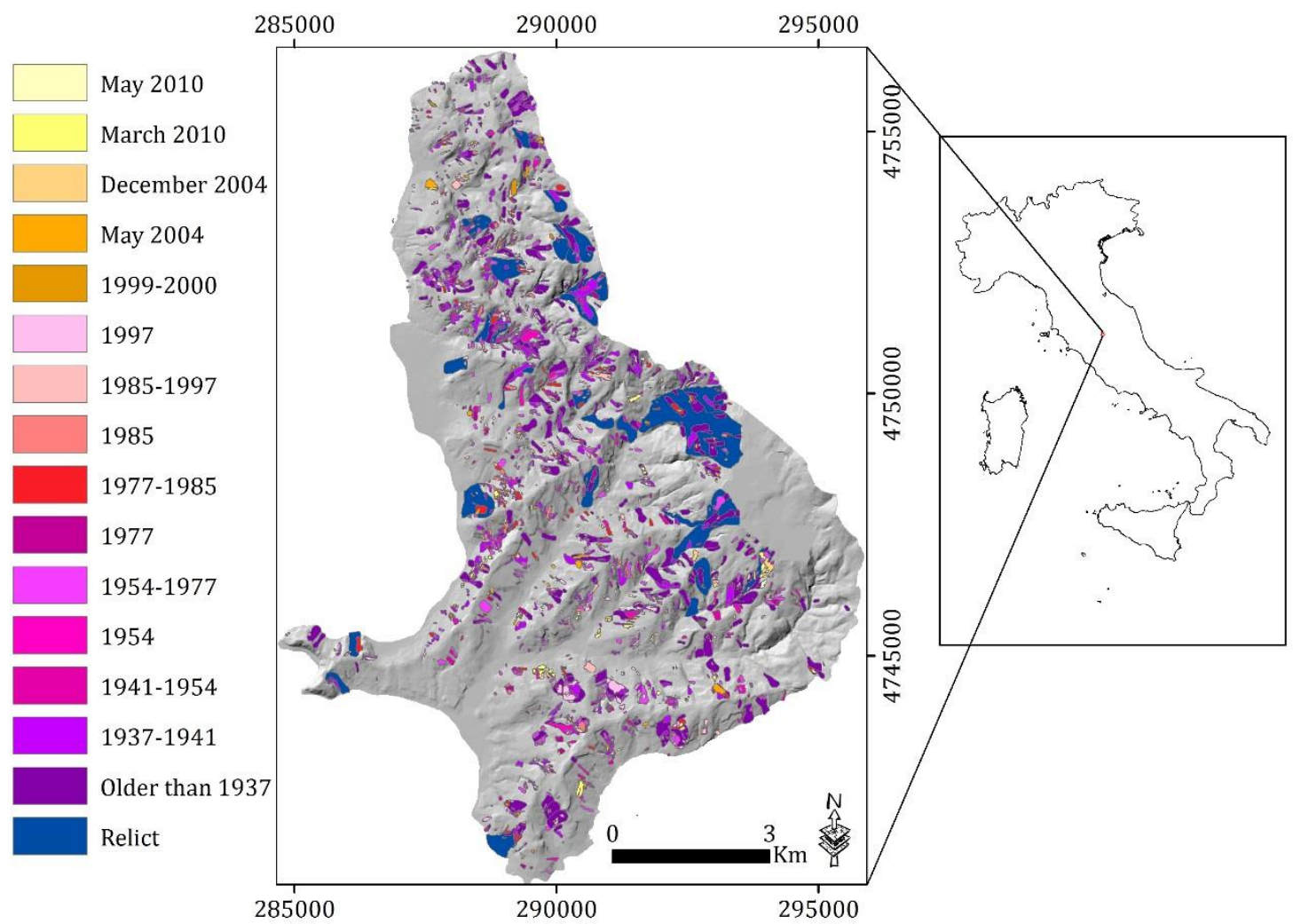

Figure 2.1. Location of Umbria region and of the Collazzone study area (right map). Multitemporal landslide inventory overlying a shaded relief image (left map). The coordinate system of the relief image is EPSG:32633.

\subsubsection{Description of the Multi-temporal Landslide Inventory}

A detailed multi-temporal landslide inventory is available for the Collazzone area (Figure 2.1and Table 2.1). The inventory was originally prepared at 1:10,000 scale through the visual interpretation of five sets of stereoscopic aerial photographs taken unsystematically in the period 1941-1997 at scales ranging from 1:13,000 to 1:33,000. The landslide inventory was continued in the period from 1999 to December 2005 through field surveys carried out after periods of prolonged rainfall, and in March and May 2010 using stereo satellite images (Ardizzone et al. 2013).

Landslides ages were estimated from the date of the aerial photographs and the morphological appearance of the landslide. In each of the five sets of stereoscopic aerial photographs used to prepare the multi-temporal inventory, landslides that appeared "fresh" on the aerial photographs, were separated from the other landslides that had occurred since the previous photograph. The date (i.e., year) of the aerial photographs used to identify the landslides was assigned to the "fresh" 
slope failures. The other slope failures (i.e., the "non-fresh" landslides) were attributed to the period between the date of the aerial photograph where they were identified and the date of the previous aerial photographs.

Galli et al. (2008) demonstrated the high quality of the multi-temporal landslide inventory map for the Collazzone study area. Nonetheless, we acknowledge that the mapping and age attribution is not perfect. Errors are probably largest in time slices that were obtained through field mapping due to the difficulty in translating lateral assessments onto vertical photographs, but even here we expect the uncertainty in determining a landslide boundary to be less than 3 meters. For other time slices, the aerial photographs and satellite images that were used have resolutions of $1 \mathrm{~m}$ or better, and hence uncertainty is of the same order. With an average size of landslides of over $5000 \mathrm{~m}^{2}$, it was expected that the uncertainty in mapping did not substantially affect our results. 
Table 2.1. Properties of landslides in different time slices of the multitemporal landslide inventory for the Collazzone area, Umbria, Central Italy. Period covered, number and density of landslide per period, total affected area and inventory type per period. Inventory type: $\mathrm{P}$, obtained from the systematic interpretation of stereoscopic aerial photographs; F, obtained through direct field mapping; $S$, obtained from the interpretation of stereoscopic satellite images. Landslide periods are estimated from the date of the aerial photographs and the morphological appearance of the landslide.

\begin{tabular}{|c|c|c|c|c|}
\hline $\begin{array}{l}\text { Period } \\
\text { covered }\end{array}$ & $\begin{array}{l}\text { Number of } \\
\text { landslides(N) }\end{array}$ & $\operatorname{Density}(\mathrm{N}) /\left(\mathrm{Km}^{2}\right)$ & $\begin{array}{r}\text { Total Area of } \\
\text { landslides }\left(\mathrm{Km}^{2}\right)\end{array}$ & $\begin{array}{l}\text { Inventory } \\
\text { type }\end{array}$ \\
\hline Relict & 27 & 0.34 & 5.72 & $\mathrm{P}$ \\
\hline $\begin{array}{c}\text { Older than } \\
1937\end{array}$ & 269 & 3.41 & 6.55 & $\mathrm{P}$ \\
\hline 1937-1941 & 706 & 8.86 & 4.09 & $\mathrm{P}$ \\
\hline 1941-1954 & 63 & 0.79 & 0.42 & $\mathrm{P}$ \\
\hline 1954 & 97 & 1.23 & 0.71 & $\mathrm{P}$ \\
\hline 1954-1977 & 409 & 5.18 & 1.49 & $\mathrm{P}$ \\
\hline 1977 & 252 & 3.19 & 0.69 & $\mathrm{P}$ \\
\hline 1978-1985 & 105 & 1.33 & 0.62 & $\mathrm{P}$ \\
\hline 1985 & 135 & 1.71 & 0.45 & $\mathrm{P}$ \\
\hline 1986-1997 & 63 & 0.79 & 0.27 & $\mathrm{P}$ \\
\hline 1997 & 413 & 5.23 & 0.78 & $\mathrm{P}$ \\
\hline 1999-2000 & 17 & 0.21 & 0.07 & $\mathrm{~F}$ \\
\hline May 2004 & 71 & 0.89 & 0.27 & $\mathrm{~F}$ \\
\hline $\begin{array}{c}\text { December } \\
2004\end{array}$ & 154 & 1.94 & 0.38 & $\mathrm{~F}$ \\
\hline $\begin{array}{c}\text { December } \\
2005\end{array}$ & 62 & 0.90 & 0.18 & $\mathrm{~F}$ \\
\hline March 2010 & 158 & 2.00 & 0.29 & S \\
\hline May 2010 & 55 & 0.69 & 0.085 & S \\
\hline
\end{tabular}




\subsection{Methods}

In addition to our use of the term path dependency (indicating that previous process activity affects future process activity through legacy effects), it is useful to clarify some other terms that are important for our analysis. The mono-temporal inventories that together make up our multi-temporal inventory are separately called 'time-slices', whereas subdivisions of the multi-temporal inventory based on topology (see below) are called 'sub-inventories'. We use 'earlier' and 'follow-up' to describe landslides based on their relative order in the multi-temporal inventory. Note that follow-up landslides are not reactivated landslides. We consider a landslide a reactivated landslide when all or most of the landslide moved down again, under the same general condition as the first landslide. Instead, a follow-up landslides is a new landslides that has different size and shape than the pre-existing landslide. The particular topological relations of interest between landslides in earlier and follow-up time slices we call 'spatial association'.

To test our hypotheses, two sets of analyses were done on the multi-temporal landslide inventory. The first set of analyses focusses on the effect of overlap between landslides on the total area affected by landslides (Figure 2.2a), on the degree of overlap between landslides from different time slices (Figure 2.2b) , and on the number of overlaps between landslides over the entire inventory (Figure 2.2c). The second set of analyses focusses on the properties of classes of landslides that vary in their spatial association with landslides from the earlier periods (Figure $2.2 \mathrm{~d})$. 
Hypothesis 1:

landslides follow landslides

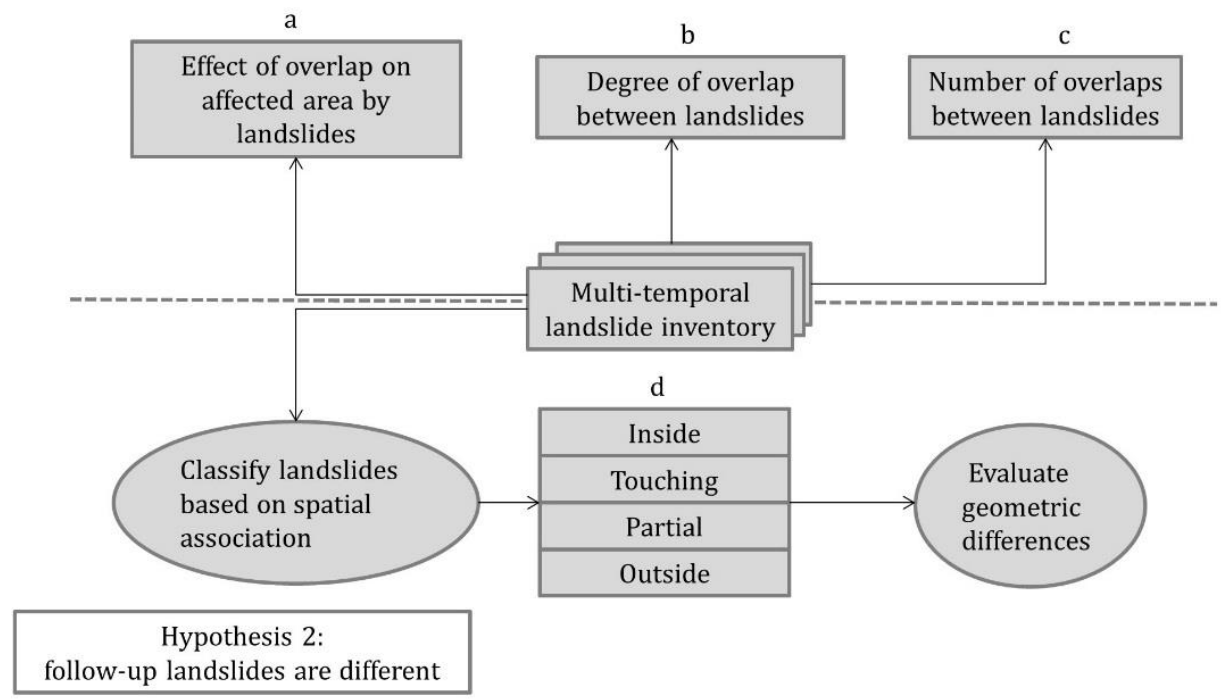

Figure 2.2. Overview of the analyses performed on the multi-temporal dataset to test two hypotheses.

\subsubsection{Degree of overlap}

We propose three complementary methods for this purpose, to express the importance of overlap between landslides: (1) unaffected area, (2) overlap index, and (3) number of overlaps (Figure 2.2).

The first method measures the cumulative effect that landslide overlapping has on the total area affected by landslides, by comparing a theoretically unaffected area with the actually unaffected area. This method allows assessment of the amount of reduction in affected area caused by (potentially followed-up) overlapping landslides. The theoretically unaffected relative area $(-$, where - denotes that this is a dimensionless parameter) if no overlap between landslides would occur is defined as:

Theoretically unaffected area $_{t}=\left(\sum_{\mathrm{i}=1}^{\mathrm{t}} A L_{T i}\right) / A S$

Where AS is the area of interest $\left(\mathrm{m}^{2}\right)$, and ALTi is the total area $\left(\mathrm{m}^{2}\right)$ of all landslides in the time slices i of an inventory. The relative area that is actually unaffected by landslides over the entire period is calculated as:

Actually unaffected area $_{t}=\left(\cup_{i=1}^{t} A L_{T i}\right) / \mathrm{AS}$ 
Where $U$ denotes the spatial union among landslides in consecutive time slices, i.e. the total area covered by landslides after accounting for overlaps.

The second method provides a standardized measure of overlap between landslides in consecutive time slices. This overlap index (-), earlier termed relative area overlap (Maruca and Jacquez 2002), is calculated as:

$$
\text { Overlap index }{ }_{t-t+n}=\frac{\mathrm{AL}_{\mathrm{t}} \cap \mathrm{AL}_{\mathrm{t}+\mathrm{n}}}{\mathrm{AL}_{\mathrm{t}}+\mathrm{AL}_{\mathrm{t}+\mathrm{n}}-\left(\mathrm{AL}_{\mathrm{t}} \cap \mathrm{AL}_{\mathrm{t}+\mathrm{n}}\right)}
$$

Where $t$ is the assumed date of occurrence of landslides from a first time slice (see below), and $t+n$ is the average date of occurrence of landslides in a later time slice, and $\cap$ denotes the geometric intersection (i.e., the overlapping area) between two time slices. This is qualitatively similar to the error index that has been used to calculate positional mismatch for pairs of corresponding landslides in the two inventories (Ardizzone et al. 2002; Carrara et al. 1992; Santangelo et al. 2015). The overlap index is non dimensional, ranges from zero (no overlap) to unity (perfect overlap), and is not a function of the size of the study area. The overlap index was calculated for consecutive time slices, and for pairs of time slices that are two or three time slices apart. The value for the overlap index was then related to the time that passed between time slices.

As explained above, in some time slices, there is more uncertainty about the date of occurrence of a landslide than in others. Uncertainty is larger in the time slices that describe longer periods, such as the time slice describing landslides that occurred between 1954 and 1977 (see Table 1). In some other cases, the date of occurrence is relatively well known, for instance in the time slice made of landslides that occurred after the 01-01-1997 rapid snowmelt event. These uncertainties, large or small, propagate into uncertainty about the time elapsed between landslides. We assumed uniform probability distributions of landslides in the time slices that describe periods - meaning that landslides may have happened at any moment during the considered time slice, with equal probability. This is despite the fact that there may have been significant rain, snowfall and snowmelt events in these periods that may have caused landslides to be clustered in time. No information about this intra-period variability was available. Using the uniform probability distributions, we performed stochastic simulation, randomly placing 10,000 pairs of landslides in the periods of two time slices and then recording the time passed between each pair. The median time passed between time slices $(t-t+n)$ was used as the time passed between time slices, and the first and third quartile were recorded to express uncertainty of the time between both time slices. This approach is unbiased and therefore no bias in consequent analyses is expected.

The third method quantifies the relation between area and number of overlaps. This method was used to quantify repeated overlapping over multiple time slices of the multi-temporal inventory. Every time slice of the inventory was rasterized at $1 \mathrm{~m}$ resolution and the cells involved in landsliding in each inventory were given a value of 1 . Then, all raster were summed, resulting in a raster where the values correspond to the number of overlaps over the entire multi temporal inventory. The 
analysis was performed using standard raster operations in ArcGIS. This measure does not change with the order of time slices, but is affected by the area involved in landsliding in the multi-temporal inventory, and by the number of time slices in it. Therefore, it was compared with the number of overlaps from a null model in which the observed area of landsliding per time slice was assigned randomly to the study area, regardless of slope or lithology. Since spatial information was not needed for this calculation, the null model was calculated in Microsoft Excel.

\subsubsection{Effect of different spatial association}

Spatial associations between landslides from a given time slice and the immediately preceding time slice were used (Figure 2.2d). Four classes of spatial association were defined (Figure 2.3). The 'inside' class contains landslides that are completely inside landslides from the earlier time slice. The 'partial' class contains landslides that partially overlap landslides from the earlier time slice. The 'touching' class contains landslides that are outside landslides from the earlier time slice, but that touch landslides from this time slice, and the 'outside' class contains landslides that neither overlap nor touch landslides from the earlier time slice. This classification was chosen based on the assumption that different spatial associations between landslides relate to different mechanisms in which landslides affect the probability of follow-up landslides. Landslides that are 'inside' earlier landslides, for instance, may particularly point to saturation of materials on top of an earlier landslide (Igwe and Fukuoka 2015), and landslides that are 'touching' an earlier landslide may point to a slope-effect because slopes are most strongly changed along the borders of earlier landslides (van Westen et al. 2006b). The 'outside' class was considered likely unrelated to earlier landslides, by any mechanism. 


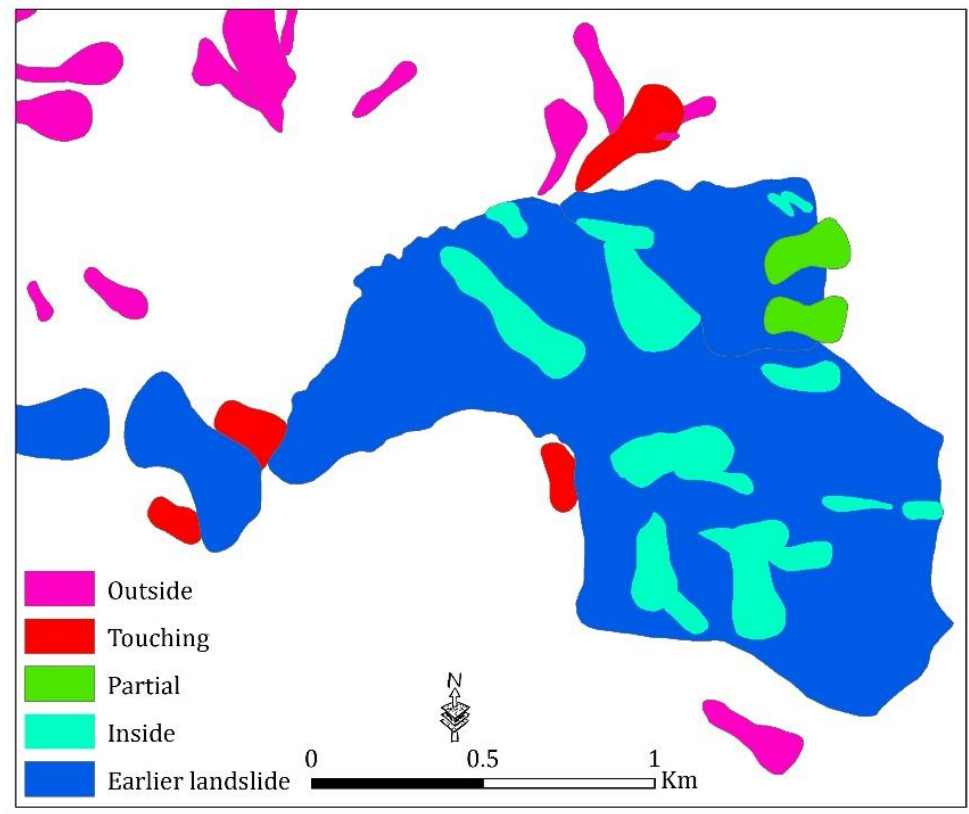

Figure 2.3. Examples of spatial association of landslides with landslides from the previous time slice.

Standard GIS tools were used to separate landslides from each time slice of the multi-temporal inventory, except the first time slice, into the four classes of association. The sub-classes of association (sub-inventories) were then merged over the different time slices. Then, the following geometrical properties were calculated for landslides in each merged sub-inventory: minimum, mean, and maximum area, roundness (a measure of shape), and the three parameters of the inverse-gamma distribution that is often used in landslide research to describe frequency-area relationships (Malamud et al. 2004), see below. This allowed us to test our second hypothesis: that landslides following up on earlier landslides have different geometric properties.

Minimum, mean, median and maximum sizes of landslides were calculated with standard GIS tools. Roundness (-) is introduced here as a simple measure of shape:

Roundness $=\frac{\text { theoretical circular perimeter }}{\text { actually measured perimeter }}$

Where the theoretical circular perimeter $(\mathrm{m})$ is the perimeter of the landslide, if it would have been perfectly round with the same area:

Theoretical circular perimeter $=2 \pi \sqrt{\left(\frac{A_{L}}{\pi}\right)}$ 
where AL is the area of the landslide $\left(\mathrm{m}^{2}\right)$. Roundness values closer to unity indicate round shapes, values close to zero indicate more elongated shapes (Figure 2.4). In earlier research, a landslide geometry generating algorithm was used to approximate the shape of landslides using geometric features such as upper and lower length and lateral boundary of the landslide scar (Chiang 2015). Taylor et al. (2015) approximated landslide shapes by ellipses and then used the length to width ratio of these ellipses to characterize shape. We argue that the proposed roundness is a simpler measure that makes no assumptions about landslide shape that nonetheless captures the most important difference between landslide shapes.
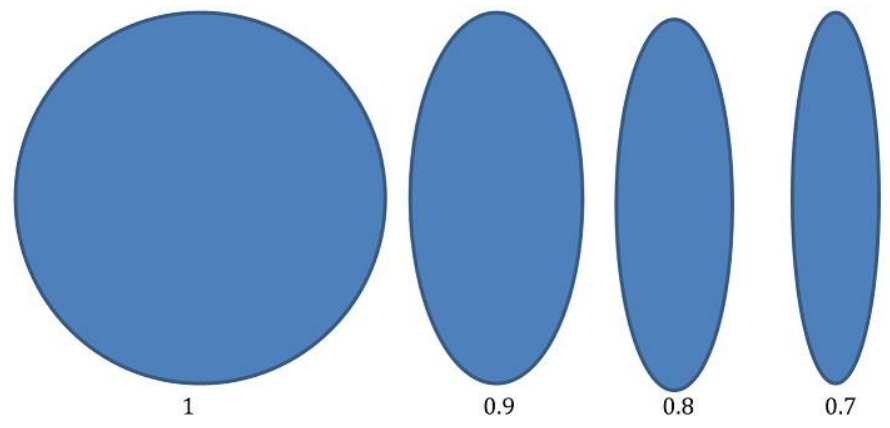

Figure 2.4. Example shapes illustrating different degrees of roundness.

To estimate power-law parameters, we fitted the three-parameter inverse Gamma distribution (Malamud et al. 2004) to the four sub-inventories, using LANDSTAT software (version 9) (Rossi and Malamud 2014). The three-parameter inverse gamma distribution is:

$\operatorname{pd}\left(A_{l} \mid \alpha, \eta, \lambda\right)=\left[\frac{\lambda^{2 \alpha}}{\Gamma(\alpha)}\right]\left[\left(\frac{1}{x+\eta^{2}}\right)^{(\alpha+1)}\right] \exp \left[-\frac{\lambda^{2}}{x+\eta^{2}}\right]$

Parameter $\alpha$ controls the steepness of the right tail of the probability density function. Parameter $\eta$ controls the steepness of the left tail of the probability density function. Parameter $\lambda$ controls the position of the rollover. From these parameters, the rollover, the most likely size of a landslide, can be calculated. Maximum likelihood estimation was used and uncertainties for the three parameters and the rollover were calculated using bootstrapping with 250 repetitions.

We used standard Analysis of Variance to test whether differences in landslide properties between sub-inventories are significant, complemented with T-tests for normally distributed properties (Student 1908) and U-tests for non-normally distributed properties (Mann and Whitney 1947). 


\subsection{Results}

\subsubsection{Degree of overlap}

Over time, significant differences occur between the theoretically unaffected area (Eq. 1), and the actually unaffected area (Eq. 2) (Figure 2.5). After the last time slice in the inventory, from May 2010, $7.8 \%$ of the area has not experienced landsliding during the entire period of observation because of overlapping landslides. Clearly, there is a significant amount of overlap between landslides.

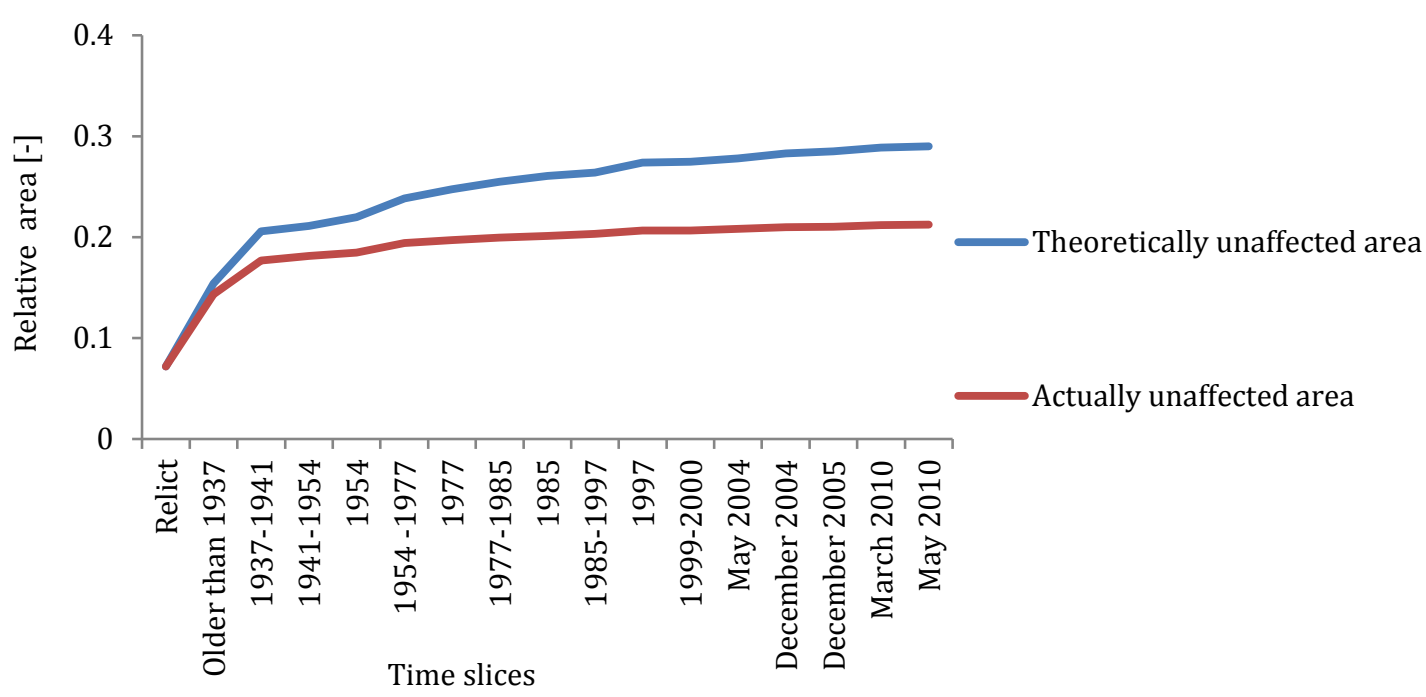

Figure 2.5. Theoretically and actually relative unaffected area by landslides in the Collazzone study area.

The overlap index (Eq. 3) appears to be negatively correlated with the time passed between time slices, although variation in overlap index is large, especially when observations are close together (Figure 2.6). The overlap of landslides with those from an earlier time slice appears to decrease substantially over a period of about 10 years. This suggests that the cause of new landslides is not completely external. If the cause of overlap was completely external (for instance through repeated landsliding at particularly dangerous locations), then no relation between overlap index and the amount of time passed between slides should exist.

Instead, earlier landslides themselves appear to affect the probability of reoccurrence to a substantial degree - probably through legacy effects. Importantly, this effect decreases over time. This means that if a landslide happened longer ago, it is less likely to be overlapped by a future landslide. However, this is not always the case: some time slices that are close together in time, have low overlap index. 
Apparently, not all landslides affect their environment such that there is a larger probability for future landslides.

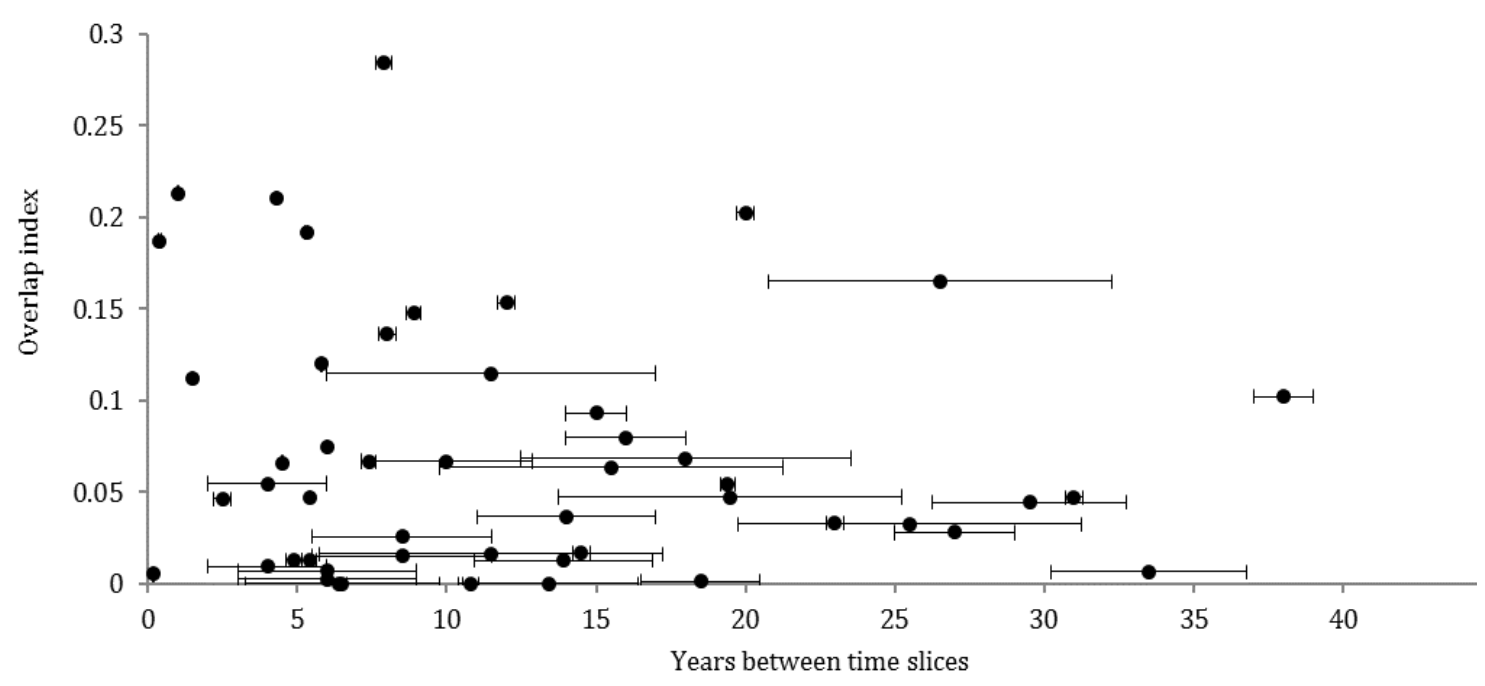

Figure 2.6. Overlap index as a function of time between time slices of the multi-temporal landslide inventory; the data shows a higher overlap index for time intervals up to approximately 10 years.

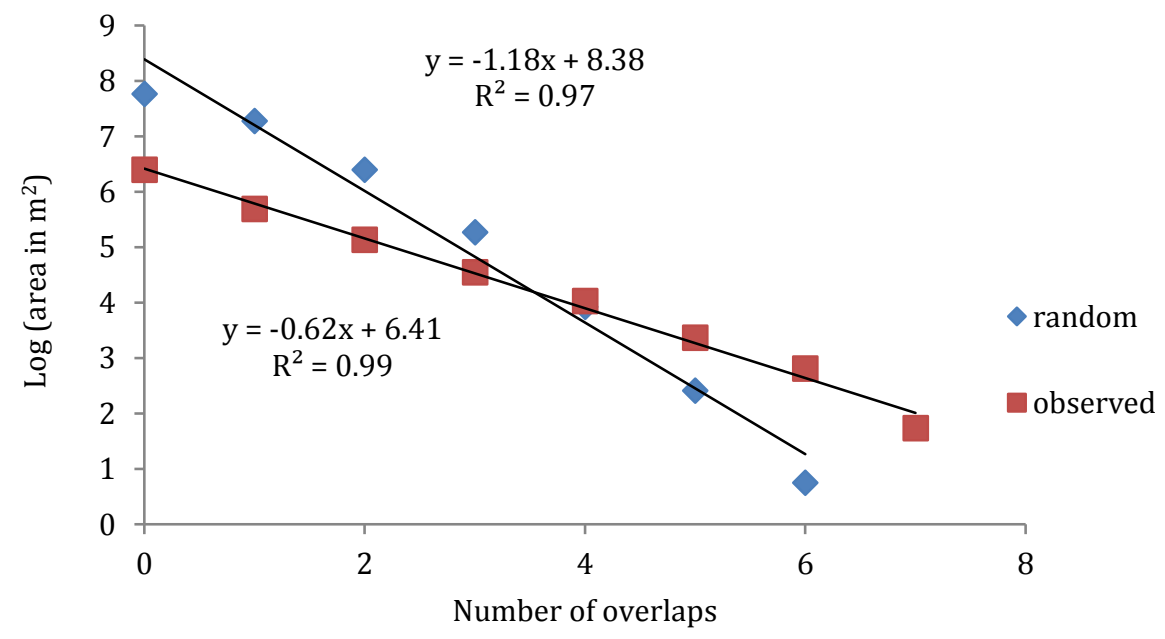

Figure 2.7. Area versus number of overlapping landslides. The area experiencing more than four overlaps is larger for the observed dataset than for the random dataset.

About $200 \mathrm{~m}^{2}$ of the study area has been affected seven times by landslides in the 17 time slices of the multi-temporal inventory (Figure 2.7). In the null-model in 
which landslide cells are placed randomly in the study area, seven overlaps do not occur. Overall, much more of the study area has been affected by three or more overlapping landslides than in the random model, whereas the area where no overlapping occurs, or where only one or two overlaps occur, is less than in the random model. The relation between number of overlaps and the logarithm of area is about twice as steep in the random model than in the dataset, again a clear sign of spatial overlap between follow-up landslides.

\subsubsection{Effect of spatial association}

Overall, $28 \%$ of landslides are in the classes inside (9\%), partial $(10 \%)$, and touching (9\%). The remaining $72 \%$ of landslides belong to the 'outside' class.

The mean size of the landslides with spatial association 'inside', 'partial' and 'touching' is larger than those with spatial association 'outside' (Table 2.2, $\mathrm{p}<$ 0.001). The subtle decrease in mean area from 'inside' to 'partial' and 'touching' is not statistically significant $(\mathrm{p}>0.05)$. 
Table 2.2. Geometrical and area-frequency distribution properties of landslides depending on spatial association.

\begin{tabular}{|c|c|c|c|c|c|}
\hline Inventories & & Inside & Partial & Touching & Outside \\
\hline & $\mathrm{n}$ & 165 & 199 & 185 & 2480 \\
\hline \multirow[t]{4}{*}{ Area (ha) } & Min & 0.03 & 0.01 & 0.02 & 0.002 \\
\hline & Mean \pm SD & $0.91 \pm 0.01$ & $0.89 \pm 0.01$ & $0.82 \pm 0.01$ & $0.51 \pm 0.01$ \\
\hline & Median & 0.38 & 0.46 & 0.46 & 0.21 \\
\hline & Max & 6.90 & 5.70 & 7.50 & 1.73 \\
\hline \multirow[t]{2}{*}{ Roundness } & Mean \pm SD & $0.79 \pm 0.10$ & $0.78 \pm 0.12$ & $0.78 \pm 0.12$ & $0.83 \pm 0.11$ \\
\hline & $\alpha \pm \mathrm{SD}$ & $1.04 \pm 0.08$ & $1.28 \pm 0.50$ & $1.78 \pm 0.25$ & $1.32 \pm 0.04$ \\
\hline \multirow{3}{*}{$\begin{array}{l}\text { Area-frequency } \\
\text { statistics }\end{array}$} & $\eta \pm S D$ & $16.2 \pm 4.3$ & $28.9 \pm 2.6$ & $32.8 \pm 4.2$ & $19.3 \pm 0.5$ \\
\hline & $\lambda \pm \mathrm{SD}$ & $52 \pm 6$ & $69 \pm 5$ & $90 \pm 11$ & $49 \pm 1$ \\
\hline & Rollover $\pm \mathrm{SD}\left(\mathrm{m}^{2}\right)$ & $1073 \pm 157$ & $1317 \pm 158$ & $1858 \pm 241$ & $670 \pm 25$ \\
\hline
\end{tabular}




\subsubsection{Shape factor}

Landslides that are not spatially associated with landslides from the immediately preceding time slice appear to be more round than other landslides (Figure 2.8). The type of spatial association is a significant determinant of shape (Table 2.3, $\mathrm{p}<$ 0.001 ). This is because of the higher roundness of the 'outside' type - all other types of association are not significantly different from each other $(\mathrm{p}>0.05)$.

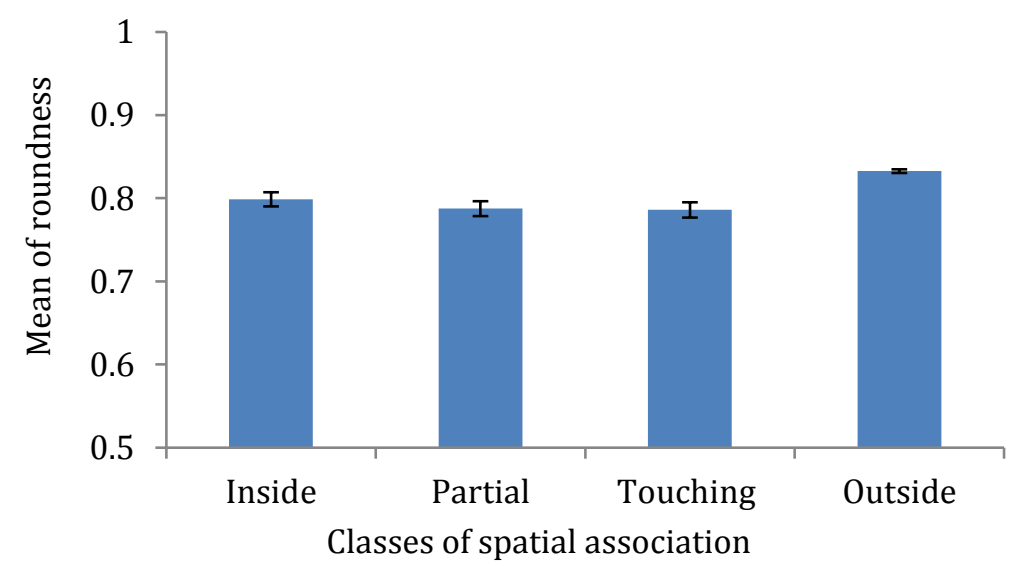

Figure 2.8. Roundness as a measure of shape for different classes of spatial association. Error bars indicate the standard error.

\subsubsection{Frequency-area statistics}

The frequency-area distributions for the four sub-inventories representing different spatial association exhibit power-law scaling for large and medium landslides with a rollover for small landslides (Figure 2.9a-d). The three parameters of the inverse gamma distribution, and the resulting rollover, differ between subinventories (Table 2.2). When the strength of spatial association between landslides decreases (from 'inside' to 'partial' and 'touching'), the exponent of the inverse power law $(\alpha)$ increases. The average value of $(\alpha)$ in the spatially associated sub-inventories is greater than in the 'outside' spatially un-associated subinventory. Interestingly, the value of the rollover is much smaller for the unassociated 'outside' sub-inventory than for the three other sub-inventories. The rollover also decreases with decreasing strength of spatial association. All differences between sub-inventories were significantly different $(p<0.001)$. 

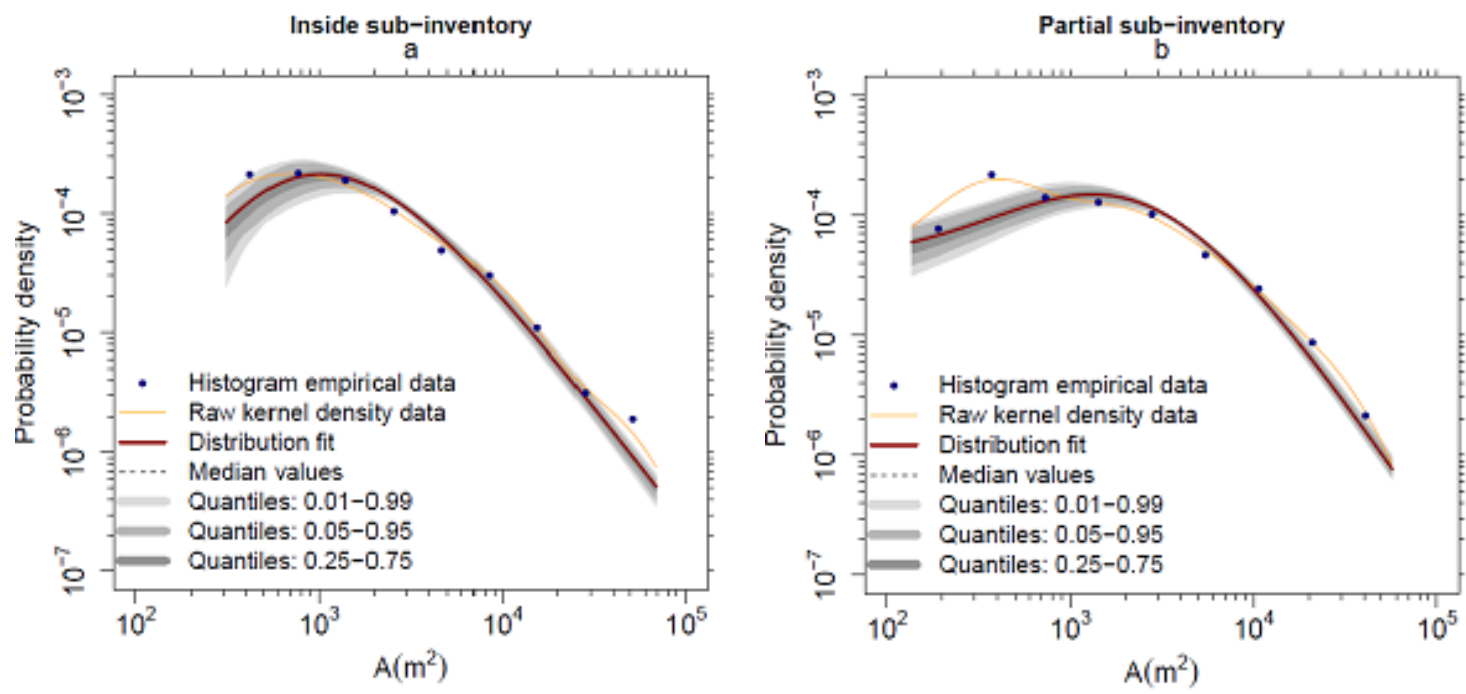

Touching sub-inventory

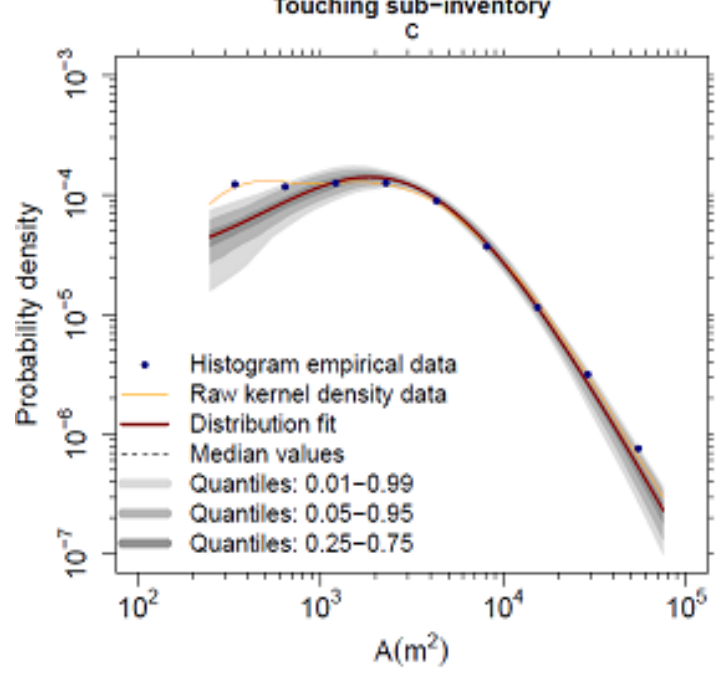

Outside sub-inventory d

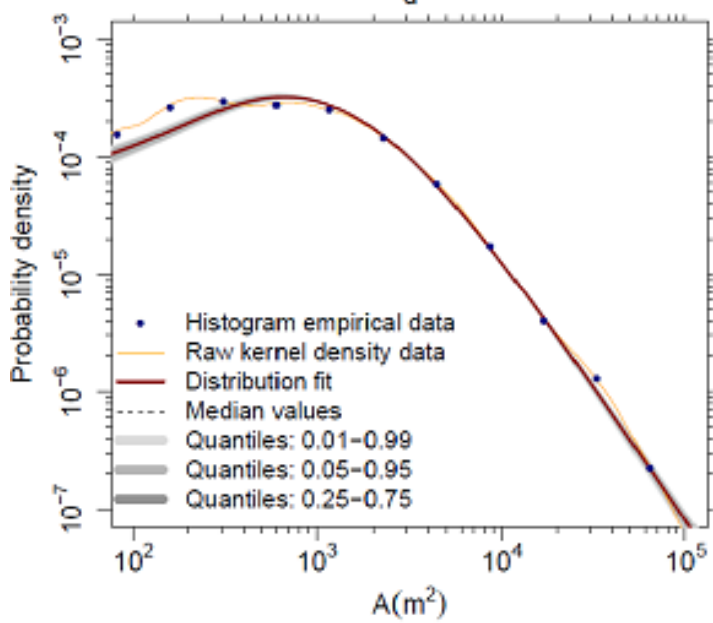

Figure 2.9. Comparison of the Inverse Gamma probability density function fit to frequency densities from four landslide sub-inventories representing different spatial association types. 


\subsection{Discussion}

In this discussion, we will first focus on our findings relating to the two hypotheses, before considering wider implications.

\subsubsection{Do landslides follow landslides?}

We found that the multi-temporal landslide inventory of our study site recorded substantial overlap between landslides, and an associated reduction in the fraction of the study area affected by landslides (Figure 2.5). The overlap is more for landslides happening sooner after an earlier landslide and decreases over a timescale of about ten years (Figure 2.6). This indicates that landslides in our study site do follow landslides. Importantly, it also suggests an internal control on the landslide system next to the range of intrinsic controls such as lithology, slope steepness and vegetation, which are commonly the focus of landslide susceptibility studies (Guzzetti et al. 2005; Van Westen et al. 1997). We propose that the mechanisms that can explain the nature of this internal control (i.e. a positive landslide-landslide effect that decreases over time) fit into two categories. First, the probability of landslides overlapping earlier landslides may decrease over time because the deposits of an earlier landslide stabilize due to e.g., a more stable slope geometry, the regrowth of vegetation, and the repair of soil structure and cohesion. Second, the probability can decrease over time because follow-up landslides have already occurred, erasing topographic instabilities that remained after an earlier landslide. Targeted field observations with high temporal resolution could quantify the relative importance of these two reasons by focussing on vegetation regrowth and detailed topographic evolution.

Regardless of the mechanism, our findings suggest that there is path dependency among landslides: older landslides act in some way or other as initiators for followup landslides for a certain period. This is also consistent with the number of landslide overlaps over the entire period captured by the multi-temporal inventory (Figure 2.7). This number of overlaps is larger than in the null model where landslides occur randomly in the study area - although when considered separately from Figure 2.6, this observation can also be explained by spatial differences in landslide susceptibility. Such landslide self-organization (Turcotte et al. 2002) into emergent patterns is not captured in traditional cause-effect studies of landslide susceptibility. It also adds an important consideration to the discussion about landslide self-organized critical behaviour (Guzzetti et al. 2002; Hergarten 2003; Turcotte et al. 2002). Apparently, not all landsliding potential ("metastable regions", Guzzetti et al., 2002, p171) is removed by a landslide - instead first landslides appear in some cases to increase the potential for follow-up landslides. The interplay between the traditional cause-effect approach and path dependency in a self-organization approach should be explored and quantified in follow-up studies. 
The period over which the positive landslide-landslide effect is observed - about ten years in our study area - is very likely specific for the local settings and other areas with similar climate, geology, topography, soils and vegetation. We maintain that this period is a measure of landslide path-dependency that reflects the rate of processes that reduce landslide susceptibility after a first landslide, or the rate at which follow-up landslides occur in our study site (see above). In areas with more frequent landsliding, or in sites with more rapid vegetation growth that restores stability, it may be shorter (all other factors being equal).

\subsubsection{Are follow-up landslides different?}

We also hypothesized that follow-up landslides have different properties in terms of size, shape, and frequency-area statistics. This is to some extent the case. Mainly, the mean size of landslides is lower for landslides that are not spatially associated with earlier landslides, than for those that are. Apparently, on average, follow-up landslides are larger (although there are also less of them, Table 2.2). In addition, our empirical data suggest that there may be a slight decrease of size with decreasing strength of spatial association between landslides (from 'inside' to 'partial' to 'touching' landslides).

The larger average size of follow-up landslides may be explained by conditions that have changed after occurrence of an earlier landslide, leading to increased landslide susceptibility. These changed conditions may relate to changes in surface morphology (Schuster and Highland 2003), sediment properties (Chen 2009), the vegetation (Singh et al. 2014), slope angle and land use (van Westen et al. 2006a). The shape of follow-up landslides differs from the shape of other landslides (Table 2.2). Landslides that are spatially associated with earlier landslides are less round (on average) than those that are not $(p<0.01)$. The difference in roundness can be explained by two mechanisms. First, weaker materials in an earlier landslide may move faster, and therefore further than other materials. This would then be reflected in less round shape. Second, especially for landslides of the 'touching' class, an earlier landslide may be higher in the landscape, deflecting landslides downslope, which leads to them being more elongated.

Both spatially associated landslides ('inside', 'partial' and 'touching' subinventories) and spatially un-associated landslides (outside sub-inventory) follow a power-law scaling in the right tail of inverse Gamma distribution for large and medium landslides with an exponential rollover in the left tail of distribution. However, the exponent of the inverse power-law $(\alpha)$ and rollover $(\lambda)$ differ between spatially associated landslides (even between sub-inventories) and spatially unassociated landslides (Figure 2.9a, b, c and d)). The exponent of inverse power-law $(\alpha)$ on average in spatially associated sub-inventories (i.e., 'inside', 'partial' and 'touching') are greater than un-associated sub inventory ('outside'). For landslides that are spatially associated with earlier landslides the exponent of power law $(\alpha)$ increases when the strength of the spatial association (from 'inside' to 'touching') decreases (Table 2.2). The exponent of the inverse power law $(\alpha)$ for 'touching' landslides $(\alpha=1.78)$ seems is consistent with the most reported range of values in 
the literature $(1.5<\alpha<2.5)$ (Borgomeo et al. 2014; Guzzetti et al. 2002; Malamud et al. 2004; Van Den Eeckhaut et al. 2007). The other landslides (i.e., 'inside', 'partial' and 'outside') exhibited lower values for exponent of the inverse power law ( $\alpha$ ) (i.e., $1<\alpha<1.5$ ) (Table 2.2). The small exponent of the power law in the sub-inventories suggests that larger landslides are contributing to each sub-inventory (Borgomeo et al. 2014; Van Den Eeckhaut et al. 2007).

The rollover $(\lambda)$, which represents the size of the most frequent landslide, is larger for spatially associated landslides than for non-spatially associated landslides ('outside', Table 2.2). Interestingly, the rollover for the 'touching' landslides is three times larger than for the 'outside' landslides, and larger than for the 'inside' and the 'partial' landslides. Analogously to our explanation for lower roundness, this may be explained by a boundary effect that can be caused by steeper slopes on the sides of an earlier landslide. The lateral boundary of a landslide is an intrinsically more disturbed part of a landslide, where the mechanical properties are weaker and infiltration is commonly larger. These factors contribute to instability, and hence to larger landslides.

\subsubsection{Implications for landslide susceptibility assessment}

The traditional division between landslide susceptibility and hazard is that the former describes spatial differences in landslide probability, and the latter describes the likelihood of landslide occurrence in time and space (Guzzetti et al. 1999; Varnes 1984). Our results suggest that a time-related internal control - the time that passed since an earlier landslide occurred - also plays a role in determining how susceptible a location is to landsliding. Apparently, susceptibility is not time-invariant, and contains a temporal element that traditionally was seen as part of the definition of hazard. Therefore, spatial and temporal probabilities of landsliding are not independent (Guzzetti et al. 2005). This means that the susceptibility of a location changes over time, as the effects of an earlier landslide slowly disappear. This can be expressed as:

susceptibility $_{s, t}=f\left(\right.$ conditioning attributes $_{s}$, previous landslides $\left._{s, t}\right)$

where the susceptibility for landsliding of a certain location $s$ at time $t$ is not only a function of conditioning attributes such as slope or lithology, but also of the time passed since an earlier landslide in the same location or close by. In our dataset, the function describing the effect of earlier landslides on susceptibility is positive and decreases over time (e.g., Figure 2.2). An earlier landslide appears to make a location more likely to experience landsliding again over a period of about ten years, after which susceptibility appears to return to its previous value (Figure 2.10). We explored possible mechanisms for this effect above. 


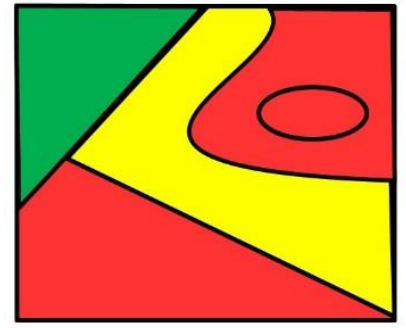

0

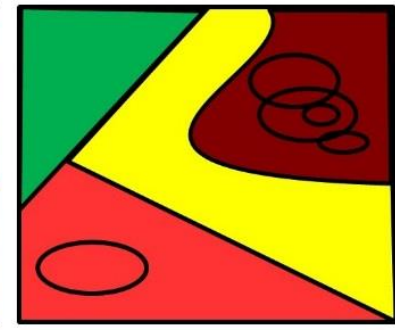

10

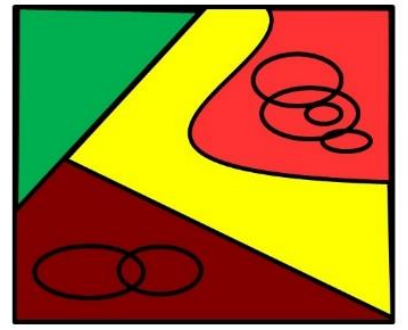

$30 \rightarrow$

Time (year)

Figure 2.10. A landslide susceptibility map bases on slope units (coloured polygons) with the occurrence of landslides over time (ellipses with black outline). In the susceptibility map, green means low risk, yellow means moderate risk, light red means high risk and dark read means very high risk. The implication of our work is seen in the clustering of landslides after a first landslide happens within a time scale of about 10 years. This is reflected in a temporarily higher susceptibility in the affected slopes. The two temporarily highly susceptible slopes (dark red polygons) experience different landslide dynamics as a result of different landslide histories.

The effect of the type of spatial association with (or the distance to) an earlier landslide is complex (Table 2.2). In places, a closer spatial association of a landslide with an earlier landslide leads to properties closer to spatially un-associated landslides (e.g. for the rollover), whereas in other places it leads to properties that are more different from un-associated landslides (e.g. the mean area) (Table 2.2). This complexity should be further researched since it may lead to a deeper understanding of the processes causing follow-up landsliding.

Other geomorphic settings could lead to different functions describing the effect of earlier landslides. For instance, exhaustion of sediment after landsliding, which occurs in locations where only little soil overlies bedrock (e.g., the steep basalt slopes of the Drakensberg in South-Africa (Singh et al. 2008) ), would lead to a decreasing of landslide susceptibility instead of an increase. Given that weathering is required for new soil to form, it would also probably be a longer term effect than the ten years that we observed (Figure 2.6). Exhaustion of soft material apparently plays no important role in our study site - fitting with its geology of predominantly soft, easily weatherable rocks. A complicating factor is that when the duration of the effect of landslides on landslide susceptibility increases much beyond a decade, other slow changes will also start affecting susceptibility, such as land use changes (e.g., deforestation, afforestation, changes in agricultural practices) and climate changes. This makes it more difficult to observe the effect that landslides themselves have on susceptibility.

The decreasing temporal effect of earlier landslides on susceptibility is to some extent comparable with the legacy effect of earthquakes on the occurrence of earthquake-induced landslides (Lin et al. 2007; Marc et al. 2015; Parker et al. 2015). For instance, Marc et al. (2015) demonstrated that landslide rates were 
Do landslides follow landslides?

significantly elevated within 0.7-4.5 years after four earthquakes with magnitude higher than 6 Richter. After this, rates dropped gradually back to pre-earthquake levels.

\subsubsection{Implications for mapping and monitoring of landslide populations}

Most landslide inventories are not multi-temporal, although they do often describe landslides that occurred over a longer period, or in response to several events. From such inventories, it is not possible to estimate the time-dependent effect of landslides on landslide susceptibility. In settings where that effect is strong, this may lead to susceptibility assessments that are biased. In statistical terms this would be because the landslide observations used to estimate a susceptibility model or map are not independent. In geomorphic terms, if the landslide-landslide effect is positive and decreases over time (Figure 2.6), resulting susceptibility maps would probably be biased to a smaller range of conditioning attributes than would be otherwise the case. Some places that are susceptible to landsliding would then possibly be considered safe. In case of a negative landslide-landslide effect, they would probably be biased to a larger range of conditioning attributes. In this case, some places that are safe would possibly be considered susceptible. How substantial these biases are, should be the subject of further study. Clearly, this calls for more multi-temporal landslide inventories.

\subsection{Conclusions}

In our study area, landslides follow landslides - and they do so more often than expected based on a random control. This landslide path-dependency is strongest over a timescale of about ten years, after which the effect of earlier landslides on follow-up landslides appears to decrease. This legacy effect has a considerable consequence on the relative area that is affected by landslides. Follow-up landslides differ from non-follow-up landsides in terms of size and shape: they are typically larger and less round. Also, the exponent of the power-law $(\alpha)$ and the value of rollover in spatially associated landslides on average are greater than in spatially un-associated landslides. From these findings we conclude that landslide susceptibility in our study area should be considered as a dynamic measure that reflects path dependency and self-organisation in landslides. Susceptibility changes because the effect of previous landslides on susceptibility, which in our case is positive, decreases over a period of about 10 years. Although our research concentrated on the Collazzone region in Italy, we expect these results to be relevant for other landslide prone areas as well.

Acknowledgements. J Samia acknowledges financial support from the Ministry of Sciences, Research and Technology of Iran. The comments and suggestions of two anonymous reviewers have greatly helped us in preparing this manuscript. We gratefully acknowledge this help. 


\section{Chapter 3}

\section{Characterization and quantification of path dependency in landslide susceptibility}

This chapter is based on:

Samia, J., Temme, A., Bregt, A., Wallinga, J., Guzzetti, F., Ardizonne, F., Rossi, M. (2017). Characterization and quantification of path dependency in landslide susceptibility. Geomorphology, 292, 16-24, DOI: 10.1016/j.geomorph.2017.04.039 


\begin{abstract}
Landslides cause major environmental damage, economic losses and casualties. Although susceptibility to landsliding is usually considered an exclusively locationspecific phenomenon, indications exist that landslide history co-determines susceptibility to future landslides. In this contribution, we quantified the role of landslide path dependency (the effect of landslides on landslides) using a multitemporal landslide inventory from Italy. The fraction of landslides following earlier landslides in the same location exhibited an exponential decay, with susceptibility increasing 15-fold right after an initial landslide, and returning to pre-landslide values after about 25 years. We investigated the role of the geometry and location of a previous landslide for the occurrence of follow-up landslides. Larger landslides are more likely to cause follow-up landslides. Also landslide shape, topographic wetness index, the vertical distance to the nearest channel network, the absolute profile curvature and relative slope position of an earlier landslide, however, are important in predicting whether a follow-up landslide occurs. Combined in a binary logistic model, these attributes correctly predict $60 \%$ of times whether a landslide will be followed-up. These findings open the way for time-variant mapping of susceptibility to landslides, by including the effect of the spatio-temporal history of landsliding on susceptibility.
\end{abstract}




\subsection{Introduction}

Landslides are recognized worldwide as a major natural disaster because of the destructive effects on society and the environment. In susceptible areas, rainfall, earthquakes, snowmelt (Malamud et al. 2004; Guzzetti et al. 2009), and anthropogenic activities (Meusburger and Alewell, 2008) are the main triggers for landslides.

Inventories of landslides document the distribution, type and extent of landslides (Guzzetti et al. 1996; Cardinali et al. 2001) and are a prerequisite for assessing susceptibility, hazard and risk assessment of landslides (Cardinali et al. 2002; Guzzetti et al. 2005; Van Westen et al. 2006). Multi-temporal inventories of landslides record the distribution of landslides in space and time and - although rare - are essential to increase understanding of evolutionary processes of landsliding (Guzzetti et al. 2012). Using such an inventory for the Collazzone region in Central Italy (see below), we recently demonstrated the existence of path dependency among landslides (Samia et al. 2017). The concept of path dependency originates from complex system theory and means that the current state of a system depends on the state of the system in the past (Phillips, 2006; Temme et al., 2015; Samia et al. 2017). Applied to landsliding, path dependency means that earlier landslides affect (the susceptibility for) future landslides. In this context, we defined a follow-up landslide as a new landslide that happens inside a previous landslide, or partly overlaps or touches it (Samia et al. 2017). This is different from a reactivation of the first landslide, in which case (almost) the entire previous landslide moves down. For the Collazzone study area, we found that this effect is positive (increased rather than decreased susceptibility after a previous landslide), and we found indications that the effect decreases over time (increased susceptibility appeared to be present only over timescales of decades after an earlier landslide) (Samia et al. 2017). Recently, such a legacy effect on the occurrence of landslide was also observed in the response to external drivers of landslides. For example, Parker et al. (2015) demonstrated the legacy effect of earthquakes on landslides in New Zealand. They showed that the Buller earthquake of 1929 triggered landslides immediately, and also reduced the stability of hillslopes, leading to the occurrence of more landslides in response to the 1968 Inangahua earthquake. Over shorter timescales, in rainfall-induced landslides, the legacy effect of antecedent rainfall, hydrological properties and accumulated damages in hillslopes facilitate the occurrence of landslides in later stages (Fan et al. 2015). Apparently, from our own work and from literature, path dependency has a role to play in explaining the future occurrence of landslides. The current operational approaches for mapping the susceptibility of landslides, however, do not take path dependency into account.

So far, mapping the susceptibility of landslides is commonly considered timeinvariant, and a function of spatially varying conditioning attributes. Maps of susceptibility are produced using a wide range of qualitative (Van Westen et al. 2003; Abella and Van Westen, 2008) and quantitative approaches (Van Westen, 2000; Guzzetti et al. 2005; Abella and Van Westen, 2008; Rossi et al. 2010). All these 
approaches study the relation between the distribution of landslides and conditioning attributes, such as topographic position, geological setting or land use. We proposed - in concept - a susceptibility approach that does take landslide path dependency into account (Samia et al. 2017). To the best of our knowledge, no previous research has quantified path dependency of landslides and has taken it into consideration in mapping the susceptibility of landslides. Nonetheless, clear candidate mechanisms can explain the path dependency of landslide : the effects of landslides on slope angle (Van Westen et al. 2006), surface morphology (Schuster and Highland, 2003), sediment properties (Chen, 2009) and vegetation (Singh et al. 2014).

To implement time-variant mapping of susceptibility, the path-dependent response of the landslide system needs to be quantified. To achieve this aim, we formulated four questions. First, how much is the susceptibility to landslides of an area increased after an earlier landslide? Second, how long does susceptibility to landslides remain elevated after an earlier landslide? Third, are landslides that cause follow-up landslides different from landslides that do not cause follow-up landslides? Answering this question affirmatively would mean that we are able to distinguish between landslides that cause follow-up landslides and those that do not. Therefore, the fourth research question is whether we can predict the followup occurrence of landslides as a function of the geometry and location of an earlier landslide. To answer these research questions, we again use the detailed multitemporal inventory of landslides from the Collazzone area in central Umbria, Italy (Guzzetti et al. 2006b; Ardizzone et al. 2013).

\subsection{Study area and data}

The Collazzone study area is located in the Umbria region in central Italy with an extent of $78.9 \mathrm{~km}^{2}$ (Figure 3.1). The elevation varies from 145 to $635 \mathrm{~m}$ above sea level with slopes extracted from a 10 by $10 \mathrm{~m}$ Digital Terrain Model (DTM) that range from 0 to $64^{\circ}$ (Guzzetti et al. 2006b). The area has been active in terms of occurrence of landslide from external triggers such as rainfall and rapid snowmelt (Guzzetti et al. 2006b). To avoid destruction by landslides, villages and individual farms in the area have been constructed on the top of ridges. A large part of the area is under arable land use and permanent forests are rare. A detailed multi-temporal inventory of landslides is available in which the size, type and extent of landslides in different periods were mapped and documented. Aerial photography, direct field mapping and high resolution stereo satellite images were used to prepare the multitemporal inventory of landslides. A full description of the study area and details about the multi-temporal landslide inventory have been published (Guzzetti et al. 2006a; Guzzetti et al. 2006b; Galli et al. 2008; Samia et al. 2017). For the present study, we have added two recent time slices to the multi-temporal inventory of landslides (landslide inventories mapped in 2013 using a stereo couple of GEOEYE images and in 2014 using a stereo couple of WorldView images), which now 
includes 17 time slices of mapped landslides from the period 1937-1941 to April 2014 (Figure 3.1).

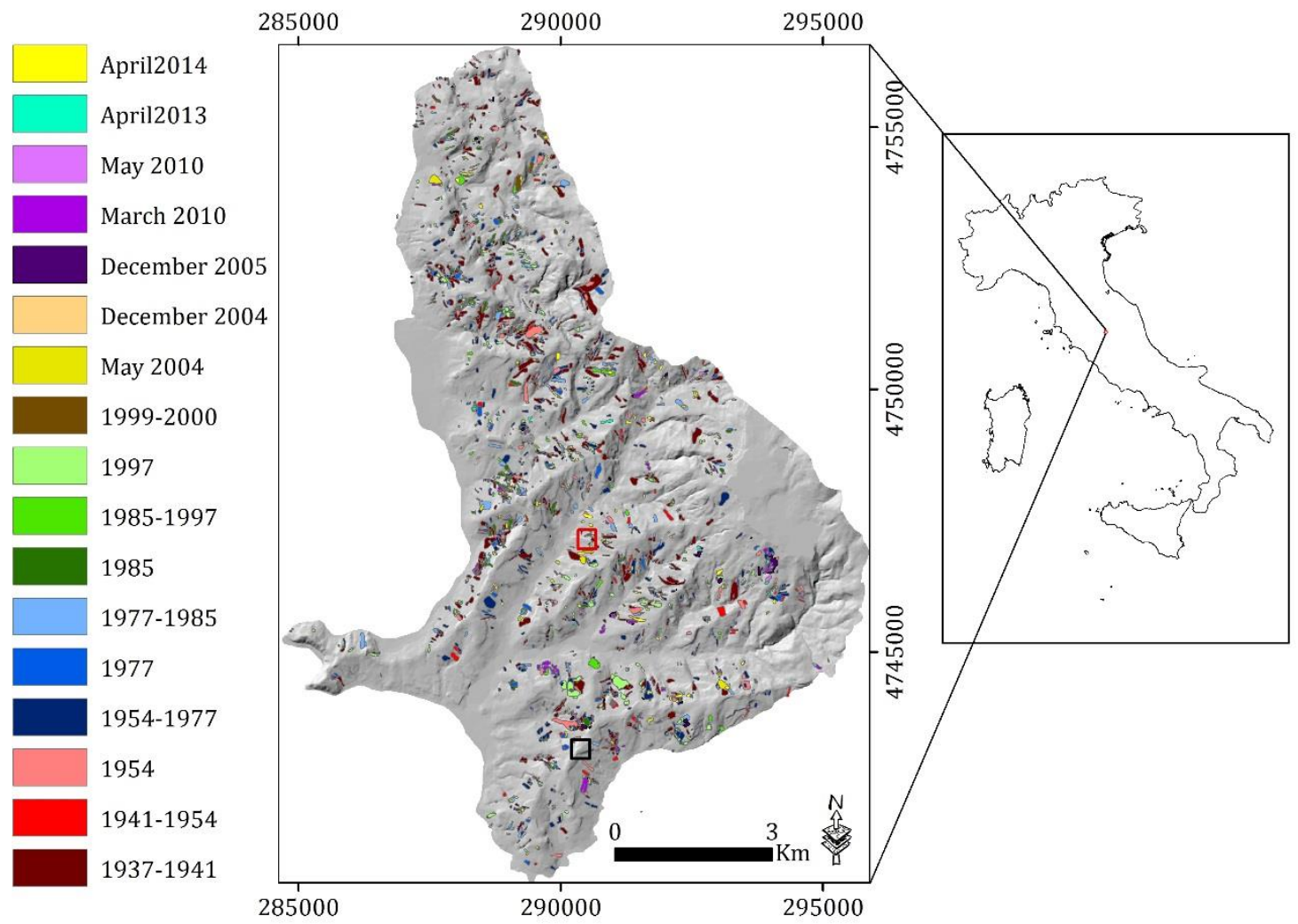

Figure 3.1. Location of the Umbria region and of the Collazzone study area (main map) (adapted from Samia et al. 2017). Multi-temporal landslide inventory including 17 time slices of landslide inventory overlying a shaded relief image (left map). The coordinate system of the relief image is EPSG:32633 (www.spatialreference.org). The red box indicates the location of Figure 3.7 and Figure 3.8.

Types of landslides in the study area, include: (i) slide (rotational and translational); (ii) flow; and (iii) slide-flow. Landslides range in age, type, morphology, and volume from very old, partly eroded, large and deep-seated slides to young and shallow slides and flows. Recent landslides are most abundant in the cultivated areas and are rare in the forested terrain, indicating a relationship with agricultural practices (Fiorucci et al. 2015). Information about geo-mechanical properties is limited and the available data revealed a significant variability in the mechanical and the hydrological properties of the geological materials present in the study area (Mergili et al. 2014). Santangelo et al. (2015) investigated the influence of bedding on abundance and types of landslides in the study area, using morpho-structural domains and multi-temporal landslide inventory maps. The analysis revealed that bedding conditions are important to predict the distribution and abundance of the relict, very old and the deep-seated landslides, but not the 
distribution and abundance of the shallow landslides that constitute the majority of landslides in the multi-temporal inventory.

\subsection{Methods}

\subsubsection{Temporal dynamics of landslide path dependency}

To answer the first two research questions about the strength and duration of path dependency, we investigated the number of follow-up landslides between time slices as a function of time between the time slices. This was complicated because some time slices describe multi-year periods, such as the time slice from 1937 to 1941, while some time slices describe the date of landslides relatively exactly (such as the December 2004 time slice) (Figure 3.1). For time slices describing periods, temporal midpoints were used to calculate the time difference with other time slices. For every time slice, the number of follow-up landslides from each of the following time slices was calculated. This was done using standard GIS functionality. In this context, follow-up landslides are defined based on the spatial association with earlier landslides; they could be either completely inside, partly overlap or touch landslides from landslides in an earlier time slice (Figure 3.2) (Samia et al. 2017). 


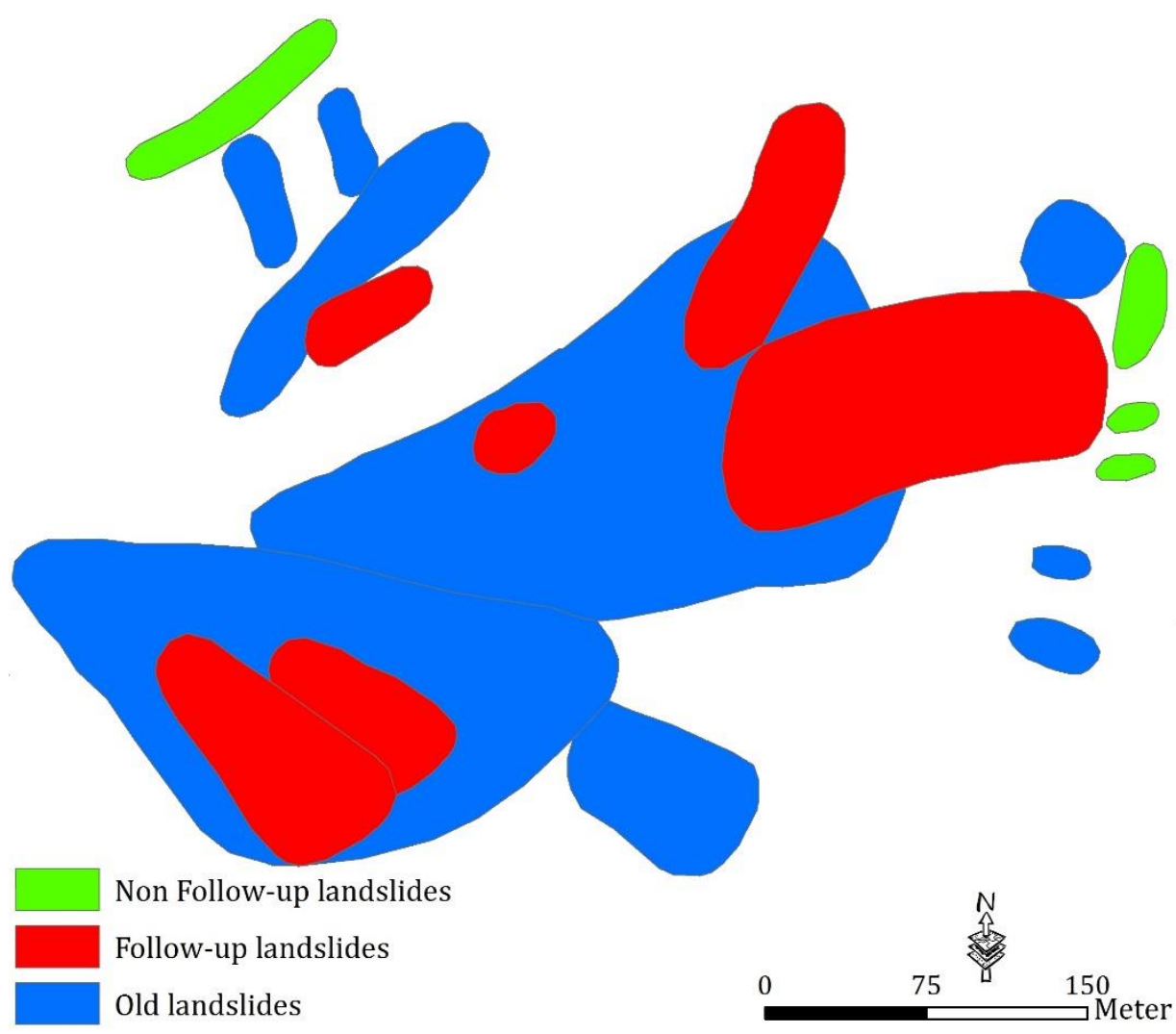

Figure 3.2. Examples of follow-up landslides and non-follow-up landslides with landslides from earlier time slice of multi-temporal inventory of landslides.

For example, from the earliest time slice with temporal midpoint in 1939 until the newest time slice in April 2014, we calculated the number of follow-up landslides for each of the 16 subsequent time slices, and documented the time passed between the initial and follow-up landslide. For the second time slice (temporal midpoint 1949), the approach was repeated, but now for only 15 subsequent time slices. This procedure was repeated until the two latest time slices (April 2013 and April 2014). Thus, in total, we calculated the number of follow-up landslides 127 times. Importantly, follow-up landslides are associated with the most recent earlier landslide. So, a landslide from 2014 that is spatially associated with a landslide from 1939 and with a landslide from 1954, was counted as a follow-up landslide from 1954 only. With this, we purely analyzed the effect of first landslides on second landslides. From this dataset, we calculated the follow-up landslide fraction $\rho(-)$ as:

$\rho=\frac{F L_{T 2}}{T L_{T 2}} \times \frac{A S}{A L_{T 1}}$ 
where $\mathrm{FL}_{\mathrm{T} 2}$ is the number of follow-up landslides in a younger time slice $\mathrm{T}_{2}, \mathrm{TL}_{\mathrm{T} 2}$ is the total number of landslides in younger time slice $\mathrm{T}_{2}$, AS $\left(\mathrm{m}^{2}\right)$ is the area of interest, and $\mathrm{AL}_{\mathrm{T} 1}\left(\mathrm{~m}^{2}\right)$ is the area affected by landslides in the older time slice $\mathrm{T}_{1}$.

We then related the 127 observations of follow-up landslide fraction ( $\rho$, Eq. 1$)$ to elapsed time between the time slices $\mathrm{T}_{1}$ and $\mathrm{T}_{2}$, and fitted an exponential function reflecting our earlier finding (Samia et al. 2017) that the follow-up landslide fraction from a previous landslide $\rho_{a}(-)$ decays over characteristic time scale b (y $\left.{ }^{1}\right)$, ultimately returning to its background value $\rho_{0}(-)$ :

$\rho(t)=\rho_{a} \exp ^{(b * t)}+\rho_{0}$

\subsubsection{Are landslides that cause follow-up landslides different from those that do not?}

To answer the third research question, we compared geometric and topographic attributes between landslides with follow-up landslides, and those without followup landslides. The analysis was carried out using analysis of variance (ANOVA). For the geometric attributes of the landslides, we used size and shape. For shape, we used the roundness (-) (Samia et al. 2017). In this measure, values closer to 1 indicate rounder shapes and values closer to zero indicate more elongated shapes (Figure 3.3).

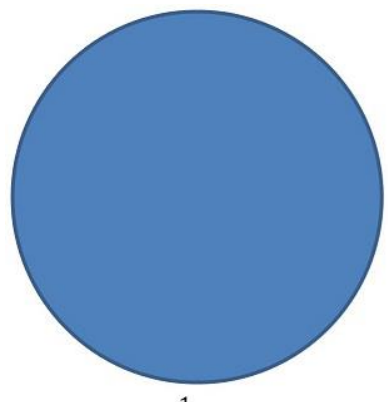

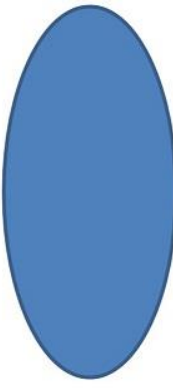

0.9
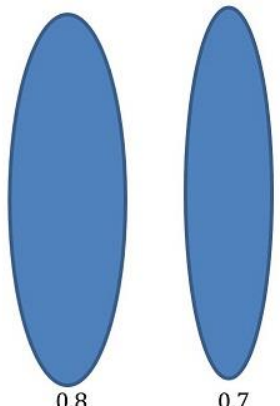

0.7

Figure 3.3. Different degree of roundness showing different shapes of landslides (Samia et al. 2017).

Topographic attributes were computed in the SAGA GIS (Conrad et al. 2015) exploiting a digital elevation model (DEM) with a $10 \times 10$ m ground resolution. Attributes selected were local terrain slope and aspect, Topographic Position Index (TPI), Topographic Wetness Index (TWI), plan and profile curvatures, absolute plan and profile curvatures, LS factor, vertical distance to channel network, and relative slope position. Aspect $\left({ }^{\circ}\right)$ was converted to northness $(-)$ and eastness $(-)$ using cosine and sine transformations (Harshburger et al. 2010). TPI $(\mathrm{m})$ is a measure that compares altitude of cells in a DEM with surrounding cells (Weiss, 2001). TWI $\left(\right.$ radians $\left.^{-1}\right)$, which is the logarithm of upstream area divided by slope (Moore and 
Grayson, 1991), indicates the importance of local topography on soil moisture. Plan curvature $\left(\mathrm{m}^{-1}\right)$ may affect the convergence or divergence of landslide deposits and flow in the landslide surface (Carson and Kirkby, 1972). Profile curvature $\left(\mathrm{m}^{-1}\right)$ controls the acceleration or deceleration of flow and may affect the movement of landslide deposits (Ohlmacher, 2007). We also calculated the absolute values of plan and profile curvatures to quantify deviation from straight slopes. The LS factor $(\mathrm{mm})$, which has been used in erosion studies, represents the slope length and steepness (Moore and Burch, 1986) and could be important in the process of landslide evolution. We used the vertical distance to channel network $(\mathrm{m})$ (Bock and Köthe, 2008) to possibly capture landslides happening very close to a channel that leave less potential for follow-up landslides. Relative slope position (-), the height of a location above the nearest downslope channel relative to the height of nearest upslope crest above the same location (Vaughn et al. 2015), offers a measure of remaining potential energy: landslides following those with very low relative slope position have limited remaining potential energy.

We then used one-way analysis of variance to evaluate whether any of the geometric and topographic attributes differ between earlier landslides where follow-up landslides occurred, and those that did not.

\subsubsection{Prediction of follow-up landslide occurrence}

Logistic regression - a multivariate statistical model that is widely used in the assessment of susceptibility to landslide (Cox, 1958; Lee and Sambath, 2006; Rossi et al. 2010; Rossi and Reichenbach, 2016) - was used to predict which landslides would experience a follow-up landslide. We applied logistic regression to predict the occurrence of follow-up landslides based on the geometric, topographic, and conditioning attributes of earlier landslides. We took care that no pair of these attributes had a correlation of more than 0.6 to avoid multicollinearity. In the logistic regression model, the occurrence or non-occurrence of follow-up landslides was considered as the binary dependent variable. We prepared different models using different sets of independent variables: only geometric variables, only topographic variables, only conditioning variables (land use and geology), and different combinations of these sets. We used 10 datasets of 598 landslides. Each dataset consisted of all 299 observed earlier landslides that do have follow-up landslides and 299 earlier landslides without follow-up landslides (randomly selected out of a total of 2,554 landslides without follow-up). For each dataset of 598 landslides, 70 percent was again randomly selected for calibration (training) and the remaining 30 percent was used for validation (testing). Model performances were evaluated with the Receiver Operating Characteristic (ROC) (Green and Swets, 1966; Mason and Graham, 2002; Beguería, 2006; Fawcett, 2006), in particular using the area under the ROC curve (AUC), a quantitative measure of model performance (Mason and Graham, 2002; Fawcett, 2006; Van Den Eeckhaut et al. 2009). Ranges of AUC values were used to quantify uncertainty and variation in the model. 


\subsection{Results}

\subsubsection{Temporal dynamics of landslide path dependency}

The follow-up landslide fraction ( $\rho$, Eq. 1 ) in the same location is high immediately after the first landslide, and then decreases gradually over time (Figure 3.4). The decline appears to be somewhat well described by an exponential decay function (Eq. 2) that decays to $25 \%$ of its initial value 10 years after the earlier (first) landslide. After 40 years, the value of $\rho$ is within $1 \%$ of its long-term value $\rho$ o. The value of the exponential coefficient $(b=-0.12 \pm 0.01 y$-1) shows that the increase in susceptibility for follow-up landslides decreases quickly after first landslide happened. The background susceptibility is provided by $\rho_{0}$, which is estimated at $2.85(-)$. The initial additional susceptibility because of a previous landslide $\rho_{\mathrm{a}}$ is estimated at $44(-)$, which implies that the susceptibility temporarily increases by a factor of 15 following a landslide. The residual standard error of the model is 12.67 $(-)$.

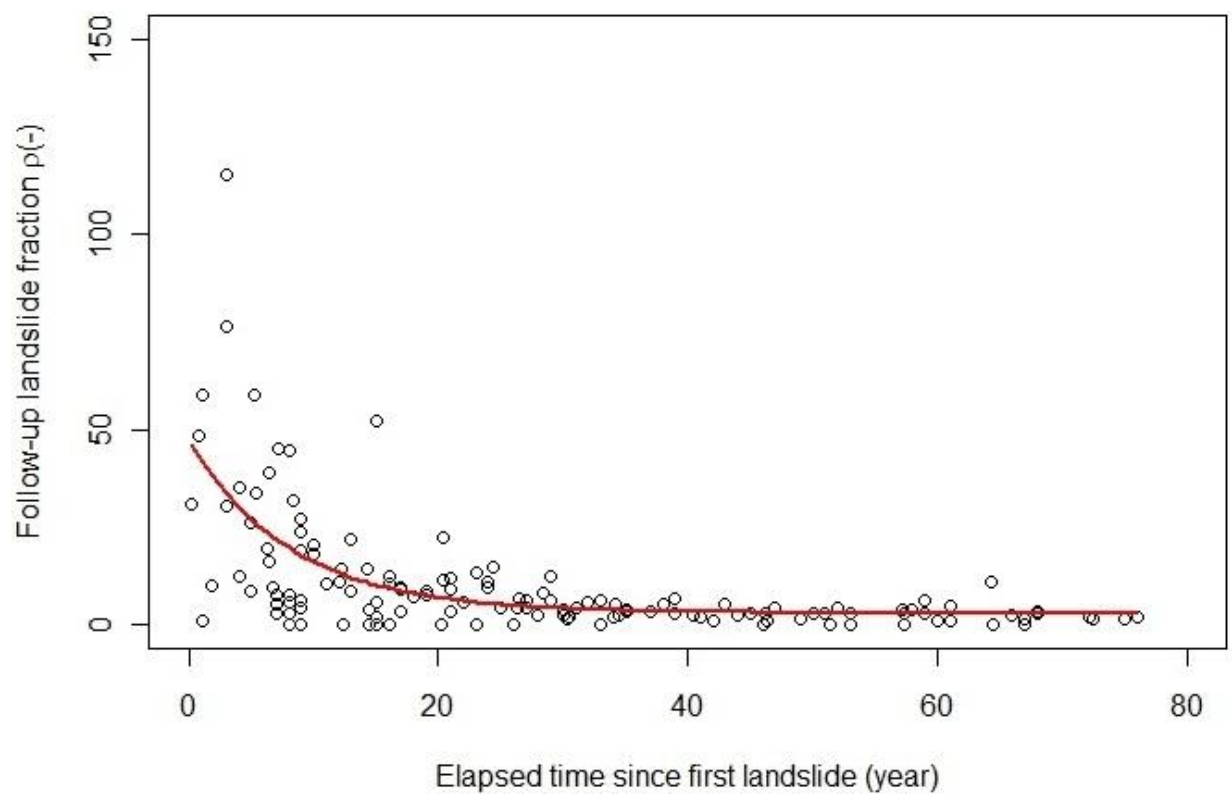

Figure 3.4. Temporal response of landslide path dependency with an exponential decay. 


\subsubsection{Which kind of geometric attributes and conditioning attributes of landslides are important for a landslide to cause a follow-up landslides?}

Here we investigate what types of geometric and topographic attributes of an earlier landslide are likely to induce a follow-up landslide. The histograms of geometric and topographic attributes of landslides that did and landslides that did not cause follow-up landslides demonstrate different distributions in some of these attributes (Figure 3.5). The mean area and roundness of landslides where followup landslides occurred are higher than those for landslides with no follow-up landslides $(\mathrm{p}<0.05$, Table 3.1). Therefore, larger and rounder earlier landslides are more likely to have follow-up landslides, in our study area.

The nature of the relation between the size of the landslides and the relative number of follow-up landslides (Figure 3.6) indicates that it is not merely a probability effect. In that case, a doubling in size would have led to a doubling in proportion of follow-up landslides.

Also, the mean TWI of landslides where follow-up landslides occurred is higher than the TWI of landslides without follow-up landslides $(\mathrm{p}<0.05$, Table 3.1). This indicates that landslides in wetter places are more likely to experience follow-up landslides. Moreover, the mean vertical distance to the channel network, the relative slope position and the absolute profile curvature of landslides that caused follow-up are lower than these attributes for landslides that did not $(\mathrm{p}<0.05)$. This reflects that landslides experiencing follow-up landslides typically occur in lower slope positions, and are closer to valley bottoms. Differences between both groups of landslides in terms of slope steepness, northness, eastness, plan curvature, profile curvature and LS factor are not statistically significant (all $p>0.05$, Table 3.1). Note that slope steepness apparently does not affect whether a follow-up landsliding occurs. This is interesting because slope is almost always an important conditioning attribute in mapping susceptibility. 

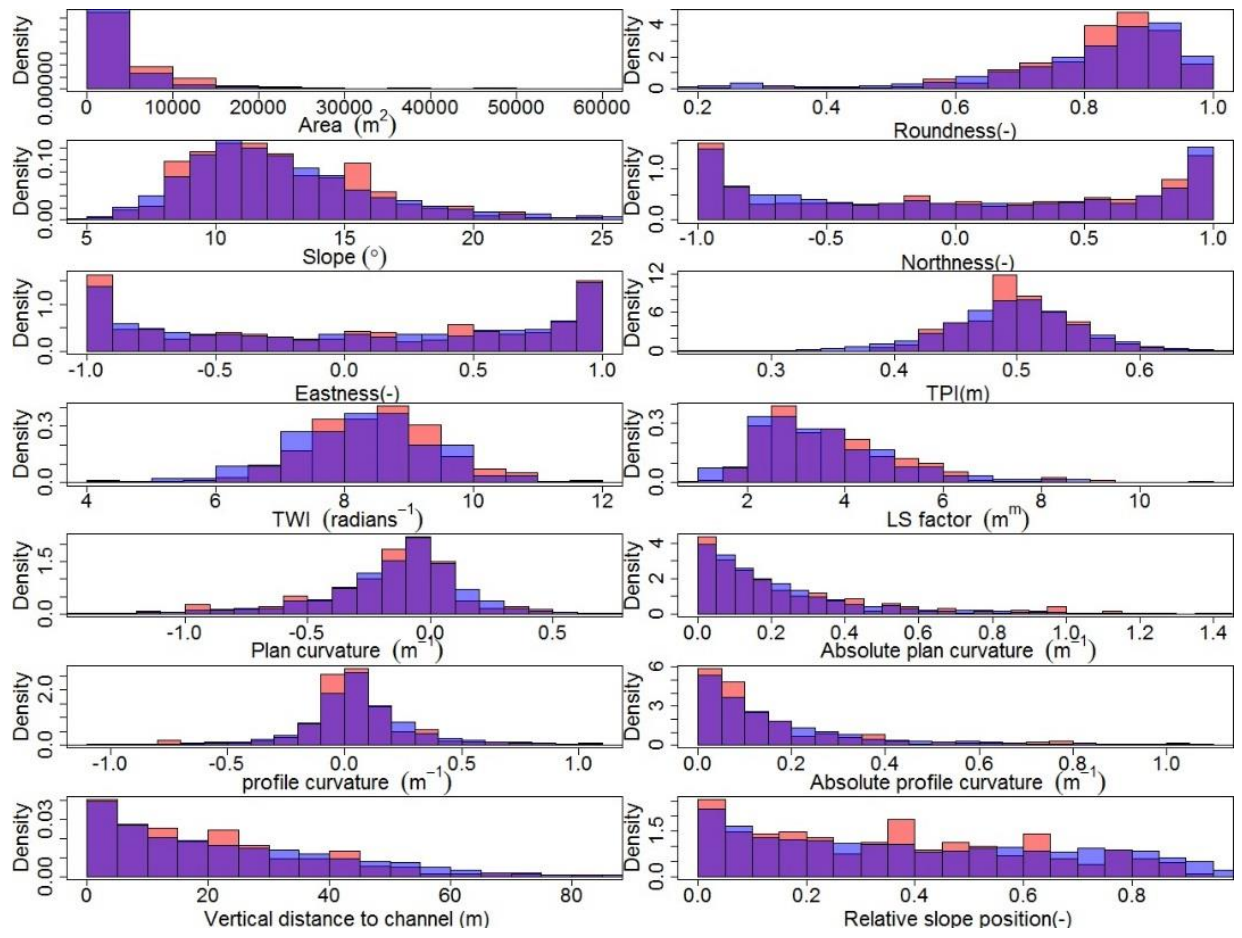

Figure 3.5. Histograms of geometric and topographic attributes of landslides with (red) and without (blue) follow-up landslides. The purple means overlap between red and blue.

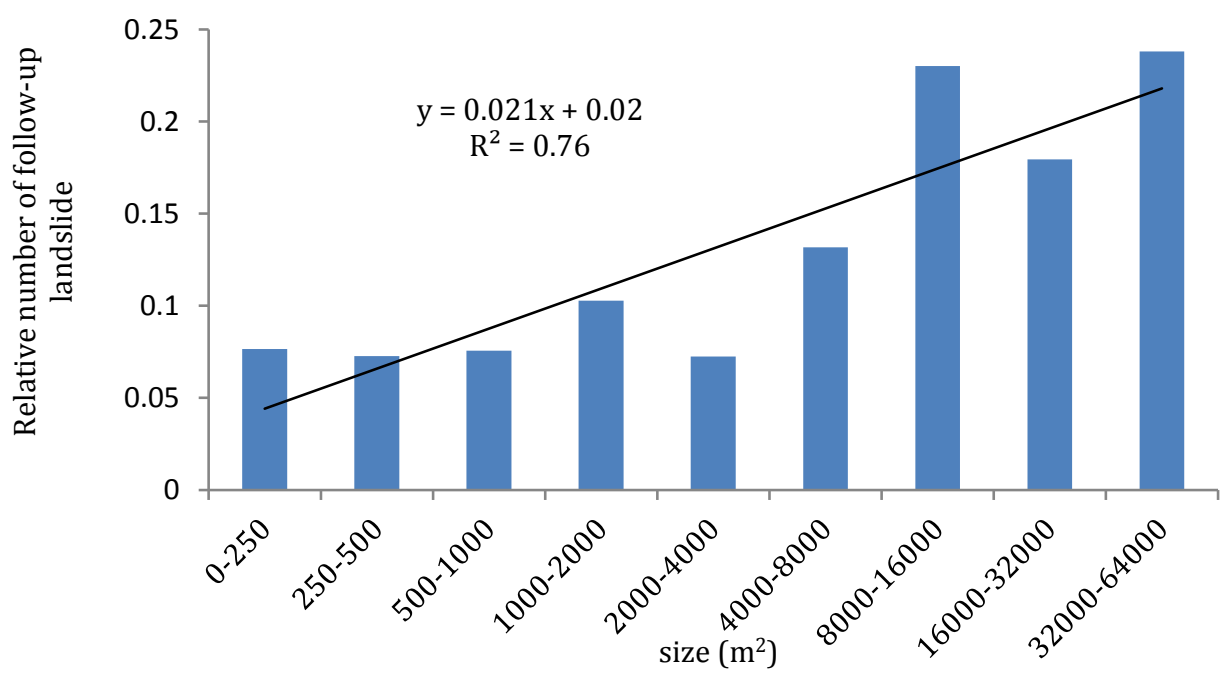

Figure 3.6. Relation between size of earlier landslides and relative number of follow-up landslides. 
Table 3.1. Results of one-way analysis of variance (ANOVA) for geometric and topographic attributes of landslides with follow-up (299 landslides) and without follow-up landslides (2594 landslides).

\begin{tabular}{|c|c|c|c|c|c|c|}
\hline $\begin{array}{l}\text { Geometric and topographic } \\
\text { attributes of landslides }\end{array}$ & Follow-up? & Mean & SD & Min & Max & Significance \\
\hline \multirow[t]{2}{*}{ size $\left(m^{2}\right)$} & Yes & 5518 & 7591 & 60 & 57081 & 0.001 \\
\hline & No & 3529 & 5472 & 23 & 75256 & \\
\hline \multirow[t]{2}{*}{ Roundness (-) } & Yes & 0.82 & 0.12 & 0.23 & 0.98 & 0.029 \\
\hline & No & 0.80 & 0.17 & 0.13 & 0.99 & \\
\hline \multirow[t]{2}{*}{ Slope $\left({ }^{\circ}\right)$} & Yes & 12.49 & 3.38 & 5.39 & 24.67 & 0.362 \\
\hline & No & 12.79 & 4.32 & 0.00 & 38.41 & \\
\hline \multirow[t]{2}{*}{ Northness (-) } & Yes & 0.009 & 0.711 & -0.99 & 0.99 & 0.860 \\
\hline & No & 0.017 & 0.706 & -0.99 & 0.99 & \\
\hline \multirow[t]{2}{*}{ Eastness (-) } & Yes & 0.013 & 0.704 & -0.99 & 0.99 & 0.608 \\
\hline & No & -0.008 & 0.707 & -0.99 & 1.00 & \\
\hline \multirow[t]{2}{*}{ TPI $(m)$} & Yes & 0.496 & 0.051 & 0.25 & 0.65 & 0.517 \\
\hline & No & 0.493 & 0.064 & 0.08 & 0.83 & \\
\hline \multirow[t]{2}{*}{ TWI (radians ${ }^{-1}$ ) } & Yes & 8.46 & 1.10 & 4.13 & 11.65 & 0.002 \\
\hline & No & 8.24 & 1.18 & 0.00 & 15.64 & \\
\hline \multirow[t]{2}{*}{ Plan curvature $\left(m^{-1}\right)$} & Yes & -0.179 & 0.300 & -1.39 & 0.66 & 0.208 \\
\hline & No & -0.150 & 0.379 & -5.89 & 2.57 & \\
\hline \multirow[t]{2}{*}{ Absolute plan curvature $\left(m^{1}\right)$} & Yes & 0.240 & 0.253 & 0.0001 & 1.39 & 0.851 \\
\hline & No & 0.244 & 0.327 & 0.0000 & 5.89 & \\
\hline \multirow[t]{2}{*}{ Profile curvature $\left(m^{-1}\right)$} & Yes & 0.038 & 0.258 & -1.00 & 1.07 & 0.220 \\
\hline & No & 0.071 & 0.452 & -4.14 & 5.75 & \\
\hline \multirow{2}{*}{$\begin{array}{l}\text { Absolute profile curvature }(\mathrm{m} \text { - } \\
\text { 1) }\end{array}$} & Yes & 0.166 & 0.202 & 0.0005 & 1.07 & 0.003 \\
\hline & No & 0.235 & 0.392 & 0.0000 & 5.75 & \\
\hline \multirow[t]{2}{*}{ LS factor $\left(m^{m}\right)$} & Yes & 3.73 & 1.46 & 1.06 & 11.47 & 0.146 \\
\hline & No & 3.60 & 1.51 & 0.00 & 16.32 & \\
\hline \multirow{2}{*}{$\begin{array}{l}\text { Vertical distance to channel } \\
\text { network }(m)\end{array}$} & Yes & 20.32 & 16.68 & 0.00 & 81.66 & 0.019 \\
\hline & No & 23.05 & 19.29 & 0.00 & 115.22 & \\
\hline \multirow[t]{2}{*}{ Relative slope position (-) } & Yes & 0.35 & 0.25 & 0.00 & 0.90 & 0.019 \\
\hline & No & 0.39 & 0.28 & 0.00 & 0.99 & \\
\hline
\end{tabular}




\subsubsection{Prediction of the occurrence of follow-up landslides}

The logistic regression model that combines geometric and topographic landslide attributes is best able to predict the occurrence of follow-up landslides, with AUC = $0.64 \pm 0.02$ for the 10 training data sets (calibration) and AUC $=0.58 \pm 0.04$ for the 10 validation data sets (Table 3.2 ).

Interestingly, the logistic regression model constructed using only the geometric landslide attributes of earlier landslides (inherent properties, i.e., size and shape) predicts follow-up landslides with an AUC $=0.60 \pm 0.01$ for the training dataset, which is slightly more than the model constructed with only topographic attributes $(A U C=0.57 \pm 0.03)$. The models that considered geology and land use were never significantly related to whether a landslide experienced follow-up sliding. Hence, no regression models that have them as explanatory variables, are reported. Apparently, in our study area geology and land use do not play an important role in determining whether a landslide will have a follow-up landslide. 
Table 3.2. Logistic regression models implemented to predict occurrence of follow-up landslides based on geometric and topographic attributes of earlier landslides. Numbers between parentheses indicate the number of times that variables were selected by logistic regression in 10 times repetition.

\begin{tabular}{|c|c|c|c|c|}
\hline $\begin{array}{l}\text { Set of } \\
\text { variables }\end{array}$ & $\begin{array}{l}\text { Variables available for } \\
\text { logistic regression }\end{array}$ & $\begin{array}{l}\text { Variables selected by logistic } \\
\text { regression }\end{array}$ & AUC calibration & AUC validation \\
\hline Geometry & 2 & Area (10), roundness (10), & $0.60 \pm 0.01$ & $0.55 \pm 0.03$ \\
\hline Topography & 12 & $\begin{array}{l}\text { Absolute profile curvature (6), } \\
\text { TWI (5), LS factor (4), slope } \\
\text { (4), relative slope position (2), } \\
\text { vertical distance to channel } \\
\text { network (1) }\end{array}$ & $0.57 \pm 0.03$ & $0.56 \pm 0.05$ \\
\hline $\begin{array}{l}\text { Geometry + } \\
\text { topography }\end{array}$ & 14 & $\begin{array}{l}\text { Area (10), Roundness (10), } \\
\text { absolute plan curvature (5), } \\
\text { absolute profile curvature (4), } \\
\text { LS factor (4), TWI (4), slope } \\
\text { (4), Vertical distance to } \\
\text { channel network (2), relative } \\
\text { slope position (2), eastness (1) }\end{array}$ & $0.64 \pm 0.02$ & $0.58 \pm 0.04$ \\
\hline
\end{tabular}




\subsection{Discussion}

We will first focus on the answers to our four research questions and then discuss the implications of our findings.

\subsubsection{Temporal dynamic of landslide path dependency}

The quantification of the timescales involved in landslide path dependency indicates that, in our study area, an increased susceptibility caused by earlier landslides is reduced to about a quarter of its magnitude after ten years, and is insignificant after $\sim 25$ years (Figure 3.4). This corroborates and refines our earlier findings where we found a positive landslide-landslide effect for a period of several decades (Samia et al. 2017). Additionally, we find that susceptibility immediately after a landslide is about 15 times higher than background levels.

Although it is beyond the scope of this study to identify the mechanisms that drive these temporal dynamics, we can make some inferences. This is based on the findings reported above and a field exploration of landslides in the study area, which we carried out after a rainstorm in May 2016. During the latter, we observed indications that the potential mechanism that causes landslides to increase susceptibility, results from changes of slope hydrology. Small ponds formed on and in landslide deposits, saturating the material on the surface. In several places, this seemed to induce repeated failures (Figure 3.7). Closer inspection revealed the existence of smeared surfaces on the sliding planes, which may limit infiltration through them, and, hence, cause saturation of the landslide deposit and areas further downslope (Figure 3.). Assuming that increased susceptibility indeed results from disruptions in slope hydrology, we propose that the decay of additional susceptibility after a landslide is caused by the slow restoration of the original slope hydrology and removal of smeared surfaces, most likely by means of root growth, soil faunal activity and ploughing (which reaches depths of typically $20 \mathrm{~cm}$ in the study area). 


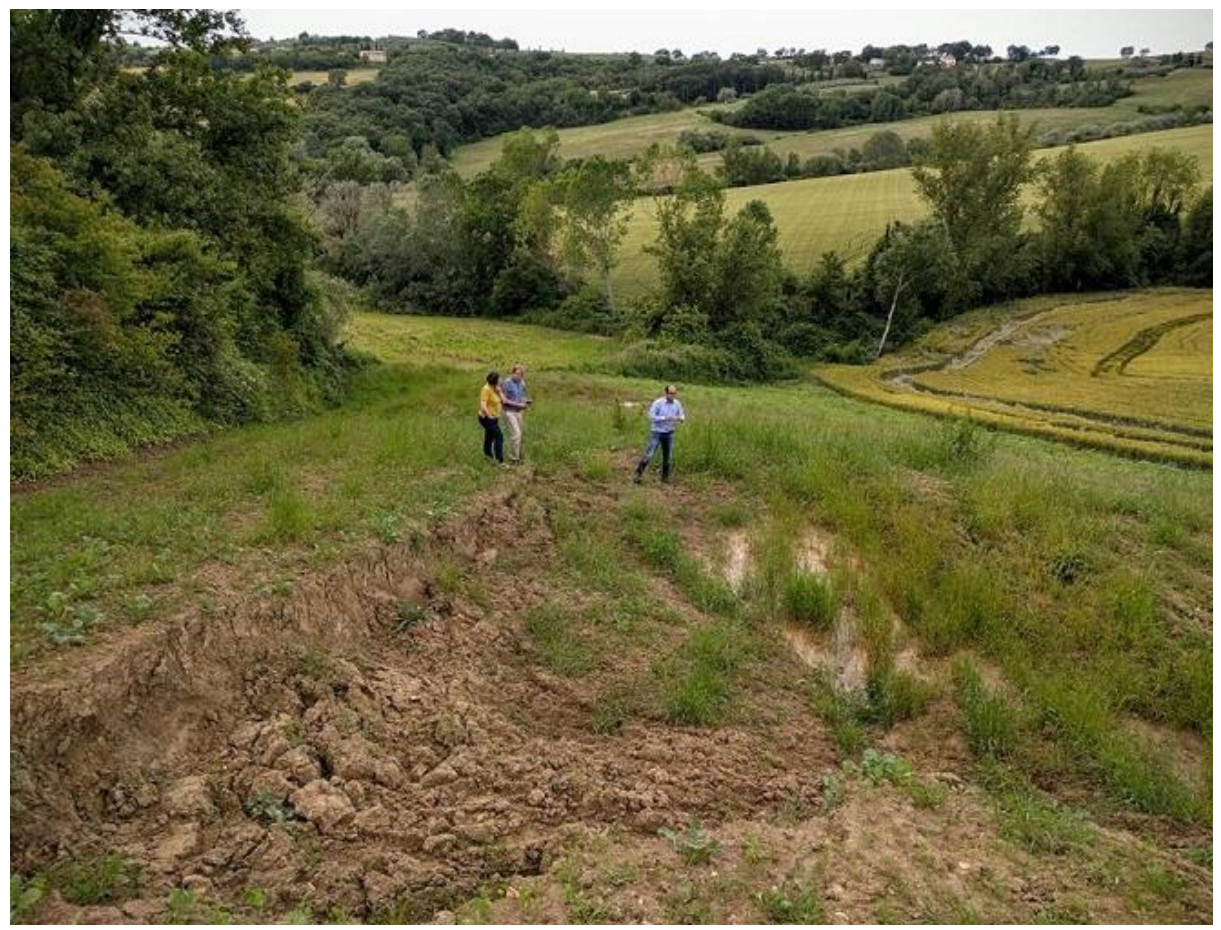

Figure 3.7. Ponding on top of landslide deposit (23-03-2016, Collazzone study area in Italy).

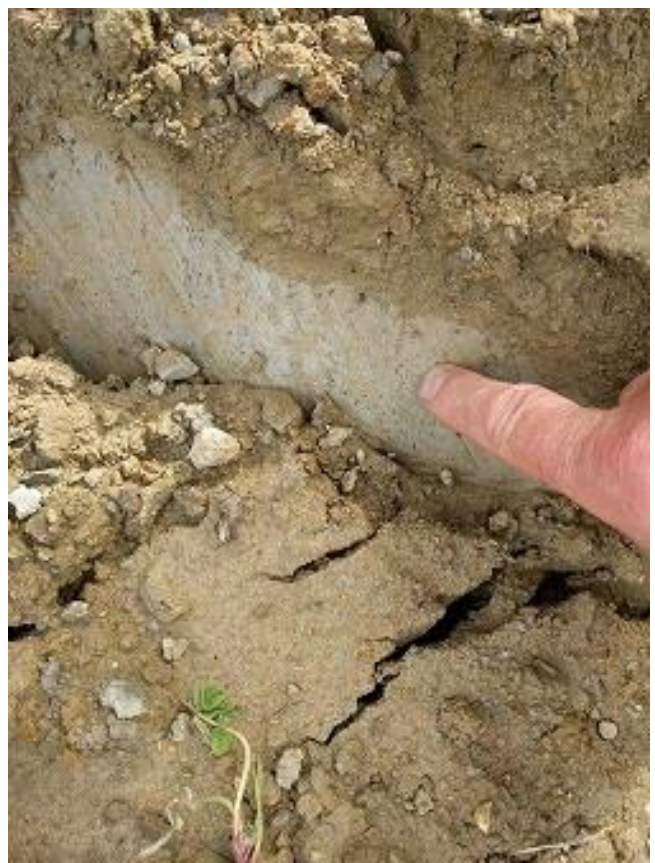

Figure 3.8. Smeared surface on landslide shear plane (23-03-2016, Collazzone study area in Italy). 
In some ways, the increased likelihood of follow-up landslides soon after an earlier landslide is similar to the increased occurrence of landslides after earthquakes. It has been suggested that this increase results from the (transient) reduction of ground strength (Marc et al. 2015) - a different mechanism than we propose for our observations. Marc et al. (2015) suggested that the intensity of landsliding could remain elevated for several months to years before ground strength is restored - about an order of magnitude smaller than the timescales we describe for the decay of increased susceptibility. Also, post-earthquake landslides and sediment delivery ratio after the 1999 Mw 7.6 Chi-Chi earthquake and Typhoon Toraji in Taiwan (Dadson et al. 2004) was elevated for only four years after the earthquake.

The slower decay of increased susceptibility that we observe, is likely a function of a different follow-up mechanism (subsoil hydrology disruption in our case versus ground strength reduction in both mentioned cases). These differences may reflect geological setting and disruptive agent (previous landslides in our case, versus earthquakes in both other mentioned cases). The fundamental difference from the perspective of mapping susceptibility, however, is that increased sliding after earthquakes happens in the same manner across large areas, whereas we describe the influence of individual landslides on sliding susceptibility in the immediate vicinity.

\subsubsection{Differences between landslides that experience and do not experience follow-up landslides}

We found that size and shape of a landslide play important roles in determining whether a landslide is likely to cause a follow-up landslide (Table 3.1, Figure 3.6). Earlier landslides that are larger and rounder lead to occurrence of more follow-up landslides. The role of size is easily explained: larger landslides result in more disruption of hillslopes, in terms of volume of material affected, and in terms of slope hydrology. The effect of size does not manifest itself through simply an increased probability of a new landslide happening close to or overlapping with, an older landslide - that would have led to a much stronger effect of size on the ratio of landslides that are follow up (Figure 3.6). Regarding the effect of shape, we suggest that rounder landslides are more likely to be slump-like (as opposed to more flow-like) and can, hence, result in stronger smearing of the subsurface.

The effects of these geometric landslide attributes fit with the concept of "landsliding potential" remaining after landslides occur (Samia et al. 2017). This landsliding potential was earlier called "meta stable regions" - the regions for propagation of landslides after they happened - to explain the concept of selforganized critical behaviour (Guzzetti et al. 2002). Apparently, some landslides create such "meta stable regions" better or more than others.

We also found that larger TWI, smaller vertical distance to channel network and lower relative slope position have a slightly positive effects on the likelihood of follow-up landsliding. The larger values of TWI in earlier landslides that 
experienced follow-up landslides may indicate that slides of wetter material are more likely to smear or otherwise hydrologically affect the subsurface. This finding at the aggregate level again suggests that slope hydrological changes made by a first landslide increase the likelihood of occurrence of a follow-up landslide. It is an open question whether earlier landslides in drier locations - with lower TWI values - do not create the kind of smeared surfaces that we observed in the field (Figure 3.), or that those locations are simply less likely to experience repeated levels of wetness that together with a smeared surface would lead to a follow-up landslide. Careful field observations of smearing and other subsurface changes caused by landslides along a slope transect from high to low TWI could shed more light on this question.

Apparently, slope steepness itself does not play a role in determining whether follow-up landslides occur (Table 3.1) - whereas it is often a prominent factor in determining susceptibility in the first place. Slope apparently controls susceptibility, but not how susceptibility changes locally after a landslide.

The results of ANOVA add an important point to the susceptibility of landslides where size of landslide needs to be considered. For prediction of follow-up landslides, differences in mean value of geometric attributes and topographic attributes of landslides are important. The mean values of roundness, TWI, vertical distance to channel and relative slope position are almost the same (Table 3.1). Therefore, they are useless for prediction whereas a clear difference between the mean value of size (area) should be considered as diagnostic criterion in landslide susceptibility. This was confirmed by the results of logistic regression model where the size of landslides predicted the occurrence of follow-up landslides (AUC $=0.60$ \pm 0.01 ) (Table 3.2). This stresses the importance of using inherent properties of landslides (in particular size of landslides) for the assessment of susceptibility to landslides.

A combination of geometric attributes and topographic attributes of landslide used in logistic regression increased the capability of prediction of occurrence of followup landslides (AUC $=0.64 \pm 0.02$ ) (Table 3.2). Apparently, geometric attributes of landslides help to predict the occurrence of follow-up landslides. Interestingly, adding geology and land use to the regression models did not improve predictions. This suggests that landslides have the potential to cause follow-up landslides regardless of the land use or geology. It must be mentioned, however, that the landslides in our study area occur in geology that almost uniformly weathers into clayey soils. Therefore, a possible effect of other geological settings on the likelihood of follow-up landslide remains to be investigated. Specifically, it is plausible that the main presumed follow-up mechanism in our study area - changes in slope hydrology caused by a previous landslide - is less important in areas with less clayey soils. 


\subsubsection{Role of landslide path dependency in evolutionary process of landslides}

Our results suggest that temporal landslide path dependency plays an important role in the long-term evolutionary process of landslides. Per our findings, where a certain type landslide affects a slope strongly enough, follow-up landslides are likely to continue to occur and eventually, clusters of landslides may form. Such clustering of landslides was mentioned earlier by Larsen and Montgomery (2012), who found spatial clustering of landslides in areas with higher rates of uplift. These authors do not refer to path dependency effects, however, and refer to landslides that are close to each other, but not directly spatially associated (as in Figure 3.2). To facilitate further discussion of both types of clustering, we propose to distinguish both types of clustering into conjunctive clustering, where landslides touch or overlap, and disjunctive clustering, where a group of landslides is closer to each other than to other landslides or groups of landslides. In our study area, path dependency between landslides contributed to the development of 444 direct clusters of landslides, containing 2,350 landslides $(77 \%$ of the total number of landslides). The largest cluster consisted of 55 landslides, which were recorded in 12 time slices. 738 landslides did not (yet) experience follow-up sliding (Figure 3.9).

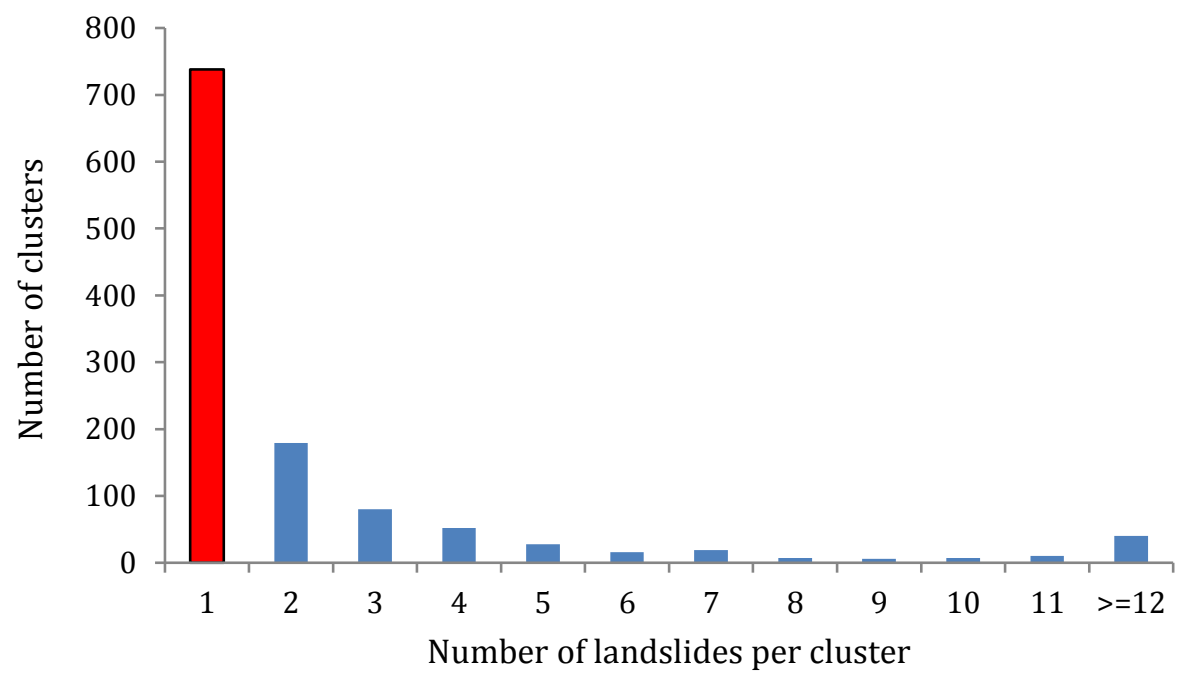

Figure 3.9. The number of clusters (landslide areas containing one or more (partially) overlapping landslides), shown as a function of the number of landslides per cluster recorded in the multi-temporal landslide inventory.

Following from the positive effect of landslide size on follow-up landslides, the size of an earlier landslide also has a positive influence on the total number of follow-up landslides in a cluster, and, hence, on the size of the clusters (Figure 3.10). This leads to the idea that, over longer timescales, a slope can be 'turned on' by one (large) initial landslide. The exponential growth response in role of size of first landslide on the number of landslides per cluster appears to describe this process 
adequately, although in reality it would be limited because landslides cannot be larger than the slopes they occur in.

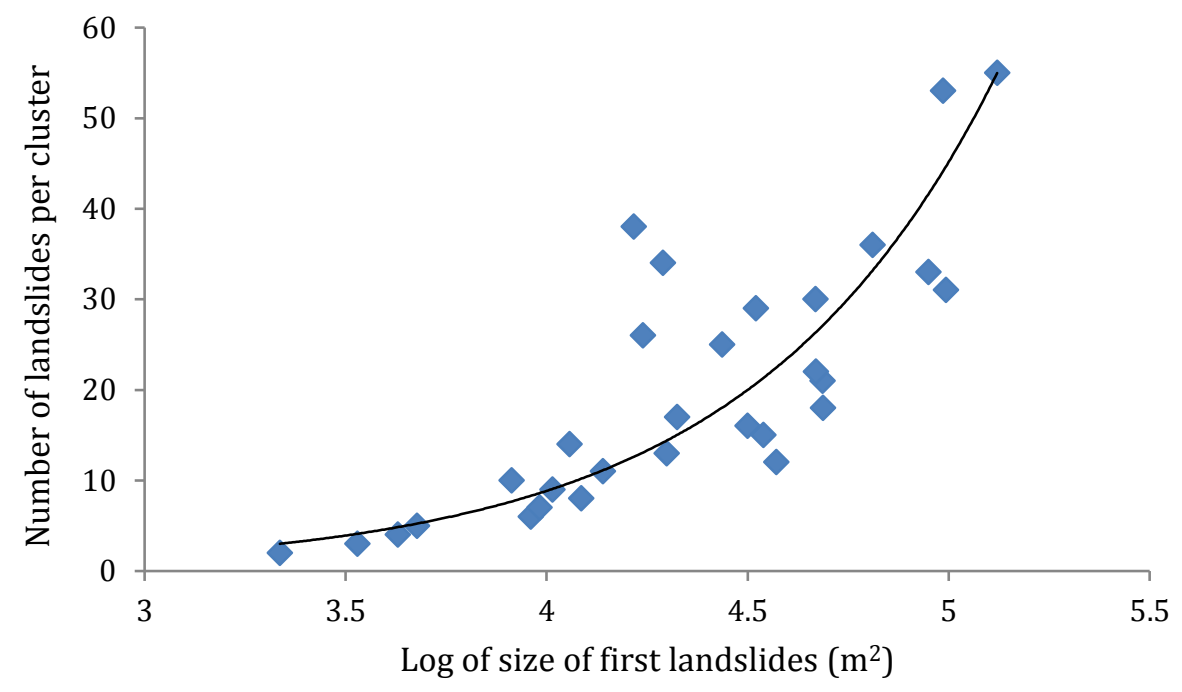

Figure 3.10. Relation between number of landslide versus size of earlier landslide per cluster.

\subsubsection{Implications for susceptibility to landslides}

The present study was designed to quantify the effect of landslide path dependency. This was already named "effect of previous landslides" and discussed to be a component of a time-variant landslide susceptibility (Samia et al. 2017) as:

Susceptibility $_{s, t}=f\left(\right.$ conditioning attributes $_{s}$, previous landslides $\left._{s, t}\right)$

We now quantified the time-variant component of susceptibility using the temporal dynamics of follow-up landslide fraction ( $\rho$, Eq. 1). These temporal dynamics were shown to follow an exponential decay. The most important characteristic numbers for our study area, that should be sufficiently generic to allow comparison with other study areas, are $b\left(\mathrm{y}^{-1}\right.$, the decay coefficient $)$ and $\rho_{a} / \rho_{0}(-$, the relative increase in landslide susceptibility immediately after an earlier landslide). In our case, $\rho_{a} / \rho_{0}$ is larger than 1 , indicating a positive effect of landslides on future landslides that decays over time. As suggested before (Samia et al. 2017), the opposite case, with $\rho_{a} / \rho_{0}<0$, would be expected in situations where landslides reduce the risk of follow-up landslides (such as where soils are thin and renewed weathering is needed before a new landslide is possible (Singh et al. 2008). 
A path dependent dynamic time-variant landslide susceptibility should be considered in addition to traditional susceptibility mapping where the influence of conditioning attributes in space is explored in a susceptibility mapping method (i.e., logistic regression). Then, an 'additional' time-dependent susceptibility term modifying the traditional susceptibility to landslides should be introduced to account for certain time changes after a landslide occurred. Using such a modelling approach based on coefficients of our exponential decay function $\left(\rho_{a}, b\right.$, and $\rho_{0}$, Eq. 2), we could determine which area (e.g. a slope unit (Carrara et al. 1991; Alvioli et al. 2016)) has recently experienced a landslide that may cause a follow-up landslide, quantify the increased probability to experience follow-up landslides and determine for how long this effect will persist.

\subsubsection{Implications for inventory requirements for landslides}

We found that geometric attributes (size and shape) of initial landslides have a strong effect on the likelihood of occurrence of follow-up landslides (Table 1) and the formation of landslide clusters (Figure 3.10). This had not been documented before, probably because in nearly all landslide inventories, the locations of landslides are mapped as points. We, therefore, recommend mapping of landslides with polygons either in field surveys or in remote sensing approaches (aerial photographs and satellite images) to allow determination of size and shape of landslides, and use this information to assess susceptibility to landslides.

For existing point-based multi-temporal inventories, quantification of landslide path dependency can be attempted if information about landslide area (in classes or in real numbers) is available. Under an assumption for landslide shape, this would allow the creation of an approximate landslide polygon, centred on the reported point-based location. From there, it can be determined whether landslides from subsequent time slices possibly overlap, follow-up landslide fraction ( $\rho$, Eq. 1) can be calculated and its temporal dynamics can be estimated. This procedure can be easily performed stochastically if estimates of the uncertainty in position, area and shape are available, yielding uncertainty bounds for $\rho$ and its temporal dynamics.

\subsubsection{Implications for regional planning}

Planning regulation in mountainous landslide prone areas depends on proper landslide susceptibility zonation of that territory. Given that the existing distribution of landslides in inventory maps of landslides is used to produce maps of landslide susceptibility, regular monitoring of the location and distribution of landslides is already required to keep maps of landslide susceptibility up to date for sustainable land use planning. Such regularly updated landslide inventory maps will also allow us to consider the short-term effect of existing landslides on followup landslide occurrence (Samia et al. 2017). Given that design timescales for buildings and infrastructure are usually longer than the $\sim 25$ years over which previous landslides meaningfully affect the susceptibility for future landslides, time-variant maps of susceptibility do not seem to be meaningful starting points for 
planning purposes. Maps of the time-variant portion of landslide susceptibility that are based on Eq. 3, however, are likely to show different patterns of susceptibility than traditional maps of susceptibility - and may, therefore, be valuable inputs to planning processes in regions that experience substantial follow-up landsliding.

\subsection{Conclusions}

We quantified the path dependent response of landslides. The path dependency between landslides exhibits an exponential response over time. In our study area, susceptibility increases by a factor of about 15 immediately after an earlier landslide and decreases to background levels of susceptibility over a period of 25 years. Larger and rounder landslides are more likely to be the cause of follow-up landsliding. The size of earlier landslides also plays an important role in forming landslide clusters. In addition, Topographic Wetness Index (TWI), absolute profile curvature, vertical distance to channel network, and relative slope position of a landslide co-determine the occurrence of follow-up landslides. A logistic regression model to predict follow-up landslide occurrence based on a combination of geometric and topographic attributes predicts follow-up landslide occurrence with $60 \%$ confidence. These findings can be used in a dynamic time-variant mapping of landslide susceptibility where changes in susceptibility levels occur over time based on exponential response of landslide path dependency. We stress the importance of multi-temporal polygon mapping and documenting of landslides to perform a time-variant assessment of susceptibility to landslides.

Acknowledgements. This work is part of $\mathrm{J}$ Samia $\mathrm{PhD}$ project at Wageningen university and Research, financed by the Ministry of Sciences, Research and Technology of Iran. We would like to thank two anonymous reviewers and the editor of Journal of Geomorphology Professor Markus Stoffel for their valuable and constructive comments in the preparation of final manuscripts. 



\section{Chapter 4}

\section{Implementing landslide path dependency in landslide susceptibility modelling}

This chapter is based on:

Samia, J., Temme, A., Bregt, A., Wallinga, J., Stuiver, J., Guzzetti, F., Ardizonne, F., Rossi, M. (2018). Implementing landslide path dependency in landslide susceptibility modelling. Landslides, 1-16, DOI: 10.1007/s10346-018-1024-y 


\begin{abstract}
Landslide susceptibility modelling - a crucial step towards the assessment of landslide hazard and risk - has hitherto not included the local, transient effects of previous landslides on susceptibility. In this contribution, we implement such transient effects, which we term 'landslide path dependency', for the first time. Two landslide path dependency variables are used to characterise transient effects: a variable reflecting how likely it is that an earlier landslide will have a follow-up landslide, and a variable reflecting the decay of transient effects over time. These two landslide path dependency variables are considered in addition to a large set of conditioning attributes conventionally used in landslide susceptibility. Three logistic regression models were trained and tested fitted to landslide occurrence data from a multi-temporal landslide inventory: 1) a model with only conventional variables, 2) a model with conventional plus landslide path dependency variables, and 3) a model with only landslide path dependency variables. We compare the model performances, differences in the number, coefficient and significance of the selected variables, and the differences in the resulting susceptibility maps. Although the landslide path dependency variables are highly significant and have impacts on the importance of other variables, the performance of the models and the susceptibility maps do not substantially differ between conventional and conventional plus path dependent models. The path dependent landslide susceptibility model, with only two explanatory variables, has lower model performance, and differently patterned susceptibility map than the two other models. A simple landslide susceptibility model using only DEM-derived variables and landslide path dependency variables performs better than the path dependent landslide susceptibility model, and almost as well as the model with conventional plus landslide path dependency variables - while avoiding the need for hard-tomeasure variables such as land use or lithology. Although the predictive power of landslide path dependency variables is lower than those of the most important conventional variables, our findings provide a clear incentive to further explore landslide path dependency effects and their potential role in landslide susceptibility modelling.
\end{abstract}




\subsection{Introduction}

Landslide susceptibility modelling is a key component in landslide hazard and risk assessment, crisis management and land use planning. Landslide susceptibility is the probability of landslide occurrence in an area based on a set of conditioning attributes (e.g., morphology, geology, soil) (Brabb 1984). In this context, landslide susceptibility is a time-invariant concept that purely provides an assessment of where a landslide is likely to occur (Guzzetti et al. 1999; Guzzetti et al. 2005). Hence, landslide susceptibility differs from landslide hazard which does consider the temporal probability of landslide occurrence (Varnes 1984; Guzzetti et al. 2005; Guzzetti et al. 2006a) and its magnitude (Guzzetti et al. 2005).

The availability of commercial and open source GIS, and of statistical software (Rossi et al. 2010; Rossi and Reichenbach 2016) has allowed many researchers to construct different empirical models for landslide susceptibility modelling. Direct geomorphological mapping, heuristic approaches and quantitative statistical models have all been used to model susceptibility to landslides. Within the category of quantitative statistical models, the last two decades landslide susceptibility modelling has been the playground for new data integration techniques including fuzzy logic (Saboya et al. 2006), artificial neural networks (Kawabata and Bandibas 2009), support vector machines (Kavzoglu et al. 2014), and random forests (Trigila et al. 2015). The various approaches applied in these models always involve estimating the relation between the presence or absence of landslides on the one hand, and a generally large set of conditioning attributes on the other hand. Performance of such models is usually assessed with a strong emphasis on Receiver Operating Characteristic (ROC) curves, and Area Under Curve (AUC) values (Mason and Graham 2002).

The temporal validity of predicted susceptibility levels in landslide susceptibility models have been considered indefinitely in all those approaches. However, there are indications from empirical studies that susceptibility levels are instead dynamic, such as the existence of a "relaxation time" of the landscape, following a major event triggering landslides. In the "relaxation time", the effects of external triggers (e.g., earthquake, rainfall) and also the strengths of ground change over time. These changes were demonstrated with the impacts of four earthquakes (MW 6.6-7.6) on the rate of landsliding. Marc et al. (2015) showed that the regional susceptibility of landsliding increases immediately after an earthquake, remains high for several months to years, and then returns to the background susceptibility level. This shows that landslide susceptibility levels are dynamic, and suggests that these changes need to be reflected in landslide susceptibility modelling.

We recently quantified the duration and strength of path dependency among landslides for the Collazzone study area in central Italy (Samia et al. 2017b; Samia et al. 2017a). Path dependency is a concept from complex system theory stating that the history of a system partly determines the future state of the system (Phillips 2006). In landsliding, path dependency means that the history of landslides at a certain location affects the susceptibility of future landslides at or near that location (Samia et al. 2017b). We found that in our study area earlier landslides locally, 
temporarily and positively affect the susceptibility to future landslides. Susceptibility rises immediately after a first landslide, and then decays to the original susceptibility values over a period of about two decades in the vicinity of existing landslides (Samia et al. 2017b; Samia et al. 2017a). These results led us to propose the concept of time-variant landslide susceptibility modelling in which a space-time dynamic component reflecting landslide history is added to the purely spatial conditioning attributes that have conventionally been used in landslide susceptibility modelling (Samia et al. 2017b; Samia et al. 2017a).

Our current work presented in this paper includes - for the first time - landslide path dependency in landslide susceptibility modelling, and compares results with conventional landslide susceptibility modelling. To do this, we use a detailed multitemporal landslide inventory containing 16 time slices of mapped landslides from the Collazzone study area in central Umbria, Italy (Guzzetti et al. 2006a; Ardizzone et al. 2013).

\subsection{Study area and data}

\subsubsection{Study area}

The hilly Collazzone study area, in central Umbria, Italy, covers an area of $78.9 \mathrm{~km}^{2}$ (Figure 4.1). The elevation in the area ranges from 145 to $634 \mathrm{~m}$ above sea level, and slope varies between 0 and $64^{\circ}$ derived from a Digital Terrain Model. Climate is Mediterranean with annual average precipitation of $885 \mathrm{~mm}$, and snow falls every 2 to 3 years (Rossi et al. 2010). Both forms of precipitation trigger landslides in the area (Guzzetti et al. 2006a). The majority (57\%) of the area is used as arable land. Forests, urban areas, pastures and vineyards are other substantial land uses. Soils have fine to medium textures and their thicknesses vary from a few centimetres to more than 1 meter (Rossi et al, 2010). A full description of study area can be found in (Guzzetti et al. 2006b; Galli et al. 2008; Samia et al. 2017b). 


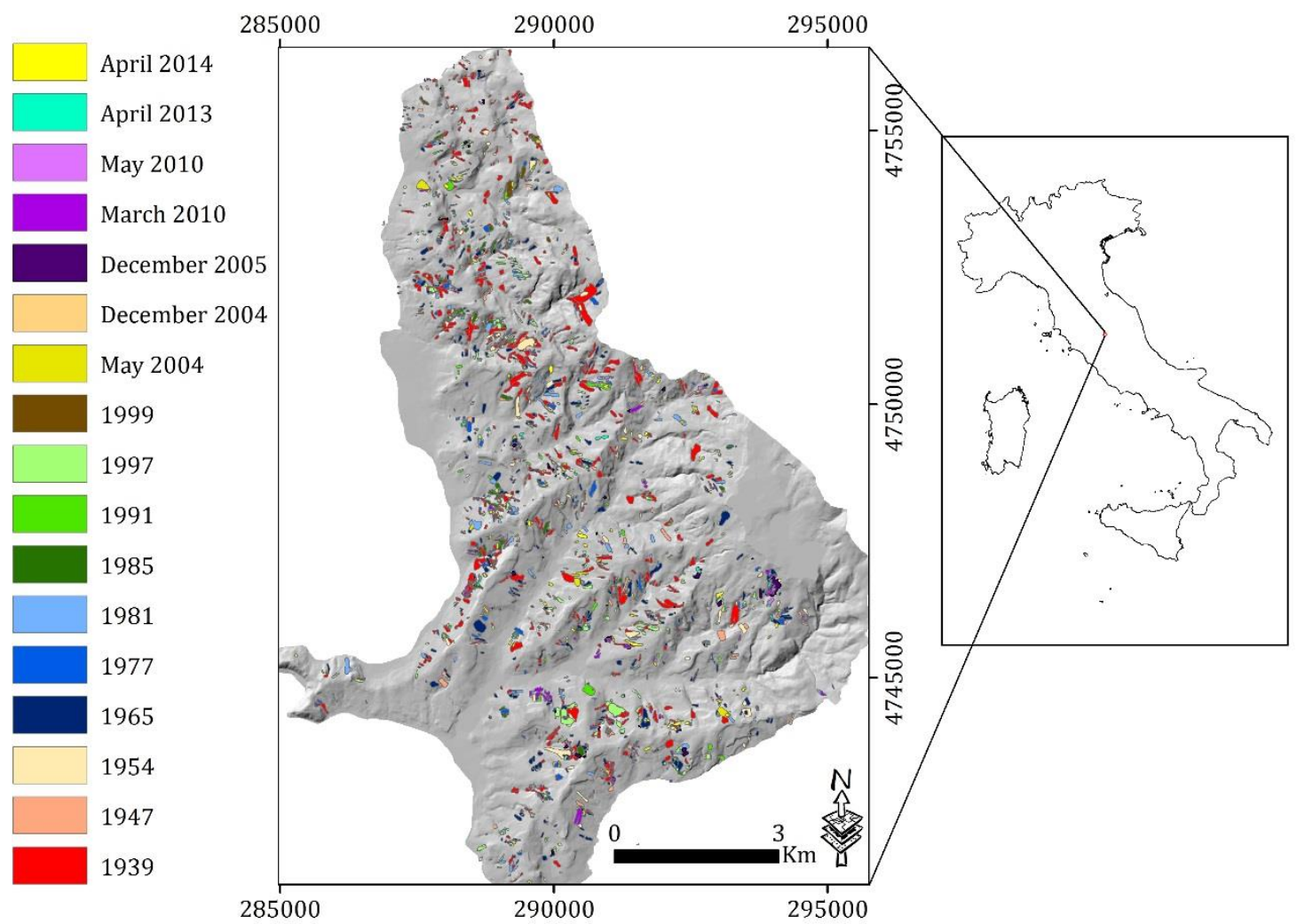

Figure 4.1. Multi-temporal landslide inventory including 16 time slices of landslide distribution overlaying a shaded relief image (left map) (adapted from (Samia et al. 2017b)). Location of Umbria region and of the Collazzone study area (right map). The coordinate system of both maps is EPSG:32633 (www.spatialreference.org). Note that the time slice from 1939 was only used to compute landslide path dependency variables, and not in landslide susceptibility modelling.

\subsubsection{Multi-temporal landslide inventory}

The Collazzone study area is active in terms of landslide occurrence. Landslides are regularly mapped and monitored using interpretation of aerial photographs, direct field mapping after major external triggers (e.g., intense rainfall and snowmelt), and also remote sensing with stereo couples of GEOEYE and Worldview images (Ardizzone et al. 2013). A multi-temporal landslide inventory based on these sources is available for the study area, containing 3391 landslides mapped in 19 different time slices. All landslides in the multi-temporal landslide inventory are shallow and deep-seated landslides (Guzzetti et al. 2006b). The first three time slices where the dates of previous landslides are not well-constrained, were not used in this study. Therefore, the multi-temporal landslide inventory that is used in this work contains 16 time slices with a total of 2383 landslides (Figure 4.1). The time slices range from landslides in 1947 to landslides in April 2014. A detailed description of the multi-temporal landslide inventory, with information about the 
preparation and mapping of time slices of landslides inventories, age of landslides, spatial and temporal uncertainty of mapped landslides and frequency area relation of the multi-temporal landslide inventory is given in (Guzzetti et al. 2006b; Samia et al. 2017b).

\subsubsection{Mapping unit for landslide susceptibility modelling}

A subdivision of the study area into mapping units (Figure 4.2) i.e., morphohydrological subdivisions of terrain containing a set of conditions different from neighbouring units, is common for the preparation of landslide susceptibility maps (Carrara et al. 1995; Guzzetti et al. 1999; Guzzetti et al. 2006b; Alvioli et al. 2016). We used a set of previously defined "slope units" that divide the study area into hydrological regions bounded by drainage and divide lines (Carrara et al. 1991; Alvioli et al. 2016). These slope units have proven to be a reliable unit to map susceptibility to landslides in our study area (Guzzetti et al. 2006a; Guzzetti et al. 2006b) and more generally in the Umbria region in Italy (Carrara et al. 1991; Guzzetti et al. 1999; Cardinali et al. 2002). In total, 894 slope units were identified for our study area. Along with the preparation of slope units, 30 morphological and hydrological parameters (Table 4.1) were created that are part of the set of conditioning attributes used in this work. A detailed description regarding the preparation of slope units and their use for susceptibility modelling can be found in (Carrara et al. 1991; Rossi et al. 2010).

\subsubsection{Conditioning attributes}

To classify the slope units according to their susceptibility to landslide, we used the same set of 51 conditioning attributes as previous work (Rossi et al. 2010). This set (Table 1) includes 24 morphological, six hydrological, nine lithological (Figure 4.2), three structural, and eight land use classes (Figure 4.2), and one attribute showing the presence of ancient deep-seated landslides. A detailed description of the preparation of these attributes and their importance in landslide susceptibility mapping can be found in (Guzzetti et al. 2006a; Guzzetti et al. 2006b; Rossi et al. 2010). For this study, additional variables reflecting landslide path dependency were calculated. These variables describe the spatial probability of earlier landslide causing follow-up landslides and landslide susceptibility temporal decay (Table 4.1 and see section 4.3.1). 

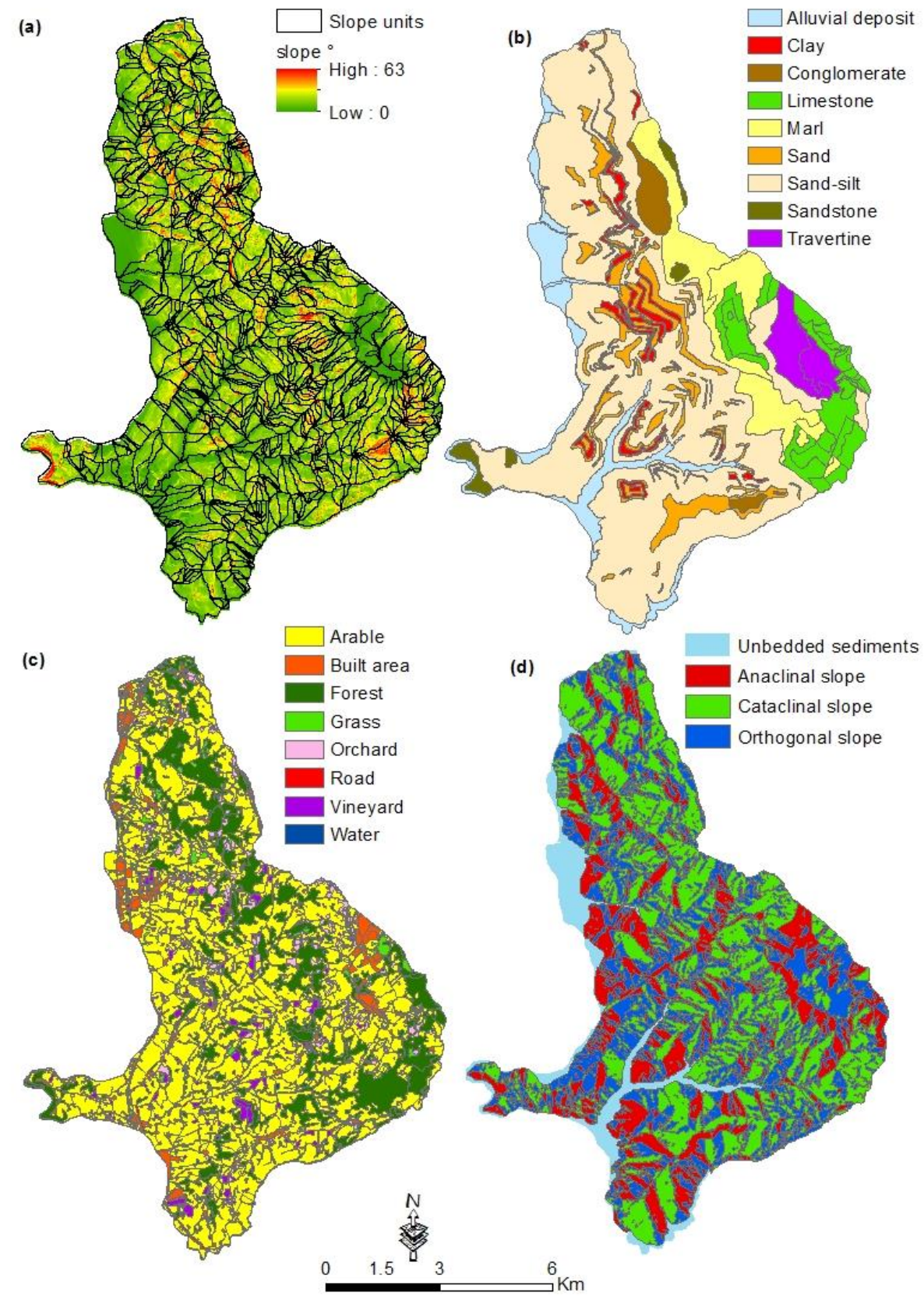

Figure 4.2. Slope units and slope (a), geology (b), land use (c), and bedding attitude with respect to slope (d) in the Collazzone study area. 
Table 4.1. Conditioning attributes used in conventional landslide susceptibility modelling (Rossi et al. 2010) and landslide path dependency variables.

\begin{tabular}{|c|c|}
\hline Category & Conditioning attributes (independent variables or classes) \\
\hline Land use & $\begin{array}{l}\text { Arable, built area, forest, grass, orchard, road, vineyard, } \\
\text { water }\end{array}$ \\
\hline Old landslides & Deposit of ancient deep-seated landslide \\
\hline Lithology & $\begin{array}{l}\text { Recent alluvial deposit, clay, conglomerate, limestone, marl, } \\
\text { sand, sand-silt, sandstone, travertine }\end{array}$ \\
\hline Slope direction & $\begin{array}{l}\text { Slope unit facing N-NE, slope unit facing S-SE, slope unit } \\
\text { facing S-SW, concave profile downslope, concave-convex } \\
\text { profile downslope, downslope concave slope, slope unit } \\
\text { mean terrain gradient, slope unit rectilinear profile, slope } \\
\text { unit slope (lower portion), slope unit terrain gradient } \\
\text { (intermediate portion), slope unit terrain gradient (upper } \\
\text { portion), slope unit terrain gradient standard deviation, } \\
\text { slope unit with convex slope (downslope profile), slope unit } \\
\text { with convex-concave slope (downslope profile), slope unit } \\
\text { with irregular slope (downslope profile), aspect (D8 } \\
\text { notation), }\end{array}$ \\
\hline Hydrological & $\begin{array}{l}\text { Drainage basins total area upstream the slope unit, drainage } \\
\text { channel mean slope, slope unit drainage channel length, } \\
\text { slope unit drainage channel magnitude, slope unit drainage } \\
\text { channel order, slope unit area }\end{array}$ \\
\hline Geomorphological & $\begin{array}{l}\text { Standard deviation of terrain unit length, slope unit } \\
\text { elevation standard deviation, slope unit length, slope unit } \\
\text { mean elevation, slope unit surface roughness index, } \\
\text { maximum orientation of the slope unit, minimum } \\
\text { orientation of the slope unit, mean slope angle of the slope } \\
\text { unit squared }\end{array}$ \\
\hline Structural & Anaclinal slope, cataclinal slope, orthogonal slope \\
\hline Landslide path & Susceptibility temporal decay, spatial probability of earlier \\
\hline dependency & landslides causing follow-up landslides \\
\hline
\end{tabular}




\subsection{Methods}

We compared (i) conventional landslide susceptibility modelling, (ii) conventional plus path dependent landslide susceptibility modelling, and (iii) path dependent landslide susceptibility modelling using a forward conditional Multiple Logistic Regression (MLR) (Figure 4.3). We assessed the performance of all three models with Area Under Curve (AUC) values of the Receiver Operating Characteristics (ROC) curve. Then, we compared the coefficients estimated in landslide susceptibility models. Finally, we compared the predicted susceptibility maps.

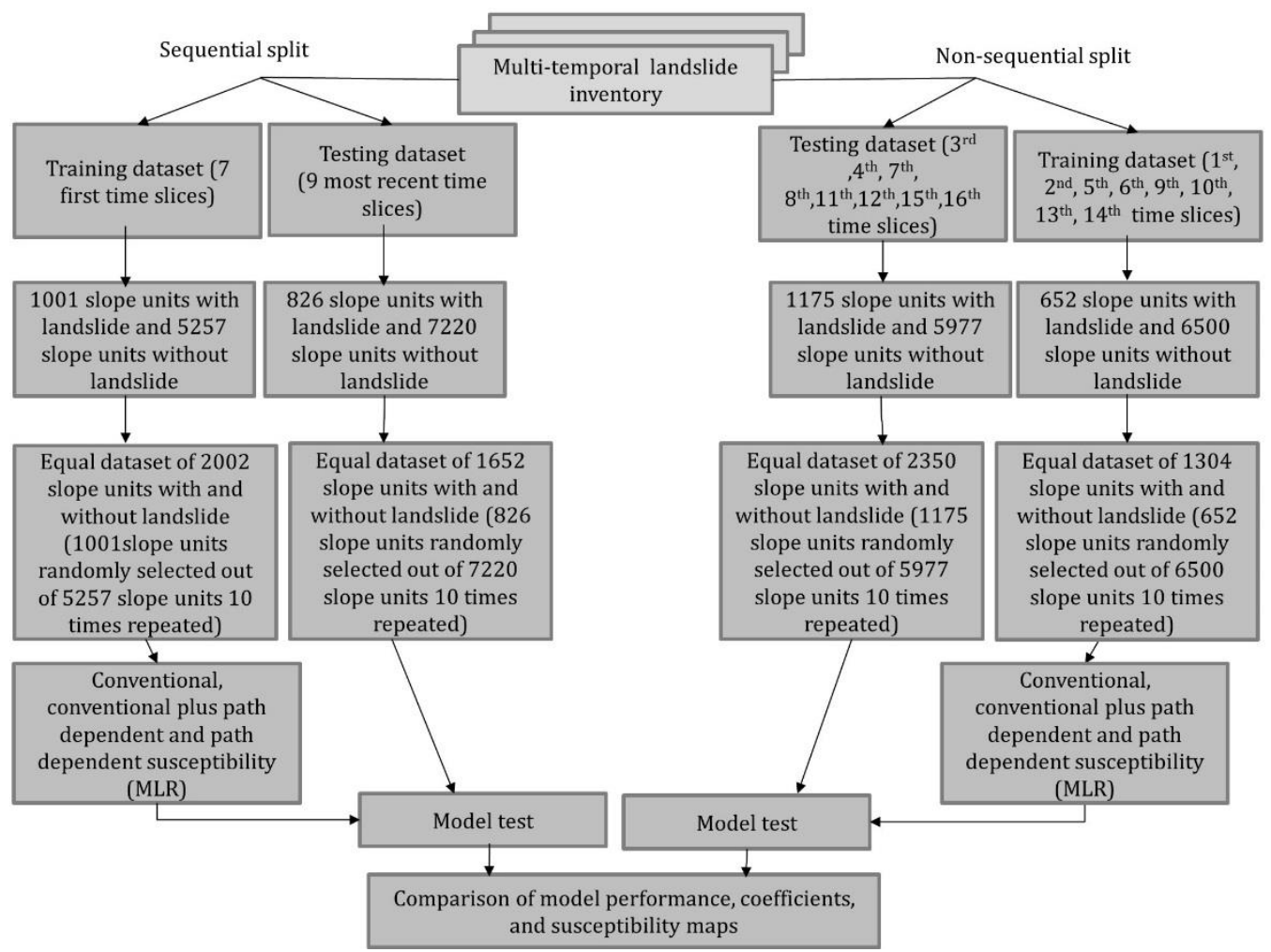

Figure 4.3. Flowchart of methods used. For difference between sequential split and nonsequential split see section 4.3.2.

\subsubsection{Landslide path dependency variables}

We computed four landslide path dependency variables in an attempt to reflect the history of landslides from the multi-temporal landslide inventory (Figure 4.1). The first path dependency variable, called susceptibility temporal decay (Table 1) reflects that the additional local susceptibility due to an earlier landslide decays exponentially, following:

Susceptibility temporal decay $=e^{(b * t)}$ 
For every slope unit in each time slice, we calculated the susceptibility temporal decay depending on when a landslide last time happened in the slope unit, regardless of where in the slope unit the landslide happened. The susceptibility temporal decay values range from 0 to 1 . Values close to 1 indicate that landslides happened recently in the slope unit and values close to 0 indicate that the most recent landslide happened a long time ago. This was based on our earlier finding of exponential decay in the number of landslides geographically overlapping with earlier landslides (Samia et al. 2017a). We found that for the Collazzone study area the susceptibility is raised immediately after an earlier landslide by a factor of 15 , and then it decreases over time with an exponential coefficient value of $b=-0.12 \pm$ $0.01 \mathrm{y}^{-1}$ (Figure 4.). The second variable (Table 4.1) is the spatial probability of earlier landslides causing follow-up landslides, which was quantified according to geometric and topographic attributes of earlier landslides (Samia et al. 2017a). The third variable is the sum of spatial probability of all landslides that may have happened in the most recent time slice experiencing follow-up landslides. The fourth variable is an aggregated number combining the probability of follow-up landslides of all known earlier landslides under the assumption that susceptibility decays exponentially $(b=-0.12)$ as following:

$$
\text { Aggregated probability }=
$$

$\sum_{\text {Earlier slide } i}^{\text {Earle }}$ probability of earlier landslide ${ }_{i}$ causing follow - up landslide $* e^{(b * t)}$

Time differences, spatial association among landslides, geometric and topographic attributes of landslides were the key elements to calculate all these four variables.

Only the first two of these landslide path dependency variables were used in landslide susceptibility modelling because the third and fourth variables were very strongly correlated with the second variable $(r>0.6)$ for our dataset. However, they may be less correlated and hence useful in other settings with multi-temporal landslide inventory. 


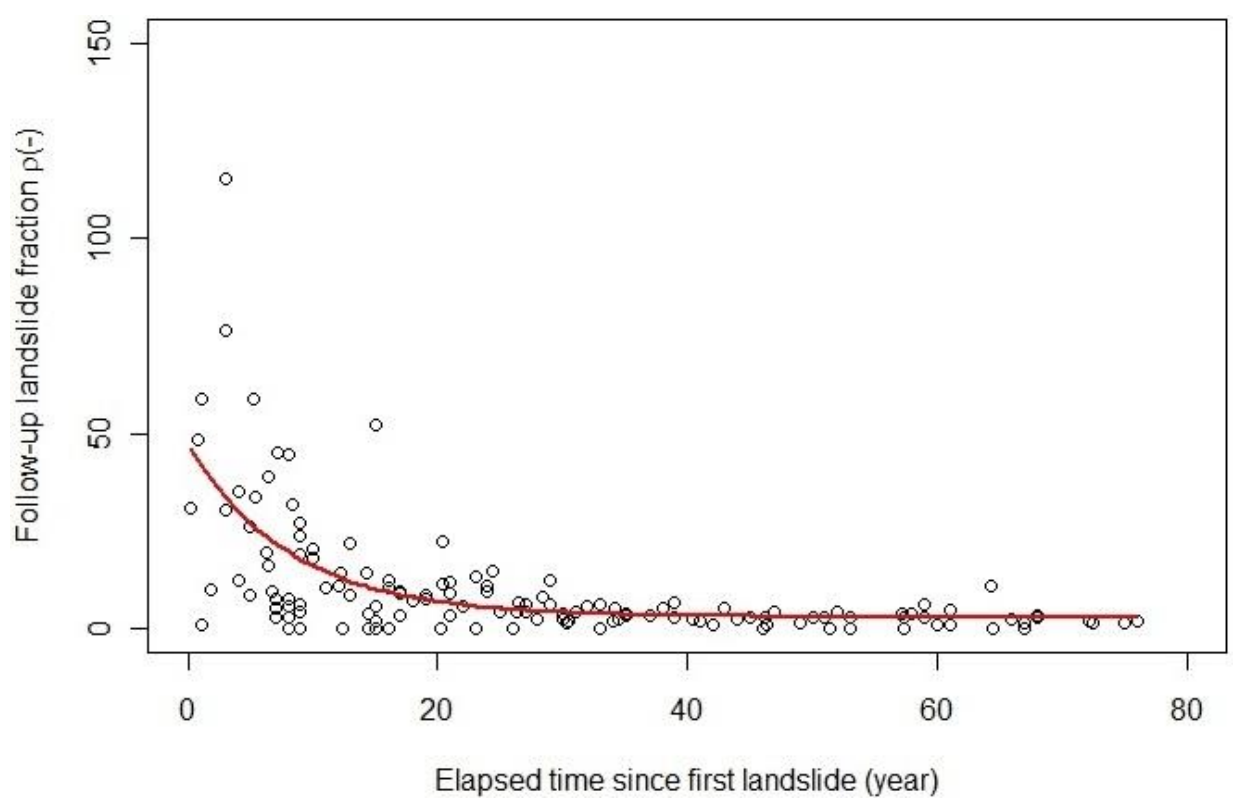

Figure 4.4. Temporal response of landslide path dependency with an exponential decay (Samia et al. 2017a).

\subsubsection{Sequential and non-sequential splitting of multi-temporal landslide inventory for training and testing}

Usually, in landslide susceptibility modelling, a mono-temporal landslide inventory (i.e. a geomorphological) (Guzzetti et al. 2012) is divided randomly into a training and a testing dataset, with a ratio of $70 \%$ of the data for training and $30 \%$ of the data for validation (Tien Bui et al. 2016). In previous studies that used almost the same multi-temporal landslide inventory as we do in this work, older time slices have been used for training, and younger time slices for testing (Rossi et al. 2010). We named this approach 'sequential splitting' (Figure 4.3, Table 4.2) and we used it as a first splitting approach in this work, with the 7 oldest time slices from 1947 to 1991 (covering a period of 44 years) used for training , and the 9 most recent time slices from 1997 to 2014 (covering a period of 17 years) used for testing.

However, this method of splitting is not well suited where an estimation of the effects of landslide path dependency is required. Note that the landsliding history of the area is not known before the earliest time slice in 1939. That means that for this time slice, the value of the temporal decay susceptibility (Table 4.1) is unknown, and that also subsequent old time slices may miss an important part of the landslide history of the area. Thus, sequential splitting leads to a narrower distribution of times since previous landslides in the training dataset than in the testing dataset. A model based on the training dataset may hence be uncertain about the role of the time passed since a previous landslide, especially for longer 
times passed. Additionally, in recent times, time slices are closer together in time due to more frequent availability of high quality remote sensing imagery. The corresponding lack of very short temporal separations in the training dataset will hamper accurate estimation of the role of time as well. More generally, the path dependency paradigm creates room for thought that the conventional spacefocussed susceptibility rules change over time. If so, a model trained on an old dataset may not be accurate for a recent dataset.

For these reasons, we also employed a 'non-sequential splitting' approach, which to our knowledge is novel in the realm of landslide susceptibility modelling. The non-sequential selection of time slices from the multi-temporal landslide inventory (Figure 4.1, Table 4.2) prioritizes an equal range of temporal separations between time slices in the training and the testing datasets, to avoid the disadvantages mentioned above. On the downside, this approach makes it more difficult to have approximately equal numbers of slope units with and without landslides in both datasets. For the training dataset, the $1^{\text {st }}, 2^{\text {nd }}, 5^{\text {th }}, 6^{\text {th }}$, $9^{\text {th }}, 10^{\text {th }}, 13^{\text {th }}$ and $14^{\text {th }}$ time slices were used (covering a period of 63 years). Note that the first time slice here refers to 1947 - the first time slice for which a partial history of landsliding is known. For the testing dataset, the $3^{\text {rd }}, 4^{\text {th }}, 7^{\text {th }}, 8^{\text {th }}, 11^{\text {th }}$, $12^{\text {th }}, 15^{\text {th }}$ and $16^{\text {th }}$ time slices were used (covering a period of 49 years) (Table $4.2)$.

Table 4.2. Sequential and non-sequential splitting of time slices of the multi-temporal landslide inventory to be used in conventional, conventional plus path dependent and purely path dependent landslide susceptibility modelling

\begin{tabular}{|c|c|c|}
\hline $\begin{array}{l}\text { Selection of } \\
\text { training and testing } \\
\text { datasets }\end{array}$ & $\begin{array}{l}\text { Time slices in the } \\
\text { training dataset }\end{array}$ & $\begin{array}{l}\text { Time slices in the } \\
\text { testing dataset }\end{array}$ \\
\hline Sequential splitting & $\begin{array}{l}\text { 1947, 1954, 1965, 1977, } \\
1981,1985,1991\end{array}$ & $\begin{array}{l}\text { 1997, 1999, May 2004, Dec 2004, } \\
\text { Dec 2005, March 2010, May 2010, } \\
\text { April 2013, April } 2014\end{array}$ \\
\hline $\begin{array}{l}\text { Non-sequential } \\
\text { splitting }\end{array}$ & $\begin{array}{l}\text { 1947, 1954, 1981, 1985, } \\
\text { 1999, May 2004, March } \\
\text { 2010, May } 2010\end{array}$ & $\begin{array}{l}\text { 1965, 1977, 1991, 1997, Dec 2004, } \\
\text { Dec 2005, April 2013, April } 2014\end{array}$ \\
\hline
\end{tabular}

\subsubsection{Multiple logistic regression model}

Logistic regression is the most widely used statistical model in landslide susceptibility modelling (Mancini et al. 2010; Martinović et al. 2016). In logistic regression, a set of explanatory (independent) variables explains variation in the 
binary dependent variable (Menard 2000). In landslide susceptibility modelling, explanatory variables are e.g., slope and geology, and the dependent variable is the presence or absence of landslides in the mapping unit of choice. The relation between independent variables and dependent variable is used to classify the mapping units (slope unit or pixel) of an area to different levels of susceptibility to landslides. In this context, each mapping unit has a probability of landslide occurrence $(\rho)$ in the range from 0 to 1 (Martinović et al. 2016):

$\rho=\ln \left(\frac{1}{1+e^{-z}}\right)$

where $\mathrm{z}$ is a linear combination of coefficients related to independent variables selected by logistic regression according to their importance and significance reflected as:

$z=\beta_{0}+\beta_{1} X_{1}+\beta_{2} X_{2}+\cdots+\beta_{n} X_{n}$

where $\beta_{0}$ is the intercept of the model, $\beta_{1}, \beta_{2}$ and $\beta_{\mathrm{n}}$ are the coefficients of independent variables, and $\mathrm{X}_{1}, \mathrm{X}_{2}, \mathrm{X}_{\mathrm{n}}$ are independent variables selected by the model.

\subsubsection{Landslide susceptibility modelling}

Conventional landslide susceptibility modelling was performed using a set of 51 previously used conditioning attributes (Rossi et al, 2010) (Table 4.1). The conventional plus path dependent landslide susceptibility modelling was performed using the same 51 conditioning attributes, plus spatial probability of earlier landslides causing follow-up landslides and susceptibility temporal decay variables describing landslide path dependency (Table 4.1). Path dependent landslide susceptibility modelling was performed using only the two new landslide path dependency variables (Table 4.1). The number of slope units without landslides is more than the number of slope units with landslides (Figure 4.3). To make a dataset with equal numbers of slope units with and without landslides, all slope units with landslides and a random but equal number of slope units without landslides were selected. To explore the effect of this random selection, it was repeated 10 times. After preparation of the 10 training datasets with sequential splitting, and 10 training datasets with non-sequential splitting, we applied logistic regression to all twenty. We imposed an entry probability of 0.05 and a removal probability of 0.06 to reduce the risk of overfitting in the model. To avoid multicollinearity, we allowed only inter-variable correlations less than 0.6. Then, the contingency tables were computed to show the True Positive (slope units with landslide and predicted with landslide), True Negative (slope units without landslide and predicted without landslide), False Positive (slope units without landslide but predicted with landslide) and False Negative (slope unites with landslide and predicted without landslide) (Jolliffe and Stephenson 2003). Finally, the Area Under Curve values (AUC), quantifying the accuracy of performance of predicted models (Mason and Graham 2002; Fawcett 2006), and the Akaike Information Criterion (AIC) which quantifies the goodness of fit while penalising for the complexity of the model (Akaike 1974; Petschko et 
al. 2012) were computed for the training datasets. To test the models, the models were applied to the 20 testing datasets, and again contingency tables and the AUC values for these testing datasets were calculated. The coefficients of variables selected by logistic regression in conventional landslide susceptibility were compared with the coefficients of variables selected by logistic regression in conventional plus path dependent landslide susceptibility. Finally, we averaged the probability of landslide occurrence in the 10 training and 10 testing datasets, and used this to map susceptibility to landslides.

\section{$4.4 \quad$ Results}

\subsubsection{Model performance}

In our test case, conventional plus path dependent landslide susceptibility modelling resulted in similar model performance to conventional landslide susceptibility modelling. This was true for both sequential and non-sequential splitting of the multi-temporal landslide inventory (Table 4.3). In sequential splitting, the best training result was obtained with conventional plus path dependent landslide susceptibility modelling with highest AUC $=0.775 \pm 0.006$ (Table 4.3, Figure 4.5) and lowest Akaike Information Criterion (AIC) $=2281 \pm 23$. In non-sequential splitting, the best training result was obtained again with conventional plus path dependent landslide susceptibility modelling with highest AUC $=0.767 \pm 0.007$ and lowest $\mathrm{AIC}=1515 \pm 15$.

The best testing result was obtained with conventional plus path dependent landslide susceptibility for the non-sequential splitting, with AUC $=0.754 \pm 0.012$ (Table 4.3 and Figure 4.5). The landslide susceptibility model using only landslide path dependency variables performed acceptably as well, with best AUC $=0.688 \pm$ 0.009 for training in non-sequential splitting, and AUC $=0.682 \pm 0.022$ for testing in the sequential splitting (Table 4.3).

The clearest difference between these three landslide susceptibility models is that the testing results are higher or closer to the training results when only using landslide path dependency variables. In general, non-sequential splitting has larger differences between model performance and testing (Table 4.3). 
Table 4.3. Area Under Curve (AUC) and Akaike Information Criterion (AIC) values in conventional, conventional plus path dependent and path dependent landslide susceptibility modelling in sequential and non-sequential splitting.

\begin{tabular}{|c|c|c|c|c|c|c|}
\hline \multirow{2}{*}{$\begin{array}{l}\text { AUC and } \\
\text { AIC } \\
\text { values }\end{array}$} & \multicolumn{2}{|c|}{ Conventional susceptibility } & \multicolumn{2}{|c|}{$\begin{array}{l}\text { Conventional plus path } \\
\text { dependent susceptibility }\end{array}$} & \multicolumn{2}{|c|}{ Path dependent susceptibility } \\
\hline & Sequential & Non-sequential & Sequential & Non-sequential & Sequential & Non-sequential \\
\hline $\begin{array}{l}\text { AUC } \\
\text { training }\end{array}$ & $0.773 \pm 0.008$ & $0.763 \pm 0.008$ & $0.775 \pm 0.006$ & $0.767 \pm 0.007$ & $0.662 \pm 0.007$ & $0.688 \pm 0.009$ \\
\hline $\begin{array}{l}\text { AIC } \\
\text { training }\end{array}$ & $2287 \pm 26$ & $1523 \pm 23$ & $2281 \pm 23$ & $1515 \pm 15$ & $2586 \pm 16$ & $1671 \pm 13$ \\
\hline $\begin{array}{l}\text { AUC } \\
\text { testing }\end{array}$ & $0.730 \pm 0.007$ & $0.753 \pm 0.011$ & $0.733 \pm 0.012$ & $0.754 \pm 0.012$ & $0.682 \pm 0.022$ & $0.673 \pm 0.007$ \\
\hline
\end{tabular}
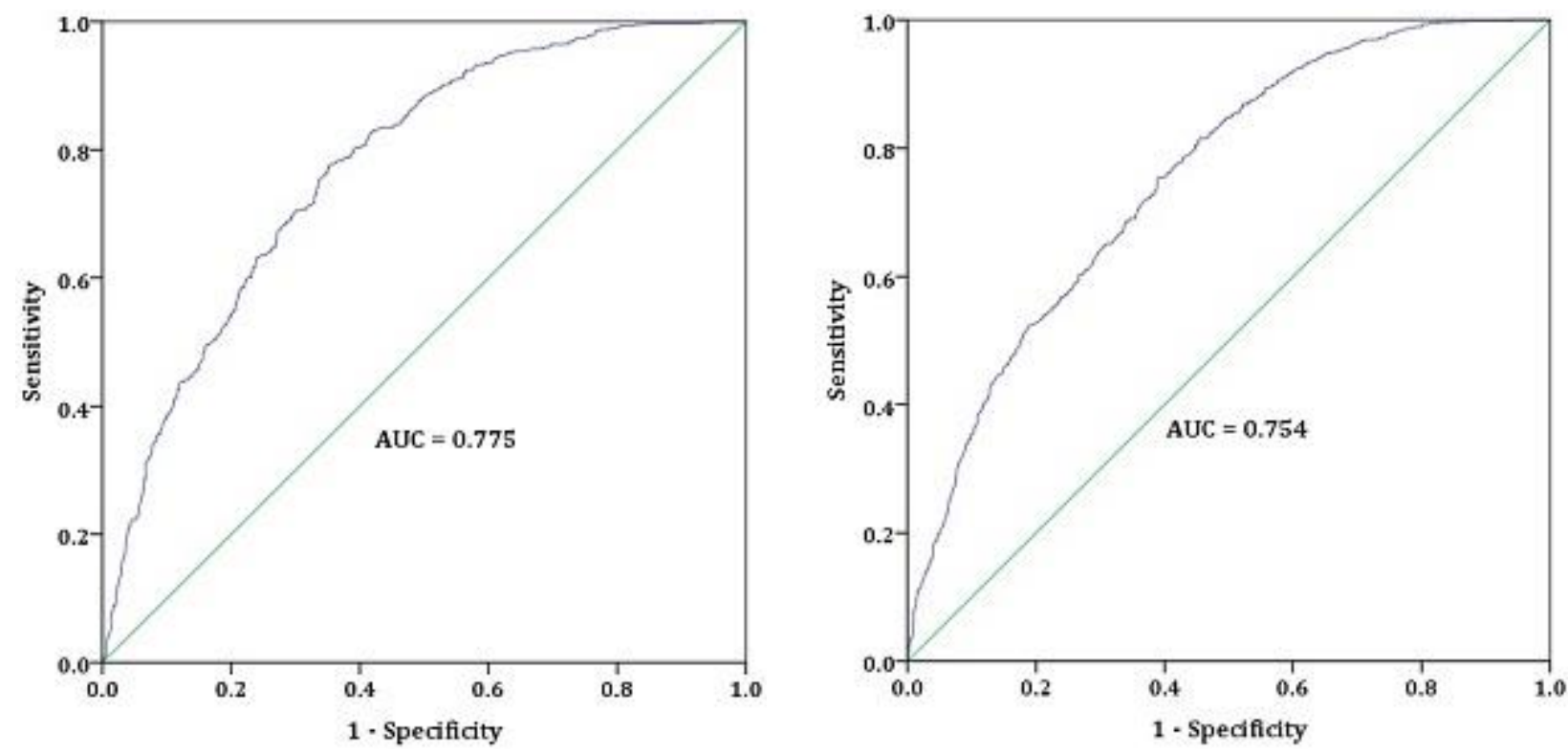

Figure 4.5. Examples of Receiver operating characteristic (ROC) curves with highest AUC values for training in conventional plus path dependent landslide susceptibility modelling for the sequential splitting (right) and for testing in conventional plus path dependent landslide susceptibility modelling for the non-sequential splitting (left).

The contingency tables values (Table 4.4) calculated with a probabilistic cut off value of 0.5 showed slight differences between conventional and conventional plus 
path dependent susceptibility, in sequential and non-sequential splitting. The path dependent susceptibility model differs substantially from these two models.

Table 4.4. Contingency table for conventional susceptibility, and conventional plus path dependent susceptibility and purely path dependent susceptibility in sequential and nonsequential splitting.

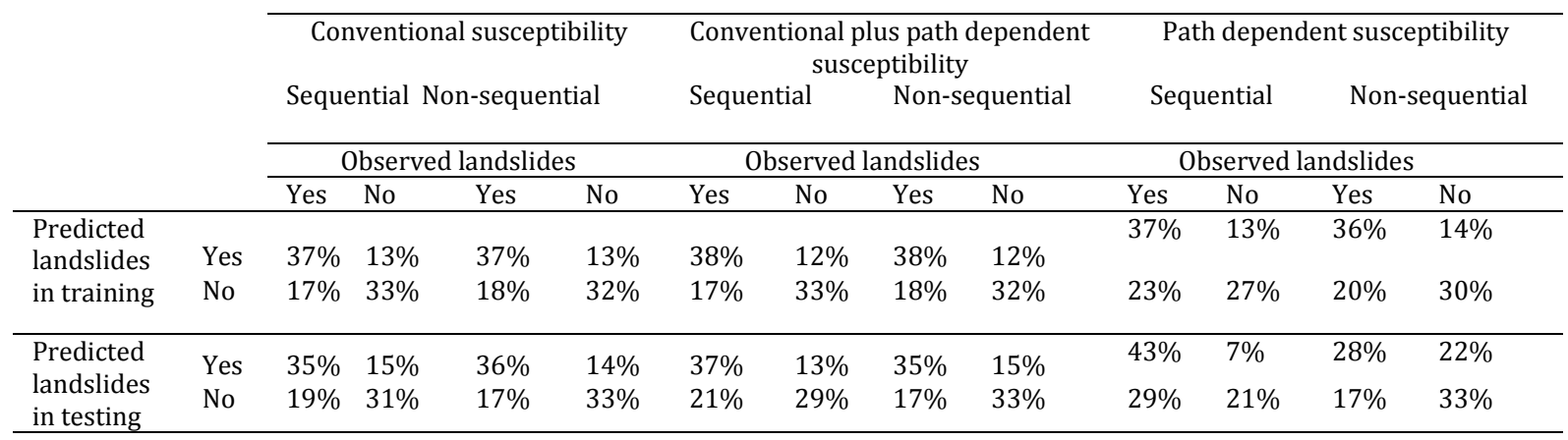

\subsubsection{Effect of landslide path dependency variables on variable selection}

The spatial probability that an earlier landslide will cause a follow-up landslide was selected as an explanatory variable (independent variable) in all 10 repetitions of conventional plus path dependent susceptibility modelling using sequential splitting, and in 8 out of 10 repetitions using non-sequential splitting (Table 4.5). In these 18 cases, the significance of this variable was always better than 0.0001 . The susceptibility temporal decay variable was selected 6 times, both in sequential and non-sequential splitting of conventional plus path dependent susceptibility (Table 4.5). In these 12 cases, susceptibility temporal decay variable was always significant as imposed during the training of the model $(\mathrm{p}<0.05)$. When using only the two path dependency variables, the spatial probability of earlier landslide causing follow-up landslide variable was always selected (i.e., 20 times), whereas susceptibility decay variable was selected 6 times in sequential and 10 times in nonsequential splitting. These variables were significant $(p<0.05)$ in all 36 cases as well. The importance of the landslide path dependency variables is also shown by their effects on the number and coefficients of variables selected by the model (Table 4.5, Figure 4.6 and Figure 4.7). 
Table 4.5. Variables selected by logistic regression in conventional susceptibility, conventional plus path dependent susceptibility and path dependent susceptibility. Only variables included 6 or more times out of 10 repetitions are reported. Numbers between parentheses indicate the number of times that variables were selected in the 10 repetitions.

\begin{tabular}{|c|c|c|c|}
\hline & $\begin{array}{l}\text { Landslide } \\
\text { susceptibility }\end{array}$ & $\begin{array}{l}\text { Variables selected by logistic regression in } 10 \text { times repetition } \\
\text { of logistic regression model }\end{array}$ & $\begin{array}{l}\text { Average } \\
\text { number } \\
\text { of } \\
\text { variables } \\
\text { in the } \\
\text { model }\end{array}$ \\
\hline \multirow{3}{*}{ 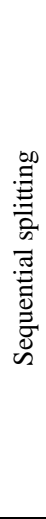 } & $\begin{array}{l}\text { Conventional (51 } \\
\text { variables } \\
\text { available) }\end{array}$ & $\begin{array}{l}\text { Limestone (10), forested area (10), slope unit area (10), } \\
\text { minimum orientation of the slope Unit (10), cultivated area } \\
\text { with trees (9), slope unit mean terrain gradient (8), slope unit } \\
\text { length (8), mean slope angle of the slope unit squared (8), } \\
\text { water bodies (7), recent alluvial deposit (6), marl (6), slope } \\
\text { unit mean elevation (6), slope unit mean terrain gradient (6) }\end{array}$ & 13.7 \\
\hline & $\begin{array}{l}\text { Conventional plus } \\
\text { path dependent ( } 53 \\
\text { variables } \\
\text { available) }\end{array}$ & $\begin{array}{l}\text { Spatial probability of earlier landslide causing follow-up } \\
\text { landslide (10), limestone (10), forested area (10), slope unit } \\
\text { area (10), minimum orientation of the slope unit (10), } \\
\text { cultivated area with trees (9), recent alluvial deposit (7), } \\
\text { susceptibility decay (6), water bodies (6), slope unit mean } \\
\text { elevation (6), slope unit mean terrain gradient (6), mean slope } \\
\text { angle of the slope unit squared (6) }\end{array}$ & 14 \\
\hline & $\begin{array}{l}\text { Path dependent } \\
\text { (two variables } \\
\text { available) }\end{array}$ & $\begin{array}{l}\text { Spatial probability of earlier landslide causing follow-up } \\
\text { landslide (10), susceptibility temporal decay (6) }\end{array}$ & 1.6 \\
\hline \multirow{3}{*}{ 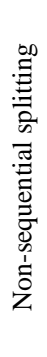 } & $\begin{array}{l}\text { Conventional (51 } \\
\text { variables } \\
\text { available) }\end{array}$ & $\begin{array}{l}\text { Limestone (10), recent alluvial deposit (9), cultivated area (8), } \\
\text { slope unit length (7), gravel and coarse continental sediments } \\
(6) \text {, pasture (6), slope unit elevation standard deviation (6), } \\
\text { slope unit length (6) }\end{array}$ & 10.7 \\
\hline & $\begin{array}{l}\text { Conventional plus } \\
\text { path dependent ( } 53 \\
\text { variables } \\
\text { available) }\end{array}$ & $\begin{array}{l}\text { Limestone (10), spatial probability of earlier landslide causing } \\
\text { follow-up landslide (8), slope unit elevation standard } \\
\text { deviation (8), recent alluvial deposit (8), slope unit length (7), } \\
\text { cultivated area (7), susceptibility temporal decay (6) }\end{array}$ & 11 \\
\hline & $\begin{array}{l}\text { Path dependent } \\
\text { (two variables } \\
\text { available) }\end{array}$ & $\begin{array}{l}\text { Spatial probability of earlier landslide causing follow-up } \\
\text { landslide (10), susceptibility temporal decay (10) }\end{array}$ & 2 \\
\hline
\end{tabular}

In sequential splitting, for conventional susceptibility, on average 13.7 variables and for conventional plus path dependent susceptibility 14 variables were selected (Table 4.5). Also, in non-sequential splitting the conventional landslide susceptibility selected on average 10.7 variables and the conventional plus path dependent susceptibility selected 11 variables on average. With adding the two landslide path dependency variables into the conventional landslide susceptibility, the inclusion and exclusion of other variables also changed. These were seen both in sequential and non-sequential splitting. In conventional plus path dependent susceptibility in sequential splitting, on average 3.25 variables were removed and 2.7 variables were added. In conventional plus path dependent susceptibility in non-sequential splitting, on average 3.5 variables were removed and 3.1 variables were added. In path dependent susceptibility, for the sequential splitting on average 1.6 variables (out of two landslide path dependency in 10 times repetition) 
were selected, and for non-sequential splitting both landslide path dependency variables were selected in all of the10 times repetition (Table 4.5).

The regression coefficients of variables that were present in both the conventional and the conventional plus path dependent models changed slightly (less than $10 \%$ ) with sequential splitting (Figure 4.6). The largest change is for recent alluvial deposit; around 11\%. With non-sequential splitting, limestone and cultivated area changed, about $12 \%$ and $14 \%$ respectively (Figure 4.7 ).

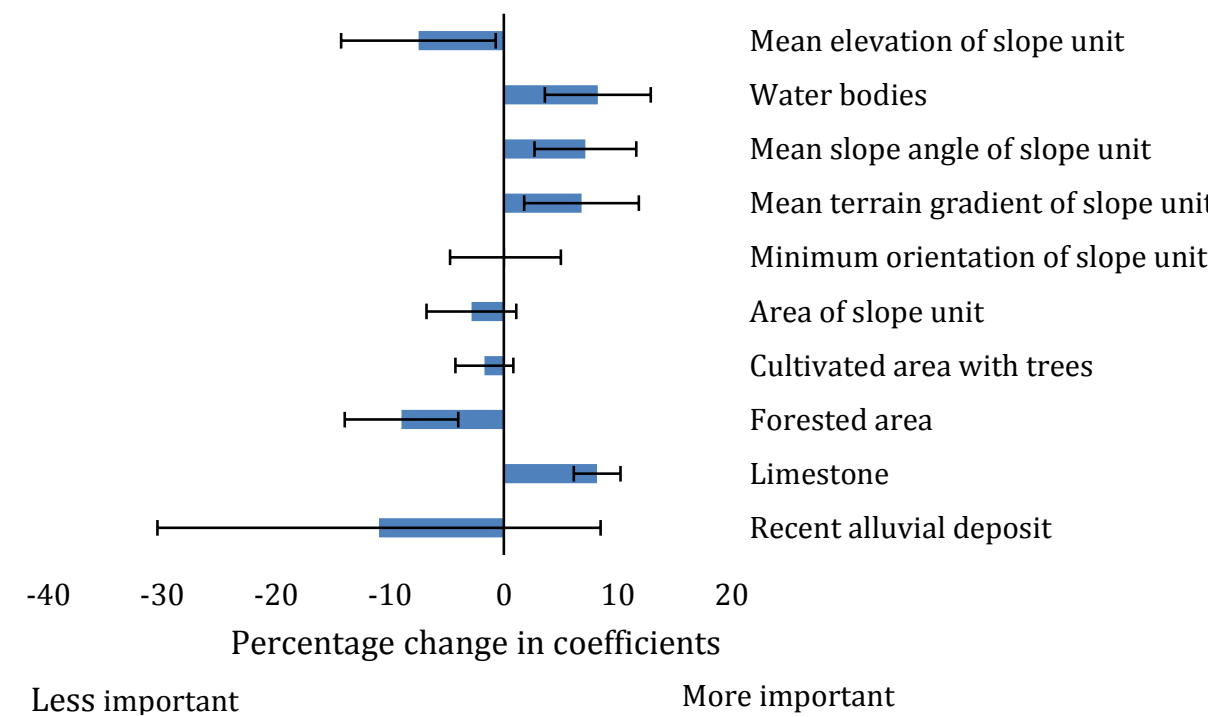

Figure 4.6. Percentage change in the coefficients with adding two landslide path dependency variables in sequential splitting. The changes of coefficients in variables that were 6 times or more common between two models were reported. Error bars represent standard error. 


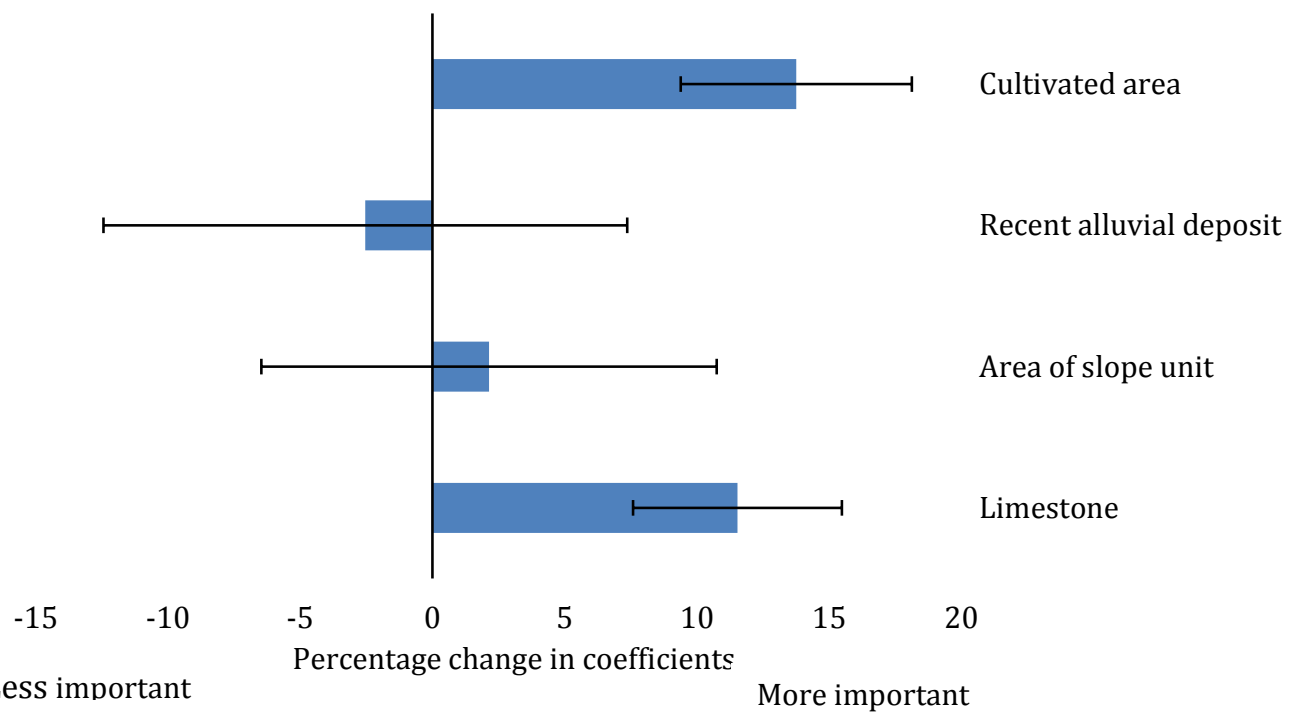

Figure 4.7. Percentage change in the coefficients with adding two landslide path dependency variables in non-sequential splitting. The changes of coefficients in variables that were 6 times or more common between two models were reported. Error bars represent standard error.

\subsubsection{Landslide susceptibility maps}

The landslide susceptibility maps for conventional and conventional plus path dependent in sequential and non-sequential splitting are presented in Figure 4.8. Note that in all cases the landslide susceptibility maps were made by averaging the probability of landslide occurrence in the slope units of all training and testing datasets. In both splitting approaches, there were no substantial differences between the histograms of probability of landslide occurrence between conventional and conventional plus path dependent landslide susceptibility (Figure $4.8 \mathrm{a}, \mathrm{b}, \mathrm{c}$ and $\mathrm{d}$ ). These slight differences between conventional and conventional plus path dependence susceptibility maps both in sequential and non-sequential splitting are in accordance with the slight differences in AUC values of their susceptibility models (Table 4.3). However, somewhat more slope units were predicted with probability of landslide occurrence lower than 0.2 , and less slope units with probability larger than 0.8 using conventional plus path dependent susceptibility than using conventional landslide susceptibility. 


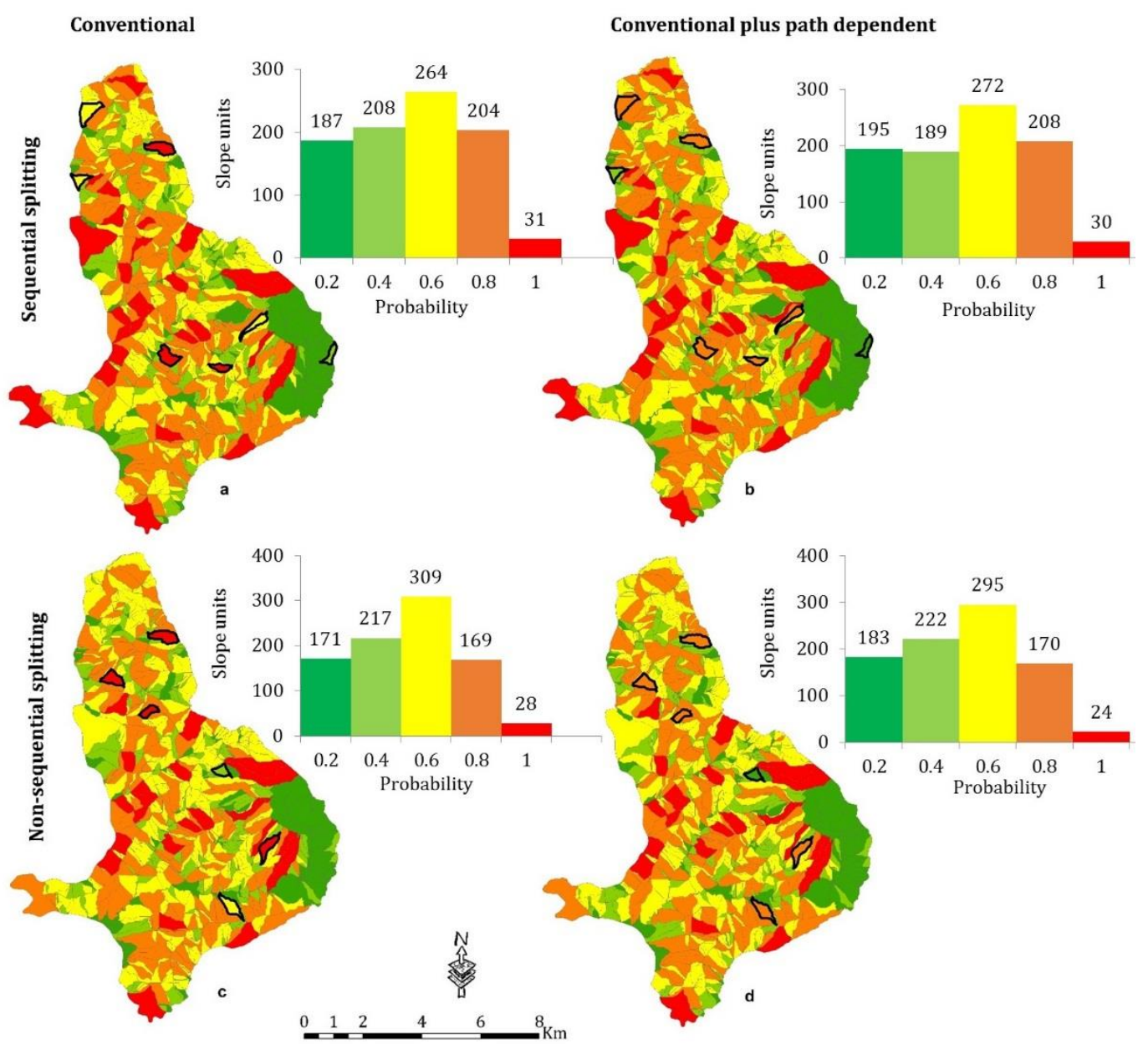

Figure 4.8. Conventional landslide susceptibility maps in sequential and non-sequential splitting $(\mathrm{a}, \mathrm{c})$ and conventional plus path dependent landslide susceptibility maps in sequential and non-sequential splitting $(b, d)$. Highlighted slope units in black indicate substantial differences in susceptibility between conventional and conventional plus path dependent landslide susceptibility maps.

The difference map for the sequential splitting, made by subtracting conventional susceptibility map from the conventional plus path dependent susceptibility map, shows that the susceptibility of slope units did not change substantially in about $46 \%$ of our study area (409 slope units) (Figure 4.9 a). In about $21 \%$ of the slope units, the probability of susceptibility slightly increased (192 slope units) and in $33 \%$ of the slope units (293 slope units) the probability of susceptibility slightly decreased. The difference map for non-sequential splitting showed similar results as well. 


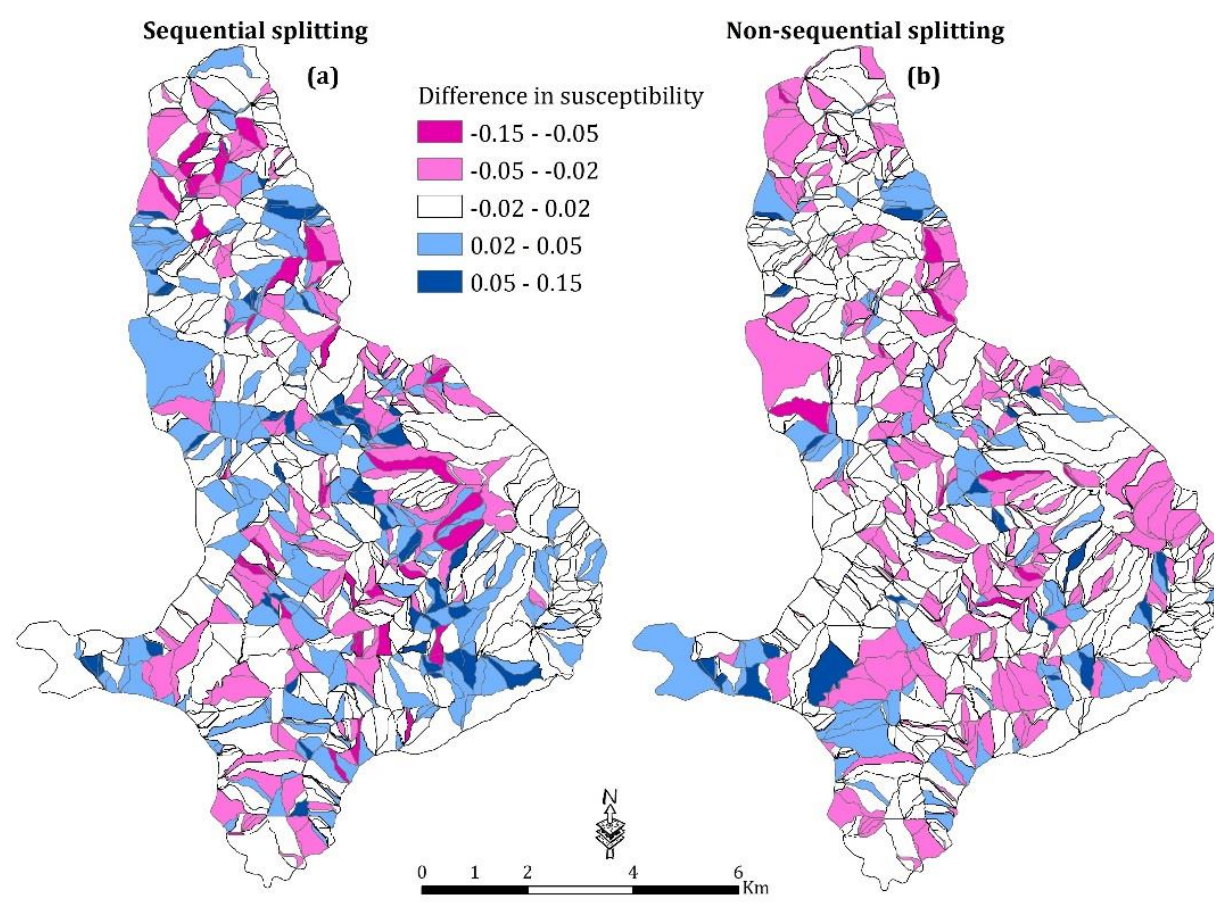

Figure 4.9. Difference between conventional landslide susceptibility map with conventional plus path dependent landslide susceptibility map in sequential (a) and non-sequential (b) splitting.

Path dependent landslide susceptibility maps in sequential and non-sequential splitting had substantial different geographical patterns in comparison to conventional and conventional plus path dependent landslide susceptibility maps (Figure 4.10a, b). The clear differences were in the slope units that did not have susceptibility smaller than 0.2 (Figure 4.10a) and larger than 0.8 (Figure 4.10a, b). This is due to the fact that susceptibility of the slope units to landslides decreases over time, which has been implemented into this model. 


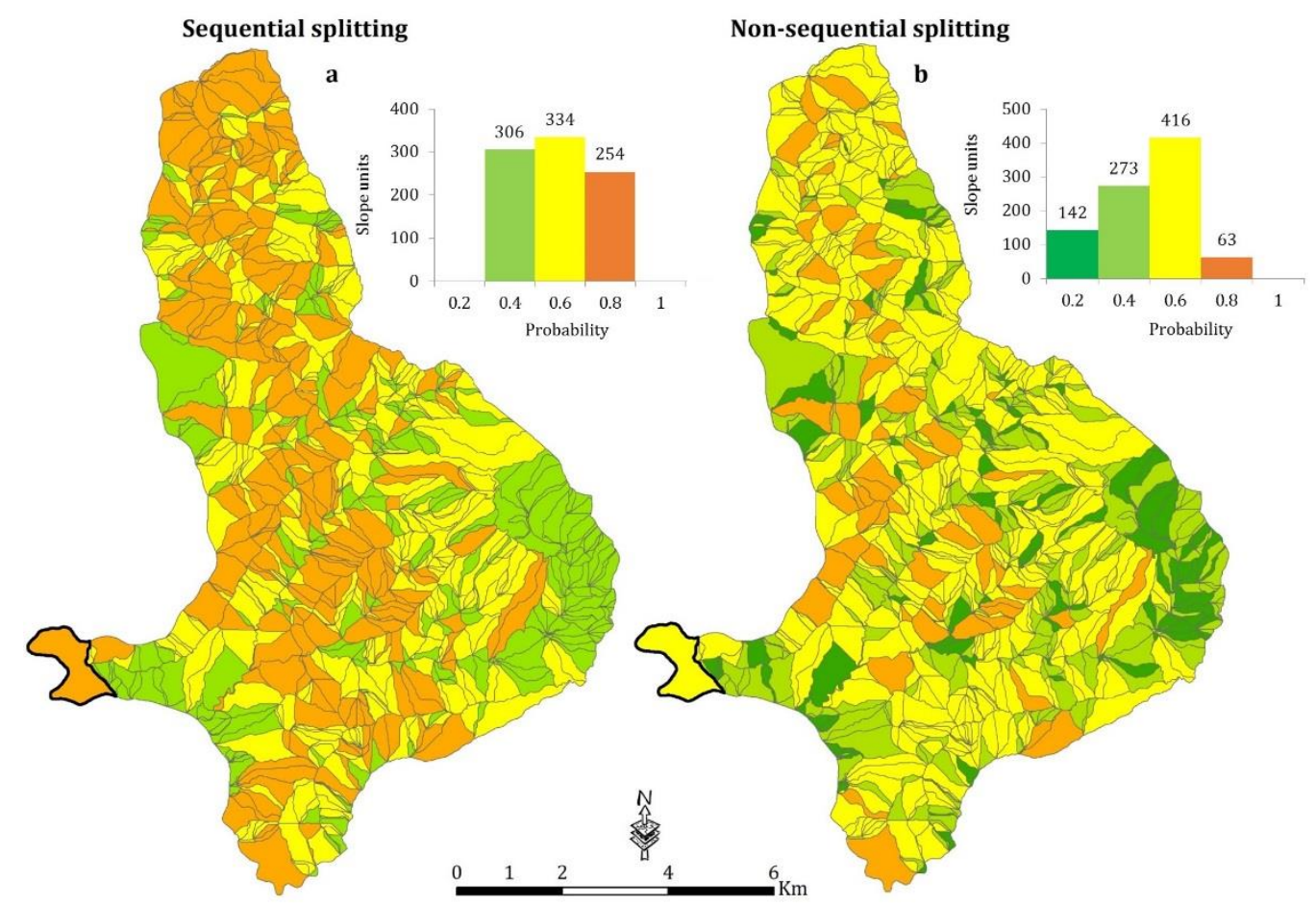

Figure 4.10. Path dependent landslide susceptibility maps predicted with only landslide path dependency variables in sequential splitting (a) and non-sequential splitting. The highlighted slope unit was selected as an example to show the change in susceptibility over time in Figure 4.12.

In sequential splitting, the difference map between conventional and path dependent landslide susceptibility maps showed that in $23 \%$ of the slope units, the susceptibility did not change, in about $23 \%$ of the slope units, the susceptibility increased, and in about $54 \%$ of the slope units, the susceptibility decreased (Figure 4.11a). In non-sequential splitting, in $21 \%$ of the slope units, the susceptibility did not change, in $11 \%$ of the slope units, the susceptibility increased, and in $68 \%$ of the slope units, the susceptibility decreased (Figure 4.11b). 


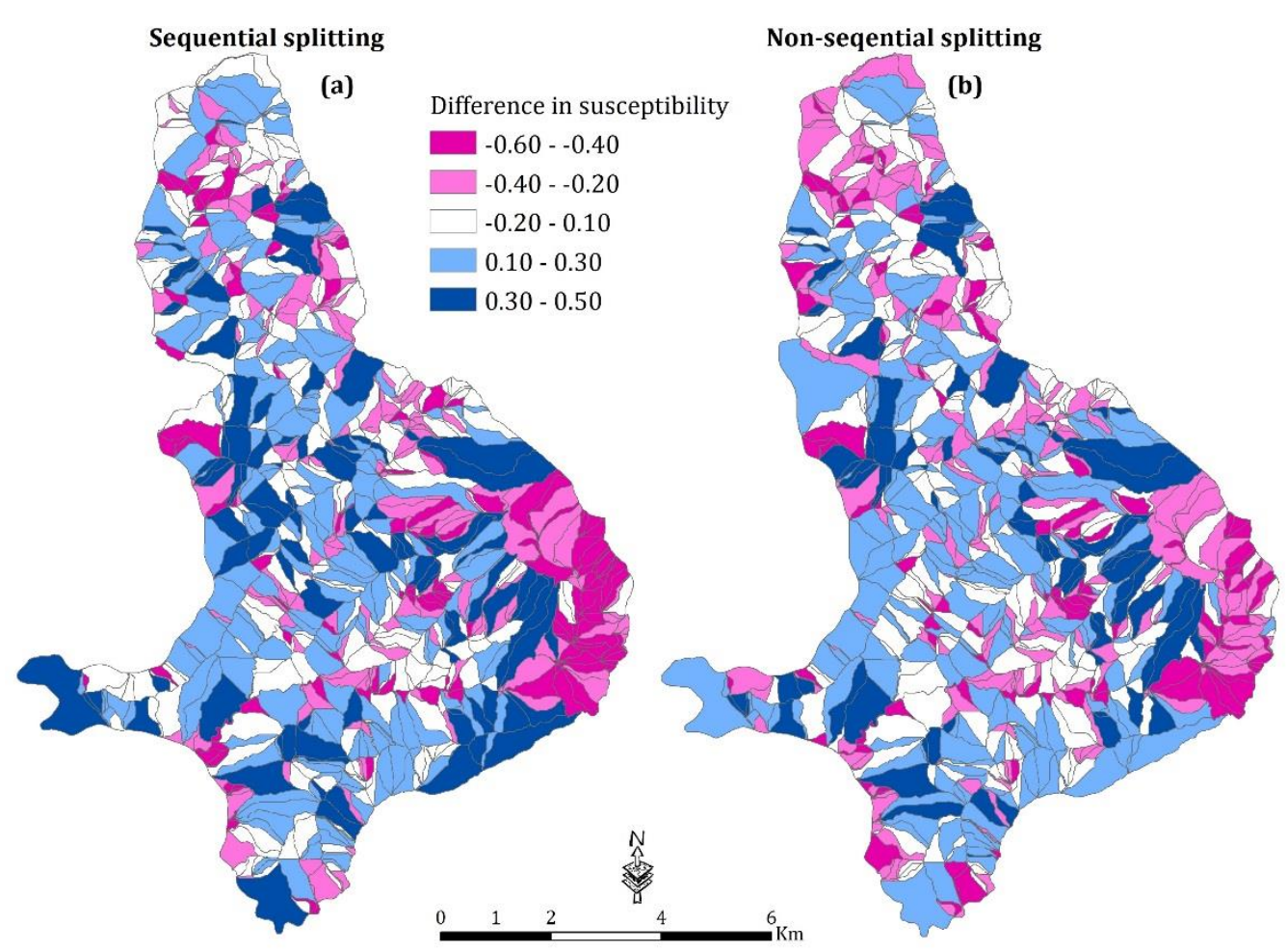

Figure 4.11. Difference between conventional landslide susceptibility map with path dependent landslide susceptibility in sequential (a) and non-sequential splitting (b).

\subsection{Discussion}

We will first discuss the concept of time-variant landslide susceptibility and then focus on the performance of the three landslide susceptibility models, and then discuss the importance of landslide path dependency variables in landslide susceptibility mapping. We will also discuss the possibility to create a simpler and easier to calculate landslide susceptibility model using DEM-derivative variables and landslide path dependency variables.

\subsubsection{Time-variant landslide susceptibility modelling}

By definition, conventional landslide susceptibility is considered to be constant over decadal timescales (Guzzetti et al. 1999; Guzzetti et al. 2005) (Figure 4.12). In conventional plus path dependent susceptibility, due to the effect of landslide path dependency, landslide susceptibility is dynamic and changes over the timescale of analysis. The fact that the change in the intensity of susceptibility is only slight, is due the fact that the set of 51 conditioning attributes already captures most of the spatio-temporal variation in landslide occurrence. This of course no longer the case 
in susceptibility maps prepared using the model with only the two landslide path dependency variables. Here, there is a quite intensive and dynamic change in the level of susceptibility, reflecting the imposed exponential decay of landslide path dependency (Samia et al. 2017a) (Figure 4.12).

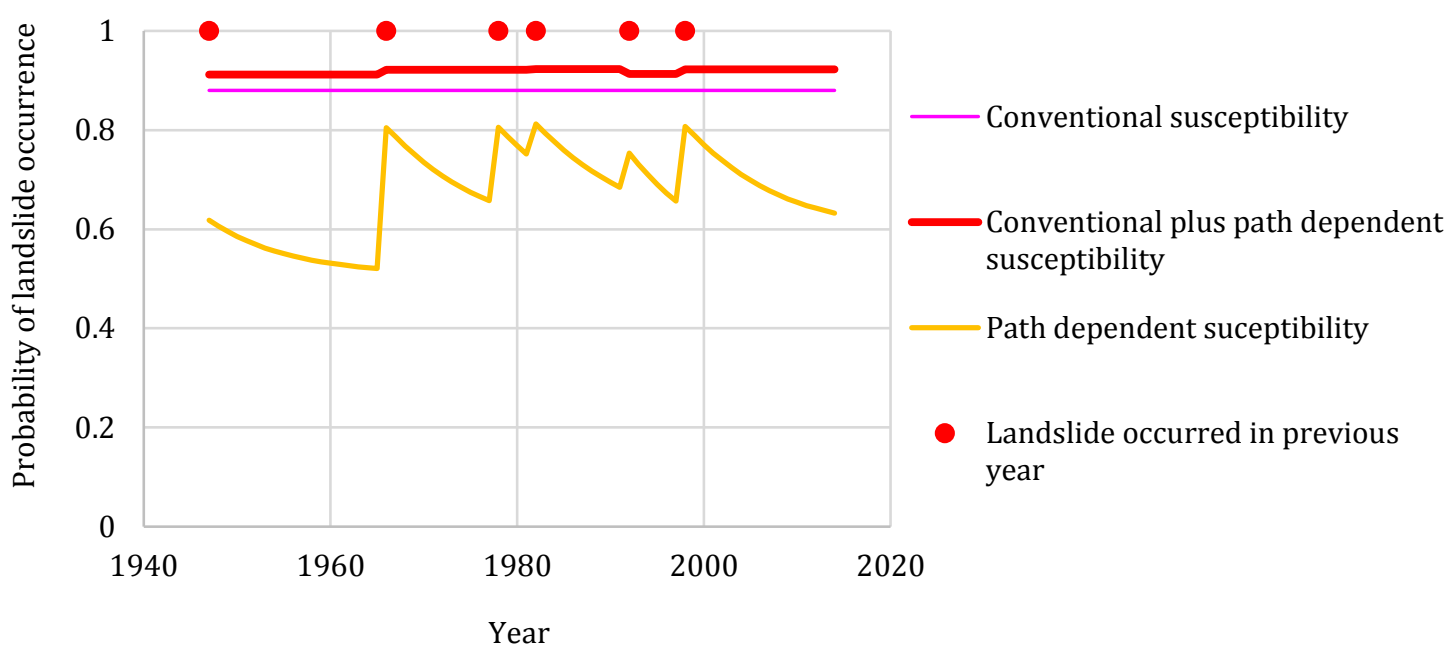

Figure 4.12. Modelled landslide susceptibility in the highlighted slope unit in Figure 4.10 using conventional, conventional plus path dependent and path dependent landslide susceptibility models and sequential splitting.

\subsubsection{Performance of landslide susceptibility models}

We found that adding landslide path dependency variables to the conditioning attributes variables used in conventional landslide susceptibility model slightly improved the performance of the susceptibility model (Table 4.1). These slight improvements in model performance are reflected in the high significance of landslide path dependency variables, affected the coefficients and significances of other variables (Figure 4.6, Figure 4.7), and inclusion and exclusion of other variables. The fact that the model improvement due to path dependent variables is nonetheless only slight, can be explained by: a) the 51 conventional variables (conditioning attributes) already capture almost all systematic variation; b) area under curve (AUC) considers the overall performance of the model without any spatial consideration while landslide path dependency attempts to describe the "local" effect on susceptibility and c) simply limited performance and relevance of landslide path dependency variables.

To explore the first of these three possibilities, we compared a landslide susceptibility model with only the two most significant conditioning attributes variables with path dependent landslide susceptibility model (with the two landslide path dependency variables). The performance of the conventional model was much closer to the performance of the path dependency model (Table 4.6, 
Table 4.3), indicating a relatively similar importance of landslide path dependency variables as the importance of conditioning attributes conventionally being used in landslide susceptibility modelling.

In an additional exploration, we found that a very simple landslide susceptibility model using only DEM-derived variables and landslide path dependency variables can lead to acceptable results (Table 4.6). This is potentially interesting since such landslide susceptibility models can be prepared easily, globally and within a short period of time. DEM-derived variables could easily be calculated for global extent with GIS related software, and high-resolution, accurate multi-temporal landslide inventories may be expected in the near future due to the recent widespread availability of high resolution remote sensing images. Therefore, landslide susceptibility model potentially could be prepared with a combination of DEMderived variables and landslide path dependency variables derived from multitemporal landslide inventory maps.

Table 4.6. landslide susceptibility models made by two most significant conditioning attributes variables and combination of DEM-derived variables with landslide path dependency variables.

\begin{tabular}{l|cc|cc}
\multirow{2}{*}{ AUC } & \multicolumn{2}{|l|}{$\begin{array}{l}\text { Landslide susceptibility model } \\
\text { (two most significant variables) }\end{array}$} & \multicolumn{2}{|c}{$\begin{array}{l}\text { Landslide susceptibility model } \\
\text { (DEM-derivatives plus path } \\
\text { dependent variables ) }\end{array}$} \\
\cline { 2 - 5 } & Sequential & Non-sequential & Sequential & Non-sequential \\
\hline Training & $0.712 \pm 0.010$ & $0.702 \pm 0.012$ & $0.749 \pm 0.009$ & $0.740 \pm 0.011$ \\
Testing & $0.700 \pm 0.012$ & $0.700 \pm 0.017$ & $0.725 \pm 0.013$ & $0.731 \pm 0.008$
\end{tabular}

Regarding the second point, performance of path dependence variables may have been limited by using the slope unit as mapping unit in the landslide susceptibility models. The values of landslide path dependency variables themselves were calculated based on geographical overlap with earlier landslides (Samia et al. 2017a). Since landslides are generally much smaller than the slope units, the landslide path dependency variables reflect a process that affects landsliding at smaller spatial scale than the scale at which they have now been used (slope units). The value of exponential decay $(b=-0.12)$ (Samia et al. 2017a) that was used was derived at the landslide scale, and may have needed to be re-estimated at the slope unit scale. This reasoning leaves open the possibility that for pixel-based susceptibility modelling the effect of landslide path dependency variables can be higher.

Regarding the two sampling strategies used in this work, the non-sequential splitting of the multi-temporal landslide inventory has the best performing in testing, and has also the smallest difference in the performance of the model between training and testing (Table 4.3, Table 4.6). We maintain that this is important, as it indicates we are less overfitting our susceptibility models. 


\subsubsection{Landslide path dependency as conditioning attributes in landslide susceptibility modelling}

We implemented our previously quantified landslide path dependency (Samia et al. 2017a) in landslide susceptibility modelling using only two landslide path dependency variables (Table 4.1). The landslide susceptibility predictions from these models (Figure 4.10a, b) performed acceptably with AUC $>0.660$ (Table 4.3) both for training and testing. This suggests that it is possible to use only landslide inventory itself to predict landslide susceptibility in areas where conditioning attributes are not available, or difficult to obtain. This could be potentially interesting since it has been always said that to do landslide susceptibility modelling, many conditioning attributes variables are needed (van Westen et al. 2003; Guzzetti et al. 2006a; Ghosh et al. 2011). We showed that this is not necessarily the case (Table 4.3, Figure 4.10a, b). For the calculation of landslide path dependency variables, time of landslide occurrence or mapping time of landslide, spatial association among landslides, geometric and topographic attributes of landslides were needed. Clearly, none of the landslide path dependency variables can be extracted from mono-temporal landslide inventory or from landslide inventories where landslides are mapped as points. This stresses the importance of monitoring of earlier landslides where multi-temporal landslides inventory can be provided and landslides are mapped with polygon (Samia et al. 2017b). Providing multi-temporal landslide inventory could be facilitated by remote sensing images and techniques. However, if there is no spatial association among landslides then the effect of landslide path dependency might be difficult to extract as we have only one test study site with multi-temporal landslide inventory. Besides that, if a landslide goes down slope and it is no longer present in the slope, then the effect of path dependency could be limited or even absent. For deep-seated landslides and earthflows, it has been speculated that reactivation is caused by a thin layer of smeared clays under the landslide body - the so-called bathtub effect (Baum and Reid 2000; Van Den Eeckhaut et al. 2007). If this effect is also behind some of the shallow landslides that we studied, then explanatory variables reflecting clay content and mineralogy may be useful to identify regions and hillslopes that are particularly susceptible to path dependency in landsliding.

\subsubsection{Data and method differences with previous landslide susceptibility modelling in the study area}

The previous landslide susceptibility modelling in our study area (Rossi et al, 2010) differs from ours in terms of data and the way of calculating the dependent variable. First, the multi-temporal landslide inventory has increased by four time slices (March and May 2010, April 2013 and April 2014) (Figure 4.1) using high resolution remote sensing images which have been used in this study, but were not available to Rossi et al. (2010). Besides, in our study landslides in the first available time slice (1939) were only used for computation of landslide path dependency variables , but were used as targets in landslide susceptibility modelling in Rossi et al. (2010). We calculated the dependent variable in slope units according to the presence or absence of each individual landslide. This allowed us to use the effect 
of each individual landslide purely in landslide susceptibility modelling while Rossi et al. (2010), combined all the landslides first, and then calculated the dependent variable with the presence or absence of all the combined landslides in slope units. These differences led to clear differences in the number of slope units located in different classes of probability to landslide occurrence. In Rossi et al (2010) more slope units (502 slope units) were predicted in the higher probability classes (0.6 0.8 and $0.8-1$ ), whereas we predicted less slope units (235 slope units in sequential splitting, and 197 slope units in non-sequential splitting) in the same high classes of probability (Figure 4.8a, c). Also, the number of slope units in the probability class of $0.4-0.6$ in our conventional landslide susceptibility maps are higher (264 slope units in sequential splitting, 309 slope units in non-sequential splitting) in comparison with 68 slope units predicted in this probability class by Rossi et al. (2010).

\subsubsection{Exportability of landslide path dependency variables in other areas for landslide susceptibility modelling}

Where multi-temporal landslide inventories are available (to our knowledge the only large multi-temporal landslide inventory available worldwide is the multitemporal landslide inventory used in this work), and the geological, climate conditions and type of landslides are similar to our study area, our exponential decay coefficient $b=-0.12$ from susceptibility temporal decay (Table 4.1 and eq. 1 ), and spatial probability of each landslide causing follow-up landslide could directly be used to model susceptibility to landslide. However, most landslide inventories are mono-temporal usually recorded after extreme external triggers (e.g., rainfall and earthquake). From such landslide inventories, the exponential decay coefficient from susceptibility temporal decay cannot be computed. However, from geometric attributes (e.g., size, shape) and topographic attributes of each landslide in the mono-temporal landslide inventory, the spatial probability of earlier landslides causing follow-up landslides (Table 4.1) is computable if we assume that the model to predict the occurrence of follow-up landslides by Samia et al. (2017a) is valid. In this context, for every known landslide we would have a probability that shows whether a landslide will have a follow-up landslide, or not. This landslide path dependency variable in our study area was found to be the most significant and selected variable by the logistic regression when using it individually, or combined with conditioning attributes variables in landslide susceptibility modelling (Table 4.1). Therefore, a combination of path dependent variables with a range of other possibility conditioning attributes or DEM-derived variables could be used to model susceptibility to landslide.

\subsection{Conclusions}

For our study area, where adding landslide path dependency variables to the conditioning attributes conventionally used in landslide susceptibility modelling improves the performance of landslide susceptibility model slightly. However, the 
resulting landslide susceptibility maps from conventional and conventional plus path dependent landslide susceptibility models are not substantially different. The highest performance for landslide susceptibility model was obtained in conventional plus path dependent landslide susceptibility model with the AUC = $0.775 \pm 0.006$ in sequential splitting and AUC $=0.754 \pm 0.012$ in non-sequential splitting. In addition, a landslide susceptibility model purely made by two landslide path dependency variables has decent model performance with the AUC values > 0.660 in sequential and non-sequential splitting. The non-sequential sampling strategy has a better model performance in the testing dataset, and less difference with the performance of the model between training and testing datasets. Moreover, the spatial probability of earlier landslide causing follow-up landslide was selected in 18 out of 20 runs logistic regression for landslide susceptibility modelling. The susceptibility temporal decay was also selected in $60 \%$ of the runs (12 out of 20) of logistic regression. These landslide path dependency variables also changed the significance, inclusion and exclusion of other variables selected by logistic regression. A simple, easily computed landslide susceptibility model with reliable model performance can be obtained using combination of DEM-derived variables and landslide path dependency variables. Our landslide path dependency variables can possibly be applied to model susceptibility to landslide in areas similar to our study area where multi-temporal landslide inventory or monotemporal landslide inventory are available.

Acknowledgements: This work is part of J Samia PhD project at laboratory of GeoInformation Science and Remote Sensing and Soil Geography and Landscape groups of Wageningen University and Research financed by Ministry of Science, Research and Technology of Iran. We would like to thank the two anonymous reviewers for their constructive comments on the preparation of the final manuscript. 


\section{Chapter 5}

\section{Pixel-based dynamic path dependent landslide susceptibility modelling}

This chapter is based on:

Samia, J., Temme, A., Bregt, A., Wallinga, J., Guzzetti, F., Ardizonne, F. Pixel-based dynamic path dependent landslide susceptibility modelling. Manuscript to be submitted to Natural Hazards and Earth System Sciences (NHESS). 


\begin{abstract}
A pixel-based conventional landslide susceptibility model was compared with a model that includes landslide path dependency and also with a purely path dependent landslide susceptibility model. To quantify path dependency among landslides, we used a space-time clustering measure derived from Ripley's spacetime K Function implemented on a point-based multi-temporal landslide inventory from the Collazzone study area in central Italy. We found that the space-time clustering measure remained high within a decadal time scale and within $200 \mathrm{~m}$ distance from previous landslide with an exponential decay over these spatial and temporal scales. The maximum and the sum of space-time clustering measures for every pixel were computed as landslide path dependency variables and used along with DEM-derivative variables in landslide susceptibility modelling. Landslide path dependency variables modelled landslide susceptibility with a reasonable accuracy with AUC of 0.720 , better than conventional landslide susceptibility modelled with DEM-derivative variables with AUC of 0.672. Combined landslide path dependency variables with DEM-derivatives in conventional plus path dependent landslide susceptibility model had the best model performance and accuracy with AUC of 0.749 . The conventional plus path dependent and path dependent landslide susceptibility maps are time-variant and change over time unlike conventional landslide susceptibility map which is constant overtime. The time-variant path dependent landslide susceptibility maps allow modifications in landslide hazard and risk assessment.
\end{abstract}




\subsection{Introduction}

Landslides as a hydro-geomorphological type of natural disaster have destructive effects on the environment, infrastructure and society. Landslide susceptibility modelling calculates the likelihood of landslide occurrence in a certain location (Brabb 1984). The resulting landslide susceptibility maps which show where landslides are likely to occur (Guzzetti et al. 2005), are key tools for the preparation and mitigation of the destructive effects of landslides. Different methods and techniques have been used for landslide susceptibility modelling. Reichenbach et al. (2018) classified all these methods and techniques into five groups: (i) direct geomorphological mapping, (ii) analysis of landslide inventories, (iii) heuristic or index-based approaches, (iv) physically or process-based methods, and (v) statistical-based techniques.

Statistically-based landslide susceptibility techniques have been the preferred technique in landslide susceptibility modelling (Reichenbach et al. 2018). In statistical landslide susceptibility modelling, relations are explored between landslide inventory maps and a set of conditioning attributes (e.g., slope and geology) (Van Westen et al. 2003; Guzzetti et al. 2005). Landslide inventory maps that document the distribution and type of landslides, are therefore a crucial input for landslide susceptibility modelling (Van Westen et al. 2008; Guzzetti et al. 2012). Direct field mapping, aerial photographs and other remote sensing images are the main sources for such mapping of landslides (Guzzetti et al. 2012). Traditionally landslides in inventory maps were stored in a point format, with points representing the geographic location of landslide center points. More recently, leveraging better technology such as GIS, landslide inventory maps have become typically polygon-based, with polygons representing landslide outlines (Xu 2015). Conditioning attributes are usaully DEM-derivatives (Digital Elevation Model) along with geological, soil and land use data (Neuhäuser et al. 2012; Günther et al. 2014; Reichenbach et al. 2018). DEM-derivatives are nowadays easily computed at fine resolutions $(\leq 30 \mathrm{~m})$ for all locations in the world in various open source and commercial software packages whereas the geology, land use and soil data are not always available in similar detail. Hence, the minimum available data for landslide susceptibility modelling are point-based landslide inventory with DEM-derived conditioning attributes.

Recently, we proposed the concept of time-variant landslide susceptibility in which the space-time history of landslides is a component of landslide susceptibility (Samia et al. 2017a; Samia et al. 2017b). We referred to this as path dependency, a term adopted from complex system theory where it is used to describe the concept that the history of a system partly determines its future state (Phillips 2006). In our study area in Italy (Figure 5.1), we found the existence of path dependency among landslides: earlier landslides locally increase the susceptibility for future landslides for about two decades during which the susceptibility decays exponentially back to previous levels (Samia et al. 2017a). We implemented the effect of landslide path dependency in landslide susceptibility modelling at hillslope unit scale, and investigated the impact on landslide susceptibility models and maps. These models 
and maps differed only slightly from conventional landslide susceptibility models (Samia et al. 2018). We argued that the limited impact of landslide path dependency on model predictions was due to the fact that landslide path dependency affects landslide patterns at spatial scales smaller than hillslope units, and it was hypothesized that differences between models were likely to increase when including path dependency at pixel scales.

Our path dependent landslide susceptibility model was based on a unique polygonbased multi-temporal landslide inventory (Samia et al. 2017a). As such multitemporal inventories are usually not available for most landslide prone areas in the world, the applicability of our previously explored method is limited. In most places, another approach would therefore be needed to implement landslide path dependency in landslide susceptibly modelling. Research is needed to leverage landslide path dependency effects in such areas. Admittedly, multi-temporal polygon-based landslide inventories are not currently available, but multi-temporal point-based landslide inventories are more often available and can be prepared easier than polygon-based multi-temporal landslide inventory.

The objective of this work is thus to quantify landslide path dependency in a multitemporal point-based landslide inventory, and then consider it in landslide susceptibility modelling at the resolution of $10 \mathrm{~m}$ pixels. We hypothesize that including landslide path dependency will improve the performance of conventional landslide susceptibility models. We also present a purely path dependent landslide susceptibility model and compare its results with conventional landslide susceptibility modelling. We again use the unique multi-temporal landslide inventory from the Collazzone study area (Figure 5.1) (Guzzetti et al. 2006a; Ardizzone et al. 2007; Ardizzone et al. 2013).

\subsection{Study area and data}

The Collazzone study area in Umbria region in central Italy (Figure 5.1) has been affected by landslides in many years. The study area extends about $80 \mathrm{~km} 2$ with elevation between 145 to $634 \mathrm{~m}$ above sea level and slope between 0 to $64^{\circ}$ derived from a 10 by 10 meter Digital Train Model (DTM). The DTM was prepared using interpolation of contour lines of 5 and 10 meters in the 1:10,000 topographical map (Guzzetti et al. 2006b). Landslides are abundant in this area, and range from recent shallow landslides to old deep-seated landslides (Guzzetti et al. 2006a). Intense and prolonged rainfall and rapid snowmelt are the main triggers for landslide occurrence in this area (Cardinali et al. 2000; Ardizzone et al. 2007). A rich and detailed multi-temporal landslide inventory has been mapped continuously in 19 different time slices containing 3391 landslides. The age of landslides ranges from relict and very old landslides until landslides that occurred in 2014. For preparation of the multi-temporal landslide inventory, aerial photographs, direct field mapping after major rainfall storms and snowmelt, and remote sensing images were used (Guzzetti et al. 2006a; Galli et al. 2008; Ardizzone et al. 2013). The age of landslides 
in the first two time slices of the multi-temporal inventory is not accurately known. Therefore, these time slices were not used in this work. The time slice of 1939 was only used in computation of landslide path dependency variables and not in the landslide susceptibility modelling because of its unknown past. Therefore, a multitemporal landslide inventory was used that contains 16 time slices of mapped landslides from 1947 to landslides in 2014 (Figure 5.1). A full description of the study area and the multi-temporal landslide inventory can be found in (Guzzetti et al. 2006a; Ardizzone et al. 2007; Galli et al. 2008; Guzzetti et al. 2009; Samia et al. 2017b).

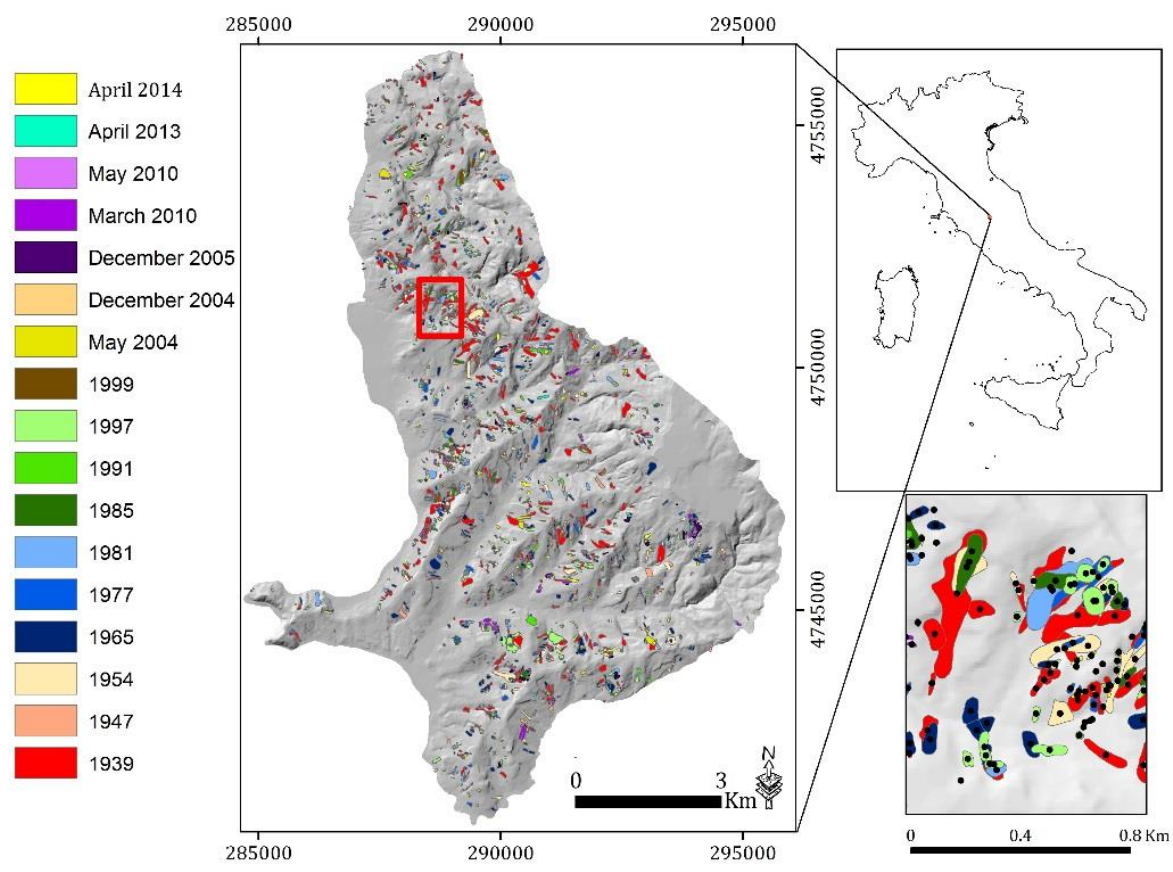

Figure 5.1. Multi-temporal landslide inventory overlaying a shaded relief image (left map) (adapted from (Samia et al. 2017b)). Location of Umbria region and of the Collazzone study area (right upper map). The coordinate system of maps is EPSG:32633 (www.spatialreference.org). Landslide points were constructed in the geometric centre of each landslide polygon (map in the right lower corner). The rectangle red extent shows the location of the map in the lower right. 

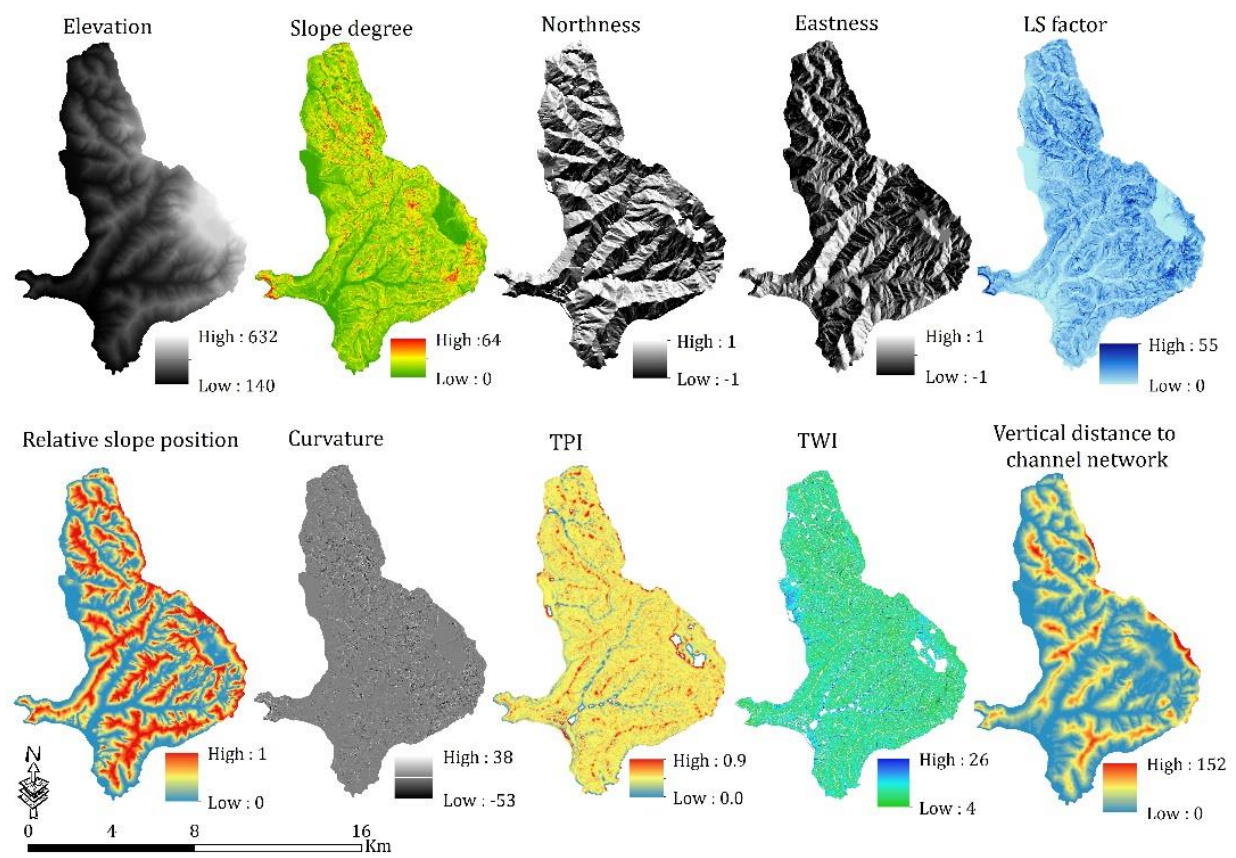

Figure 5.2. DEM and nine derivatives used in conventional landslide susceptibility modelling.

\subsection{Methods}

We used logistic regression at pixel resolution to construct three landslide susceptibility models: (i) a conventional landslide susceptibility model, (ii) a conventional plus path dependent landslide susceptibility model and (iii) a purely path dependent landslide susceptibility model (Samia et al, 2018). We compared the performance of these models using Area Under Curve (AUC) values from the Receiver Operating Characteristic (ROC) (Mason and Graham 2002), and selected the optimal model using the Akaike Information Criterion (AIC) (Akaike 1998), which penalizes the use of additional variables in a model. Finally, the coefficients of the variables selected by the landslide susceptibility models and the resulting landslide susceptibility maps were compared. 


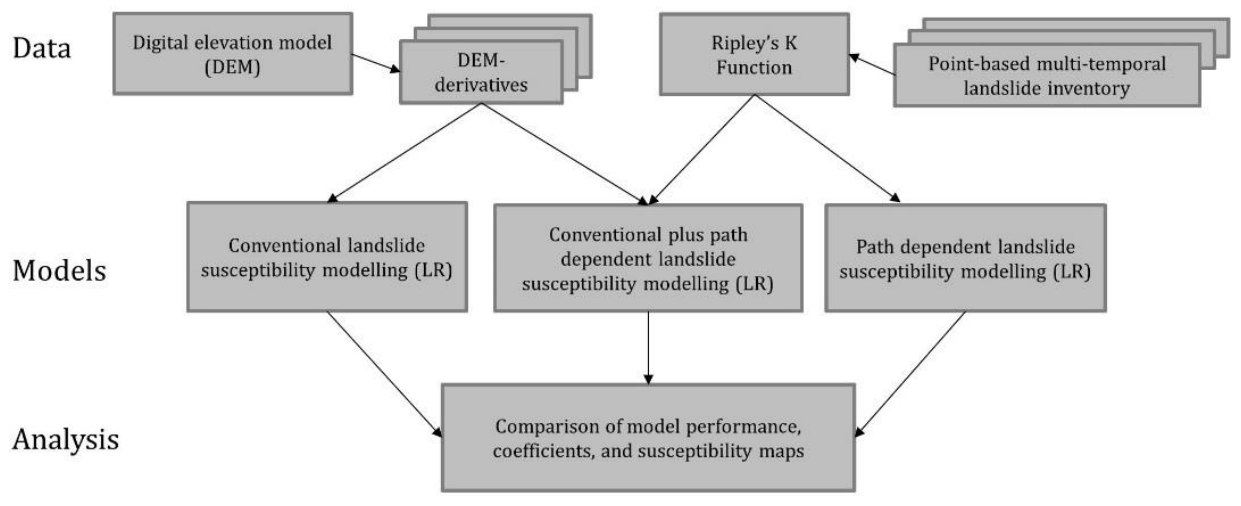

Figure 5.3. Flowchart of methods.

\subsubsection{Quantifying landslide path dependency using Ripley's space- time K function}

We recently characterized and quantified the existence of spatial-temporal path dependency among landslides for the Collazzone study area. The landslide path dependency exhibited an exponential decay where landslide susceptibility increases immediately after a landslide and decreases to the background susceptibility in about two decades (Samia et al. 2017a). The addition of landslide path dependency to a slope unit-based landslide susceptibility model did not substantially improve in the performance of the landslide susceptibility model (Samia et al. 2018).

Our previous quantification required information about the space-time overlap among landslide polygons (Samia et al. 2017b; Samia et al. 2017a). The novel aspect of the present paper is that we instead quantify landslide path dependency among centre points of landslides rather than polygons of landslides. For this quantification, we use Ripley's K Function (Ripley 1976; Diggle et al. 1995). Ripley's $\mathrm{K}$ function - which has been used mainly in purely spatial point pattern analysis reflects the degree of spatial clustering of certain events (e.g., landslides (Tonini et al. 2014), forest fire (Gavin et al. 2006) , crimes (Levine 2006) and disease outbreak (Hinman et al. 2006)). The function determines whether events are clustered, dispersed of randomly distributed in the area of interest. In a landslide path dependency context, Ripley's $\mathrm{K}$ function is now used in space-time, reflecting the degree to which landslides occur clustered, dispersed or randomly distributed in space-time.

Ripley's space-time K function tests whether the number of events that is observed in a space-time cylinder around an initial event is equal to what is expected given the average point density in space and time (Ripley 1976; Ripley 1977; Diggle et al. 1995). The space-time cylinder I (h, $\Delta$ ) is defined as: 
$I_{(h, \Delta)}\left(d_{i j}, t_{i j}\right)=\left\{\begin{array}{c}1,\left(d_{i j} \leq h \text { and }\left(t_{i j} \leq \Delta\right)\right) \\ 0, \text { otherwise }\end{array}\right.$

where $\mathrm{h}$ shows the distance increment, $\Delta$ shows the time increment, $\mathrm{i}$ and $\mathrm{j}$ are landslide points, with $\mathrm{d}$ and $\mathrm{t}$ reflecting the distance and time between these points respectively.

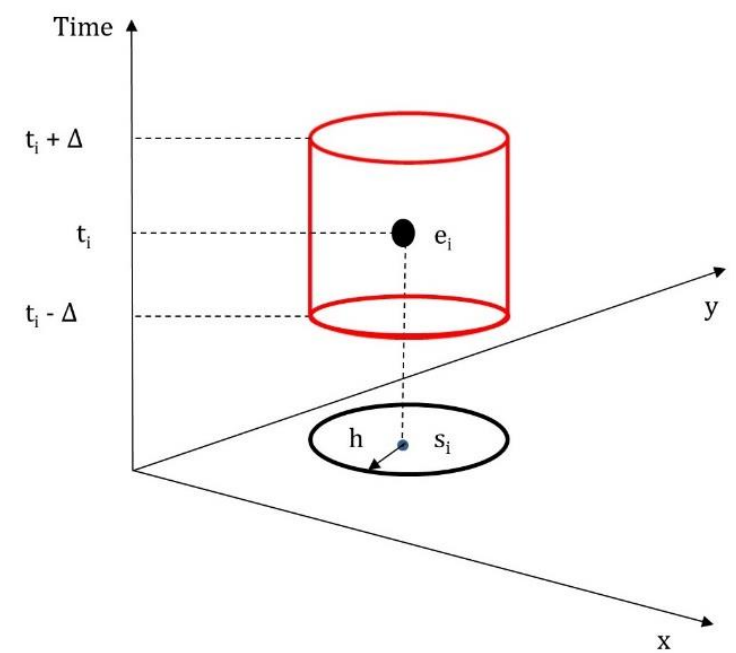

Figure 5.4. Space-time cylinder neighbourhood (Smith 2016) for a landslide event ( $\left.\mathrm{e}_{\mathrm{i}}\right)$.

Ripley's $\mathrm{K}$ function for one space-time cylinder of $\mathrm{h}$ and $\Delta$ is defined as:

$K(h, \Delta)=\frac{1}{\lambda_{s t}} \sum_{j \neq i} E\left[I_{(h, \Delta)}\left(d_{i j}, t_{i j}\right)\right]$

where $\lambda_{s t}$ reflects the space-time intensity of the landslides (i.e., the expected number of landslides per unit of space-time volume), which is calculated as:

$\lambda_{s t}=\frac{n}{a \cdot(R) \times\left(t_{\max }-t_{\min }\right)}$

where $\mathrm{n}$ is the number of landslides in the entire inventory, $\mathrm{t}$ shows time, and a.(R) reflects the size of the area.

Therefore, the expected Ripley's space-time K function for all space-time cylinders around all landslides is defined as:

$K(h, \Delta)=\frac{1}{n . \lambda_{s t}} \sum_{i=1}^{n} \sum_{j \neq i} E\left[I_{(h, \Delta)}\left(d_{i j}, t_{i j}\right)\right]$ 
Similarly, the observed Ripley's space-time $\mathrm{K}$ function is calculated from the landslide inventory as:

$\widehat{K}(h, \Delta)=\frac{1}{n \cdot \hat{\lambda}_{s t}} \sum_{i=1}^{n} \sum_{j \neq i} I_{(h, \Delta)}\left(d_{i j}, t_{i j}\right)$

To quantify how strong is that the expected landslides to be at a certain distance ti,j and di,j of the previous landslides, the space-time clustering measure was calculated as following:

Space - time clustering measure $=\frac{\widehat{K}(h, \Delta)}{K(h, \Delta)}$

The space-time clustering measure shows how more likely is that a landslide at a certain time and space distance from the previous landslides occur. Space-time clustering measure values $>1$ indicate clustering and values $<1$ indicate dispersion.

We calculated the space-time clustering measure for w wide range of $h, \Delta$, and then fitted an exponential function of $\mathrm{h}$ and $\Delta$ to the results. Then, using this exponential function, for every pixel in each time slice of the multi-temporal landslide inventory, we calculated the space-time clustering measure relative to the all pixels depending on when a landslide last time occurred closely to every pixel. Then, we calculated two landslide path dependency variables. The first variable reflects the maximum value of all space-time clustering measures for all previous landslides near a pixel. The second variable is the sum of all space-time clustering measures of all previous landslides near a pixel.

\subsubsection{Logistic regression}

Logistic regression is the most frequently used statistical model in landslide susceptibility modelling (Reichenbach et al. 2018). In landslide susceptibility modelling, relations between presence and absence of landslides as binary target (dependent) variable are explained by a set of explanatory (independent) variables such as slope, aspect and geology. The statistics and coefficients of the relations are used to classify the area of interest to different intensity of susceptibility to landslides. In this paper, DEM-derivatives that were used as explanatory variables are slope angle, elevation, curvature, northness, eastness, topographic position index (TPI), topographic wetness index (TWI), LS factor, vertical distance to channel network and relative slope position (Figure 2). Also, the two landslide path dependency variables computed by space-time Ripley's K Function (see section 5.3.1) were used as extra explanatory variables. The binary target variable is the presence and absence of landslides from the multi-temporal landslide inventory.

\subsubsection{Landslide susceptibility modelling}

We performed conventional, conventional plus path dependent and purely path dependent landslide susceptibility modelling using logistic regression. 
Conventional landslide susceptibility modelling was performed using only the DEM-derivatives as conditioning attributes. In conventional plus path dependent landslide susceptibility modelling, DEM-derivatives (Figure 5.2) along with the two landslide path dependency variables (see section 5.3.1) were used. For purely path dependent landslide susceptibility modelling only the two landslide path dependency variables were used. We divided the multi-temporal landslide inventory into training and testing datasets based on the non-sequential splitting sampling strategy (Samia et al. 2018). The non-sequential selection of time slices from the multi-temporal landslide inventory prioritizes an equal range of temporal separations between time slices in the training and the testing datasets. For the training dataset, the $1^{\text {st }}, 2^{\text {nd }}, 5^{\text {th }}, 6^{\text {th }}, 9^{\text {th }}, 10^{\text {th }}, 13^{\text {th }}$ and $14^{\text {th }}$ time slices from the multitemporal landslide inventory (Figure 5.1) were used (covering a period of 63 years), and for the testing dataset, the $3^{\text {rd }}, 4^{\text {th }}, 7^{\text {th }}, 8^{\text {th }}, 11^{\text {th }}, 12^{\text {th }}, 15^{\text {th }}$ and $16^{\text {th }}$ time slices were used (covering a period of 49 years). Note that the 1 st time slice in the training dataset starts from 1947 - that is because the first time slice in the inventory, from 1939, has no known landslide history. The number of pixels with landslides was smaller than the number of pixels without landslides in both training and testing datasets. Therefore, we randomly selected 5000 pixels with landslides and 5000 pixels without landslides to create equal datasets both for training and testing. This random selection of pixels was repeated 10 times both in the training and testing datasets. After preparation of the 10 training datasets, logistic regression was applied to these 10 training datasets. We imposed an entry probability of 0.05 and a removal probability of 0.06 to reduce the risk of overfitting in the model. To avoid multicollinearity, we allowed only inter-variable correlations less than 0.6. The performance of models was assessed using AUC and AIC values. To test the models, the 10 training models were applied to the 10 independent testing datasets, and the AUC values were computed again. The coefficients of the models with highest performance in terms of AUC values, were used to map susceptibility to landslides. Finally, we compared the landslide susceptibility maps resulting from conventional, conventional plus path dependent and purely path dependent susceptibility.

\subsection{Results}

\subsubsection{Spatial-temporal dynamic of landslide path dependency}

The space-time Ripley's K Function confirmed the existence of path dependency (clustering) among landslides at small spatial and temporal distance. Clustering decreased exponentially in space and time (Figure 5.5). The space-time clustering measure (eq 6) is high in the space-time vicinity of an earlier landslide. Apparently landslide susceptibility increases immediately after occurrence of an earlier landslide. The effect remains for a time period of about two decades and a spatial distance of about 200 meter. Within this spatial and temporal scales, follow-up landslides (Samia et al,2017a, 2017b) possibly occur, and beyond that, the intensity of susceptibility decays exponentially. 


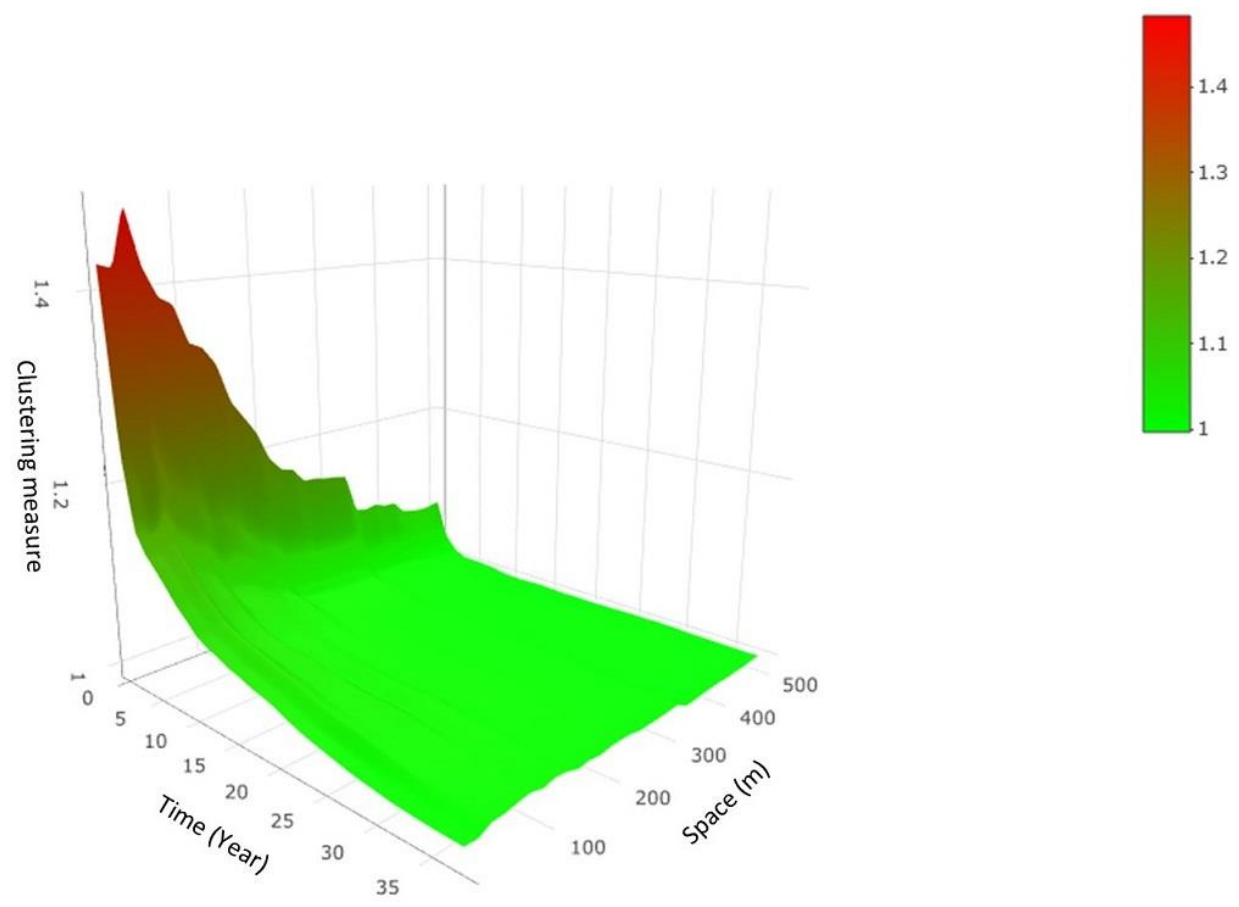

Figure 5.5. Space-time dynamic of landslide path dependency.

The exponential decay function that was fitted to the space-time clustering data is:

Clustering measure $(s, t)=$ clustering measure ${ }_{a} \exp ^{(-0.06 t)} * \exp ^{(-0.017 d)}$

This function shows that the space-time clustering measure decays over temporal scale of $b\left(\right.$ year $\left.{ }^{-1}\right)$ and spatial scale of $d\left(\right.$ meter $\left.^{-1}\right)$. The values of exponential coefficients $\left(b=-0.06 \pm 0.001\right.$ year $^{-1}$ and $d=-0.017 \pm 0.004$ meter $\left.^{-1}\right)$ indicate that space-time cluster measure values decrease quickly with the increment in the space and time scales from an earlier landslide. The residual standard error of the exponential function is $0.01(-)$, which is satisfactory.

\subsubsection{Model performance}

The performance of landslide susceptibility model was highest in conventional plus path-dependency model, both when expressed as AUC values, and when expressed as AIC values (Table 5.1). Even the purely path dependent landslide susceptibility model constructed with only two landslide path dependency variables performed better than the conventional landslide susceptibility model. The conventional landslide susceptibility model constructed with DEM-derivatives has the lowest model performance in the training and testing datasets. 
Table 5.1. Area Under Curve (AUC) and Akaike Information Criterion (AIC) values in conventional, conventional plus path dependent and path dependent landslide susceptibility modelling.

\begin{tabular}{llll}
\hline $\begin{array}{l}\text { AUC and AIC } \\
\text { values }\end{array}$ & $\begin{array}{l}\text { Conventional } \\
\text { susceptibility } \\
\text { model }\end{array}$ & $\begin{array}{l}\text { Conventional plus path } \\
\text { dependent susceptibility } \\
\text { model }\end{array}$ & $\begin{array}{l}\text { Path dependent } \\
\text { susceptibility } \\
\text { model }\end{array}$ \\
\hline AUC training & $0.672 \pm 0.006$ & $0.749 \pm 0.002$ & $0.721 \pm 0.004$ \\
AIC training & $13065 \pm 69$ & $12000 \pm 94$ & $12469 \pm 62$ \\
AUC testing & $0.656 \pm 0.005$ & $0.718 \pm 0.003$ & $0.698 \pm 0.004$ \\
\hline
\end{tabular}

DEM-derivatives in conventional and conventional plus path dependent susceptibility and the two landslide path dependency variables in conventional plus path dependent and purely path dependent susceptibility were always significant $(p<0.05)$. These variables were selected in all 10 training datasets in conventional, conventional plus path dependent and path dependent landslide susceptibility modelling.

In both 10 training and 10 testing datasets, the contingency tables showed that conventional landslide susceptibility model has substantial difference with conventional plus path dependent and path dependent landslide susceptibility models. The false positive (pixels without landslide but predicted with landslides) in both training and testing datasets in conventional susceptibility is more than conventional plus path dependent and path dependent susceptibility models. The true negative (pixels without landslides and predicted without landslides) is less in conventional than conventional plus path dependent and path dependent susceptibility models. The variation in the differences is larger in the training datasets than the testing datasets. 
Table 5.2. Contingency tables for conventional, conventional plus path dependent and path dependent landslide susceptibility modelling with highlighted differences.

\begin{tabular}{|c|c|c|c|c|c|c|c|}
\hline & & \multicolumn{2}{|c|}{$\begin{array}{c}\text { Conventional susceptibility } \\
\text { model }\end{array}$} & \multicolumn{2}{|c|}{$\begin{array}{c}\text { Conventional plus path } \\
\text { dependent susceptibility model }\end{array}$} & \multicolumn{2}{|c|}{$\begin{array}{c}\text { Path dependent } \\
\text { susceptibility model }\end{array}$} \\
\hline & & Observ & ndslides & Observ & slides & Observe & ndslides \\
\hline \multirow{2}{*}{$\begin{array}{l}\text { Predicted } \\
\text { landslides in } \\
\text { training }\end{array}$} & Yes & $\begin{array}{c}\text { YES } \\
32 \pm 0.37\end{array}$ & $\begin{array}{c}\text { NO } \\
20 \pm 0.67\end{array}$ & $\begin{array}{c}\text { YES } \\
33 \pm 0.29\end{array}$ & $\begin{array}{c}\text { NO } \\
14 \pm 0.15\end{array}$ & $\begin{array}{c}\text { YES } \\
31 \pm 0.8\end{array}$ & $\begin{array}{c}\text { NO } \\
13 \pm 0.32\end{array}$ \\
\hline & No & $18 \pm 0.37$ & $30 \pm 0.67$ & $17 \pm 0.29$ & $36 \pm 0.15$ & $19 \pm 0.8$ & $37 \pm 0.32$ \\
\hline \multirow{2}{*}{$\begin{array}{l}\text { Predicted } \\
\text { landslides in } \\
\text { testing }\end{array}$} & Yes & $30 \pm 0.63$ & $19 \pm 0.78$ & $27 \pm 0.51$ & $12 \pm 0.41$ & $23 \pm 0.24$ & $12 \pm 0.41$ \\
\hline & No & $20 \pm 0.63$ & $31 \pm 0.78$ & $22 \pm 0.51$ & $38 \pm 0.41$ & $27 \pm 0.24$ & $38 \pm 0.41$ \\
\hline
\end{tabular}

\subsubsection{Landslide susceptibility maps}

The conventional landslide susceptibility map and its histogram of susceptibility levels differs substantially from the conventional plus path dependent and path dependent landslide susceptibility maps and histograms (Figure 5.6). This is in accordance with considerable differences in the performance of conventional susceptibility model with the other two models in terms of AUC and AIC values (Table 5.1). The conventional plus path dependent and path dependent landslide susceptibility maps and histograms show more similar patterns despite the differences in the number of variables implemented in the two models.

Both conventional plus path dependent and path dependent landslide susceptibility maps have more pixels in susceptibility level of 0.2-0.4 (507928 and 325184 pixels on average over 16 time slices, respectively) than conventional landslide susceptibility map (151635 pixels). The pattern is inverse in the susceptibility level of 0.4-0.6 where conventional susceptibility map has more pixels (382289 pixels) in compare with conventional plus path dependent and path dependent landslide susceptibility maps (190671 and 159725 pixels, respectively). The other susceptibility levels (0.-02, 0.6-0.8 and 0.8-1) among the three landslide susceptibility maps are not substantially different. The two landslide susceptibility models that take landslide path dependency into account predict that more places are relatively safe - apparently correctly so (Table 5.2). 

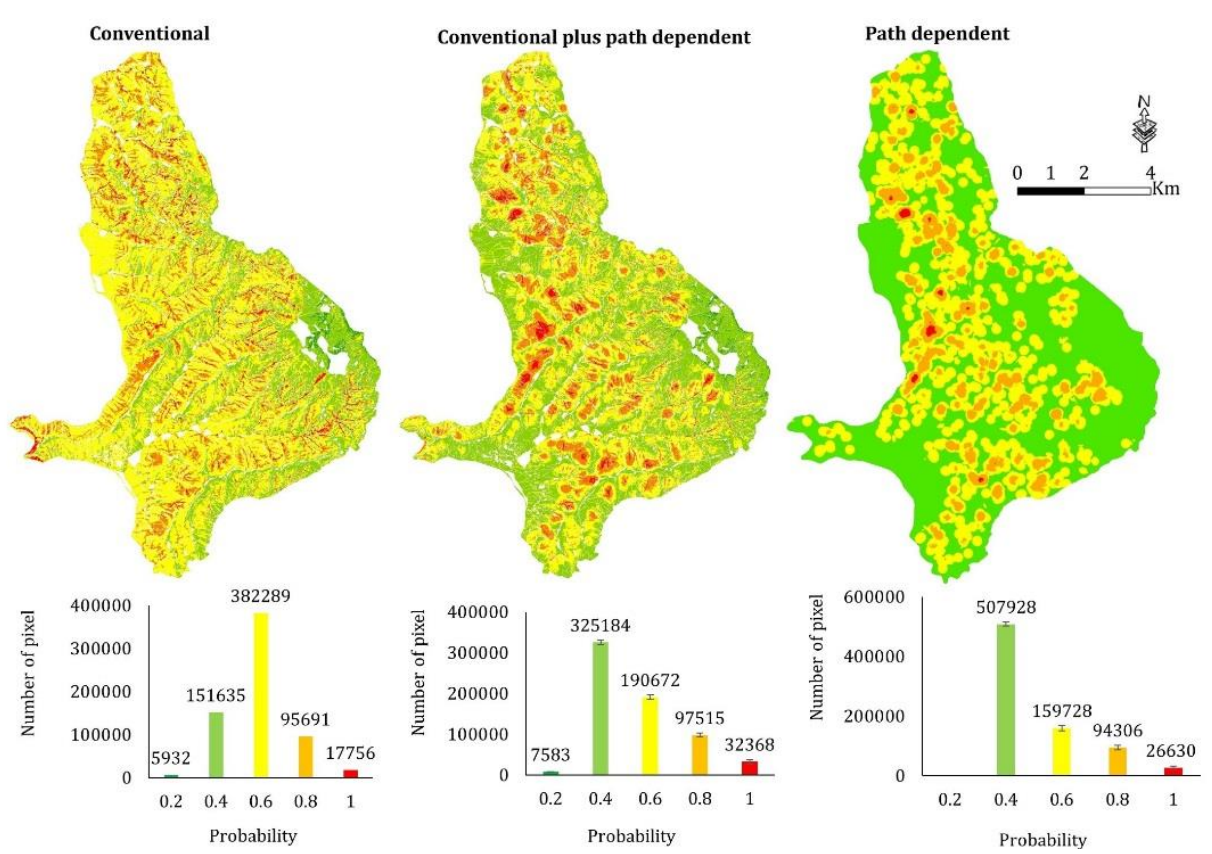

Figure 5.6. Conventional landslide susceptibility map in the left, the conventional plus path dependent landslide susceptibility map (averaged out over 16 time slices) in the middle and path dependent landslide susceptibility map (averaged out over 16 time slices) in the right. The error bars in conventional plus path dependent and path dependent histograms represent standard errors.

The conventional plus path dependent landslide susceptibility maps change over time (Figure 5.7). The changes reflect the decreasing influence of individual earlier landslides, and the sudden increase of influence due to new, more recent landslides. These changes are seen spatially and temporary in the susceptibility maps. 

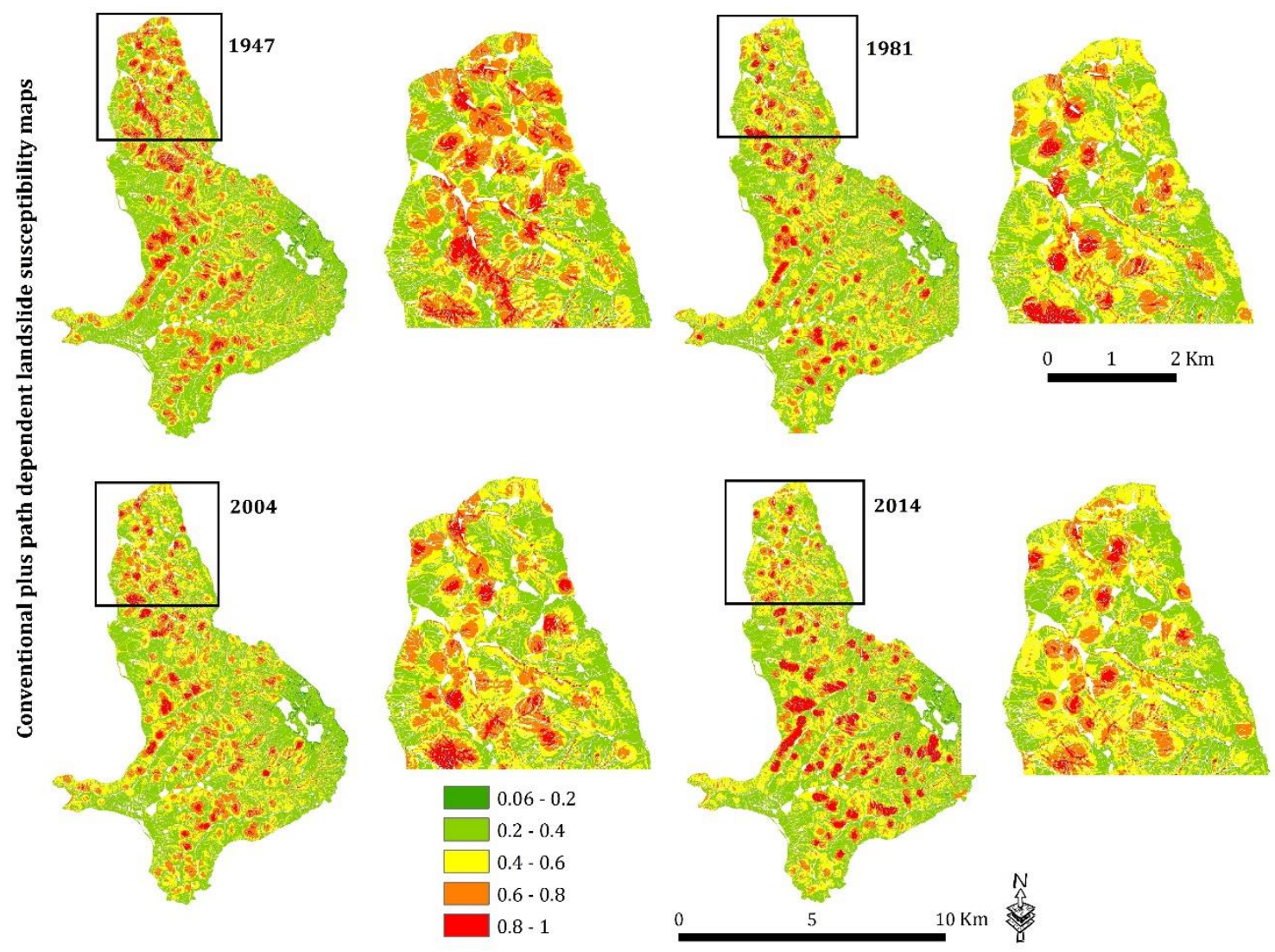

Figure 5.7. Examples of four conventional plus path dependent landslide susceptibility maps in the years of 1947, 1981, 2004 and 2014. Zoomed maps show places where there are large changes in susceptibility over time.

The path dependent landslide susceptibility maps constructed only with the two landslide path dependency variables also change over time (Figure 5.8). The landslide susceptibility maps show only the pure influence of earlier landslides on susceptibility to future landslides. The susceptibility of earlier landslides to future landslides decreases where distance from earlier landslides in space and time increases. 

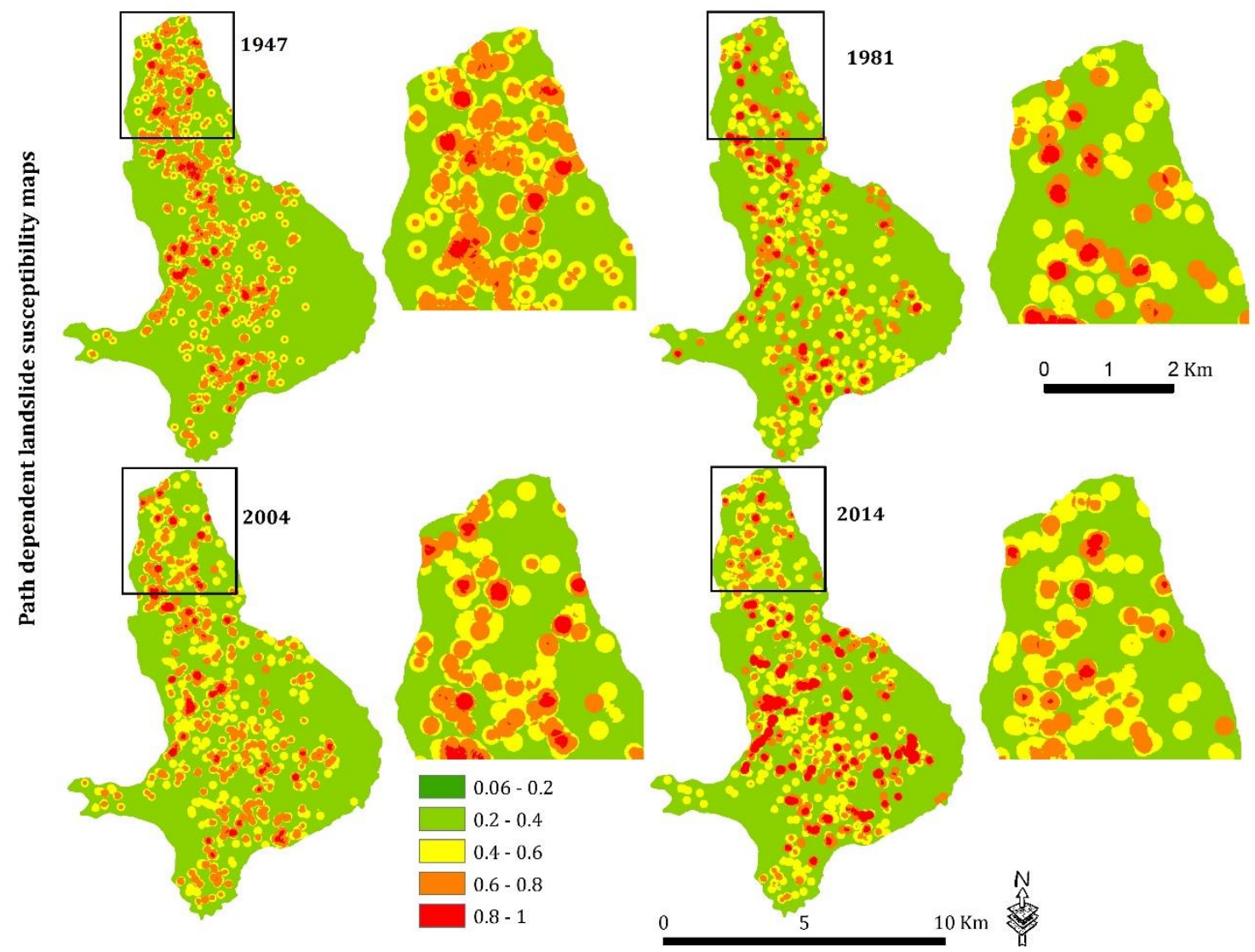

Figure 5.8. Examples of four path dependent landslide susceptibility maps in the years of 1947, 1981, 2004 and 2014. Zoomed maps show the places where there are large changes in susceptibility over time.

\subsection{Discussion}

We first focus on the quantification of landslide path dependency and then discuss its implementation in time-variant path dependent landslide susceptibility modelling. We further discuss the performance of three landslide susceptibility models. At the end, the exportability of landslide path dependency and also the implication of time-variant path dependent landslide susceptibility in landslide hazard and risk assessment will be discussed.

\subsubsection{Spatial-temporal dynamic of landslide path dependency}

The quantified landslide path dependency using Ripley's space-time K function ((Ripley 1976; Diggle et al. 1995) (Figure 5.5) indicates an exponential decay response in the space-time clustering measures values. The susceptibility is high 
within about two decade and 200 meter distance from an earlier landslide, and beyond these spatial-temporal scales, decays exponentially. This means that landslides tend to cluster in the space-time vicinity of an earlier landslide. This is in accordance with our previously quantified landslide path dependency using followup landslide fraction in which the period of landslide path dependency was about two decades (Samia et al. 2017a). The clustering manifests in the form of spatial association among landslides where follow-up landslides occur immediately after and close to a previous landslide (Samia et al. 2017b). Where if possible to monitor the evolution and map the multi-stages activities of a landslide, the occurrence of follow-up landslides could be found either inside the previous landslide, partially overlap with previous landslide or touch the border of previous landslides (Samia et al. 2017b). In this regard, Samia et al. (2017a) discussed that the size of the initial landslide that hits strongly the hillslope plays an important role in the occurrence of follow-up landslides, and hence formation of clusters of landslides. Besides that, they suggested that changes in hydrology of slope destabilized by a landslide could facilitate the occurrence of follow-up and hence clusters of landslides.

The space-time clustering measure also indicates that the susceptibility is dynamic, decays exponentially over time and drops to the background susceptibility level. Using such a dynamic behaviour in landslide susceptibility, we introduced the concept of time-variant landslide susceptibility taking the dynamics of landslide path dependency into account (Samia et al. 2017b; Samia et al. 2017a).

In the computation of space-time clustering, $\mathrm{x}$ and $\mathrm{y}$ were used as geographical coordinates. It could be interesting to define $\mathrm{x}$ in the downslope direction and $\mathrm{y}$ in the slope parallel direction.

\subsubsection{Effect of landslide path dependency on performance of landslide susceptibility models}

We found that taking into account landslide path dependency in a pixel-based conventional landslide susceptibility model (only DEM-derivative variables) improves the performance of landslide susceptibility model substantially. This is reflected in higher AUC and lower AIC values for the conventional plus path dependent landslide susceptibility model (Table 5.1). This confirms our main hypothesis that adding landslide path dependency improves the performance of landslide susceptibility model. This is in accordance with our previous reasoning regarding stronger effect of landslide path dependency in a pixel-based landslide susceptibility model than a slope unit-based landslide susceptibility model (Samia et al. 2018). Landslide path dependency is a local effect in which an earlier landslide could increase the local likelihood of follow-up landslide occurrence. Such a local effect is apparently more visible at pixel resolution and not at slope unit resolution. The strong effect of landslide path dependency on the performance of conventional landslide susceptibility model, is also indicated by the significance of the two landslide path dependency variables $(\mathrm{p}<0.05)$. 
More importantly, the purely path dependent landslide susceptibility model made by the two landslide path dependency variables performs better than the conventional landslide susceptibility model with DEM-derivative variables (Table 5.1). This is potentially interesting in two aspects: on the one hand, using purely DEM-derivatives may not lead to landslide susceptibility model with acceptable results (Table 5.1). On the other hand, the landslide inventory itself can be used to map susceptibility to landsliding without using conditioning attributes (DEMderivatives, geology and land use) that have been conventionally used in landslide susceptibility modelling (Varnes 1984; Guzzetti et al. 2005). Therefore, in datascarce landslide prone areas, only with temporal mapping of landslides (either with point or polygon format) covering a period about two decades close by landslides activities, the path dependent landslide susceptibility can be modelled with reliable results (Table 5.1).

\subsubsection{Temporal validity of susceptibility levels in landslide susceptibility maps}

Landslide susceptibility maps are usually classified into five levels of susceptibility to landslide occurrence. In conventional landslide susceptibility map (Figure 5.6 right), the intensity of different susceptibility levels remains constant over time since the DEM-derivatives used in the model are mono-temporal. Therefore, the temporal validity of susceptibility levels is not known.

In conventional plus path dependent and purely path dependent landslide susceptibility maps (Figure 5.7-5.8), the intensity of different susceptibility levels is time-variant, and changes over time representing the observed exponential decay in susceptibility. The changes are in the places where landslides have already occurred mainly in susceptibility levels ranging from 0.6 to 1 . This suggests that the part of area located in the high susceptibility levels could switch to the low susceptibility levels ( 0 to 0.6 ) after two decades. From practical point of view, the path dependent landslide susceptibility maps should be updated after every landslide event, and in such a cases these maps would be very valid in land use planning.

\subsubsection{Exportability of space-time dynamic of landslide path dependency}

In landslide prone areas where landslides are continuously monitored and mapped in the form of polygon-based multi-temporal inventory, the landslide path dependency can be quantified based on geographical overlap among landslides, and hence used in landslide susceptibility modelling (Samia et al. 2017a; Samia et al. 2018). However, polygon-based multi-temporal landslide inventories are rare to our best of knowledge, and hence geographical overlap among landslides cannot often be computed. In this paper, we proposed using space-time Ripley's K Function to compute landslide path dependency in areas where point-based multi-temporal 
landslide inventories are available. Using such inventories, our space-time clustering measure (eq 6) can be used to quantify path dependency among landslides. In our study area, landslides turned out to cluster within 20 years in the vicinity of an earlier landslide with an exponential decay beyond these spatiotemporal scales (Figure 5.5).

In landslide prone areas with the similar landscape to our study area, the parameters of our space-time clustering measure (eq 7), the two landslide path dependency variables and the actual susceptibility model can be used in landslide susceptibility modelling. In other different landslide prone areas, the theory of landslide path dependency should be explored, and in case of existence of landslide path dependency, landslide path dependency should be quantified and implemented in landslide susceptibility modelling. This has be further studied in areas with point or polygon based multi-temporal landslide inventories (e.g., (Schlögel et al. 2011)).

\subsubsection{Implications of path dependent landslide susceptibility modelling in landslide hazard and risk assessment}

We have already modified the definition of conventional landslide susceptibility modelling (Varnes 1984; Guzzetti et al. 2005) using spatial temporal dynamics of landslide path dependency (Samia et al. 2017b; Samia et al. 2017a) as following:

Landslide susceptibility $_{s, t}=$

$f$ (conditioning attributes $s_{s}$, landslide path dependency $y_{s, t}$ )

Both conventional plus path dependent and path dependent landslide susceptibility models turned out to perform better than conventional landslide susceptibility model (Table 5.1). In both models, availability of a temporal component - reflecting the exponential decay of landslide path dependency - indicates that landslide susceptibility is a dynamic concept. This could challenge further the way that landslide hazard and risk assessments were perceived.

In landslide hazard assessment, landslide susceptibility as a proxy of 'where landslides occur' is combined with the temporal probability of triggers (mainly rainfall) to determine 'when landslides occur' (Guzzetti et al. 2006a). Our proposed path dependent landslide susceptibility (eq 8) suggests that the space-time dynamics of landslide path dependency need to be considered in combination with temporal information of landslide triggers in the assessment of landslide hazard. According to the space-time dynamics of landslide path dependency (Figure 5.5), immediately after a landslide susceptibility is raised (in the probability levels from 0.6-1), and if intense rainfall occurs, the likelihood of further follow-up landslides could increase further . After a decadal time that an earlier landslide has occurred, the landslide deposit will be possibly more stable, and hence the rainfall may have less influence on an earlier landslide to experience a new follow-up landslide. We believe that the same exponential decay response could be observed when path 
dependent landslide susceptibility is used as the input of landslide hazard assessment.

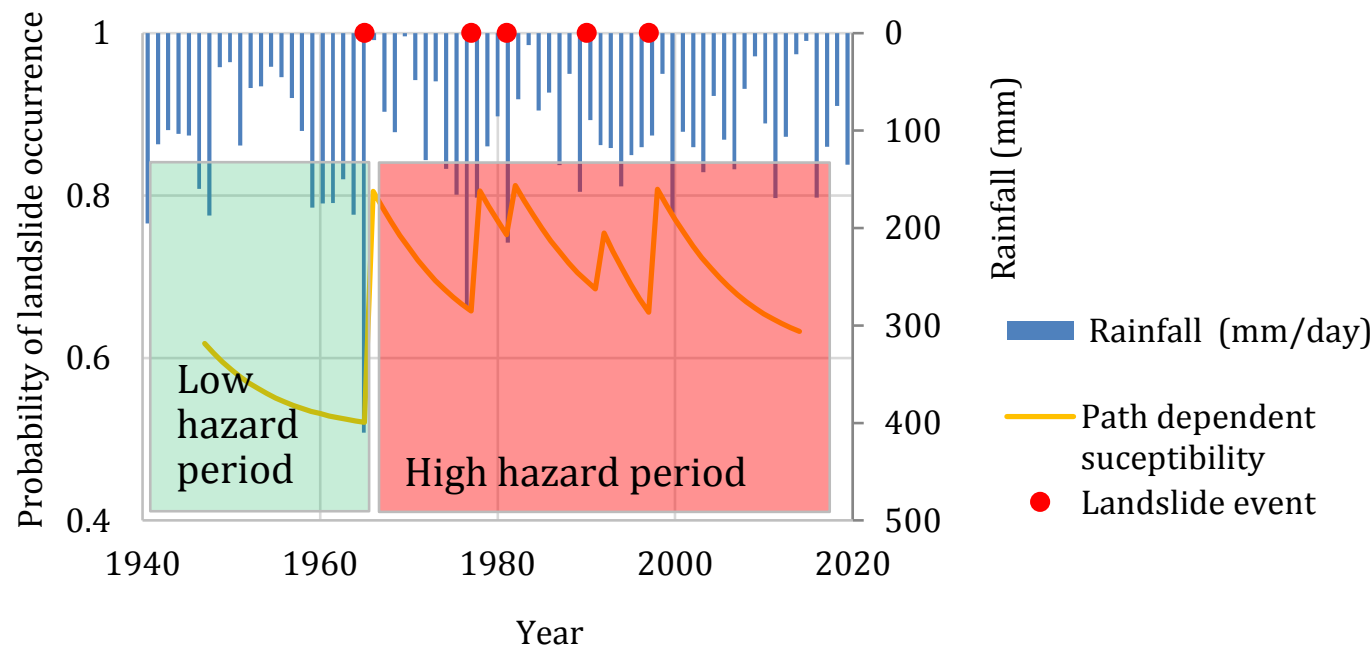

Figure 5.9. Example of the implication of path dependent landslide susceptibility modelling in landslide hazard. When susceptibility is low, the hazard is also low and large rainfall events are needed to trigger new landslides. Then, when susceptibility is high, the hazard is also high and small rainfall events are needed to trigger new landslides.

The effect of our proposed path dependent landslide susceptibility on landslide hazard, may also influence on landslide risk assessment. Landslide susceptibility as a component of landslide hazard, is also the initial step towards landslide risk assessment (Varnes 1984; Guzzetti 2000; Crozier and Glade 2005; Van Westen et al. 2006):

\section{Landslide risk $=$}

landslide hazard $\times$ vulnerability $\times$ value of exposed elements at risk

According to space-time dynamics of landslide path dependency (Figure 5.5), the susceptibility after occurrence of a landslide decreases over time. In this context, vulnerability of elements at risk (e.g., buildings and roads) at the time of landslide occurrence is high, could stay high within about 10 years (due to the possibility of occurring follow-up landslides in the vicinity of initial landslide (Samia et al. 2017b; Samia et al. 2017a)), and after that may decrease. This has to be further studied in a landslide risk assessment using path dependent landslide susceptibility in combination with landslide triggers information. Such an landslide risk assessment could help decision makers and land use planners in short-term planning of regions susceptible to landsliding. 


\subsection{Conclusions}

We quantified landslide path dependency using space-time Ripley's K Function in a point-based multi-temporal landslide inventory in Collazzone study area in Italy. We computed then the measure of space-time clustering reflecting spatial and temporal distances of every pixel from a landslide. We found that space-time clustering measure follows an exponential decay response indicating high degree of clustering among landslides within a temporal scale of 10 years and a spatial distance of $200 \mathrm{~m}$. We added such an exponential decay effects of landslide path dependency into landslide susceptibility modelling. We constructed three landslide susceptibility models: a conventional landslide susceptibility with DEMderivatives, a conventional plus path dependent susceptibility with DEMderivatives and landslide path dependency variables, and a purely path dependent landslide susceptibility with only landslide path dependency variables. We found that considering landslide path dependency improves the performance of landslide susceptibility model. Both conventional plus path dependent and path dependent landslides susceptibility models perform better than conventional landslide susceptibility model. Taking into accounting landslide path dependency effects in landslide susceptibility make the landslide susceptibility model time-variant where susceptibility changes over time. We stress that landslide susceptibility modelling should take the effect of landslide path dependency into account since it provides an estimation of temporal validation of different probability levels in landslide susceptibility map. We proposed that taking into account the time-variant path dependent landslide susceptibility may improve landslide hazard and risk assessment.

Acknowledgements: This research is part of J Samia PhD project at Wageningen University and Research, funded by Ministry of Science, Research and Technology of Iran, Laboratory of Geo-Information Science and Remote Sensing and Soil Geography and Landscape group of Wageningen University supported by Geography Department of Kansas State University. 



\title{
Chapter 6
}

\author{
Synthesis
}




\subsection{Main results}

The overall objective of this $\mathrm{PhD}$ thesis is to assess the effect of landslides on susceptibility of future landslides. To achieve this objective, four research questions have been formulated and discussed in chapters 2 - 5 respectively. First, the main results of each research question will be summarized, after which a reflection on the results and implications is presented. Next, recommendations for further research are proposed.

\subsubsection{What are the indications of landslides following previous landslides?}

Monitoring, documenting and multi-temporal mapping of landslides support exploring multi-stage evolutionary activities of landslides. Using a detailed multitemporal landslide inventory for the Collazzone area (Italy), I used spatial overlap as an indication for landslides following previous landslides (Chapter 2). In our study area, there is a considerable amount of overlap among landslides. In fact, the values of the overlap index (Chapter 2)- reflecting the spatial overlap between landslides over time - is high for time slices within a decade of each other. This indicates that the history of landslides positively affects the future susceptibility. Such a landslide path dependency effect leads to the occurrence of follow-up landslides that are spatially associated with previous landslides. In addition, followup landslides have different size, shape and frequency-area statistics than nonfollow-up landslides.

These results show that there is indeed path dependency among landslides reflecting the multi-stage activities of landslides. This means that landslides leave legacies in the environment which lead to an increased probability of occurrence of follow-up landslides. Although external triggers are important in the occurrence of landslides, the legacy of landslides could enhance chances of follow-up landslides in combination with external triggers. The short-term positive effect of landslide path dependency on the future susceptibility indicates that intensity of susceptibility is dynamic and changes over time. This suggests that landslide susceptibility can no longer be seen as a purely spatial concept (Guzzetti et al. 2005) but needs to consider the dynamic effect of landslide path dependency into account.

\subsubsection{What are the characteristic spatial temporal scales in landslide path dependency?}

In Chapter 2, spatial overlap was found as an indication of landslide path dependency. This was further used to quantify the strength and duration of landslide path dependency in polygon-based (in Chapter 3), and point-based (in Chapter 5) multi-temporal landslide inventories.

In Chapter 3, follow-up landslide fraction - reflecting relative number of follow-up landslides over time - was used to quantify the effect of landslide path dependency. The results indicated that the effect of landslide path dependency is strong after an 
initial landslide over a period of 10 years and decays exponentially over time. Susceptibility was found to increase by a factor of about 15 immediately after a landslides, reflecting the positive and strong effect of a previous landslide on susceptibility. This strength tended to decrease to $25 \%$ of the background susceptibility after 10 years, and was practically absent after 40 years.

In Chapter 5, space-time Ripley's K Function - reflecting the degree of clustering was used to quantify the strength and duration of space-time clustering between landslides. The results confirmed the findings mentioned above, and indicated that landslides tend to cluster in a decadal period and in 200 meter vicinity of a previous landslides.

The findings related to the second research question confirm that landslide susceptibility is not a pure spatial concept (Guzzetti et al. 2005). Instead it is timevariant and should take landslide path dependency effect into account. Furthermore, a decadal positive effect of landslide path dependency on future susceptibility in the vicinity of a previous landslide, proposes a decadal time frame for temporal validity of susceptible levels in landslide susceptibility maps. For about a decade after its occurrence, a large previous landslide could destabilize a hillslope, trigger the occurrence of follow-up landslides, and hence form clusters of landslides that may otherwise not have happened.

\subsubsection{Can we predict the occurrence of future landslides as a function of properties of previous landslides?}

In chapter 3, the differences between two geometric (size and shape) and nine topographical properties (DEM-derivatives) of landslides experiencing follow-up landslides and landslides not experiencing follow-up landslides were compared using one-way analysis of variance. In our study area, larger and rounder landslides in wet places appeared more likely than others to experience follow-up landslides. Among topographical properties, the values of Topographic Wetness Index (TWI), vertical distance to the channel network, the relative slope position and absolute profile curvature differed statistically between landslides with and without followup landslides. The higher value of TWI indicated the positive effect of humidity in the occurrence of follow-up landslides. Such an effect was also confirmed in a field exploration of landslides in the study area with the formation of small ponds on and in the landslide deposits.

Area Under Curve (AUC) values reflect model accuracy and rage between 0.5 ( random performance) to 1 (perfect performance). A combination of geometric and topographic properties, was found to predict the occurrence of follow-up landslides with AUC value of 0.64 . This was slightly better than using merely geometric properties or topographic properties with AUC values of 0.60 and 0.57, respectively.

In summary, combination of geometric and topographic properties of landslides could be used to predict the occurrence of follow-up landslides with acceptable 
model performance. The results also suggest that only the geometric properties of landslides could be used for prediction of follow-up landslides leading to similar model performance when using topographic properties. This emphasizes the needs for precise mapping and documenting of landslides in order to have accurate information regarding the geometrical properties of landslides. However, and with the respect to the low values of AUC, the prediction of occurrence of follow-up landslides remains a challenging task.

\subsubsection{Does including landslide path dependency improve landslide susceptibility modelling?}

\section{At slope unit scale: no substantial improvement}

In chapter 4, two landslides path dependency variables were computed: spatial probability that an earlier landslide will cause a follow-up landslide and susceptibility temporal decay.

Adding these variables into a large set of conditioning attributes conventionally being used in landslide susceptibility modelling, did not improve model performance considerably. This was reflected in the similar model performance between conventional and conventional plus path dependent landslide susceptibility models (AUC of 0.775 and 0.773 respectively), and similar patterns in their associated landslide susceptibility maps.

However, the use of only the two landslide path dependency variables in landslide susceptibility modelling indicated an acceptable model performance (AUC $=0.688$ ). Besides that, the resulted landslide susceptibility map was found to be substantially different from conventional and conventional plus path dependent landslide susceptibility maps.

All in all, these findings indicate that landslide path dependency is a local effect in which previous landslides positively effects on the future susceptibility. Considering such a local effect may not be effective to improve the performance of landslide susceptibility model with coarse mapping units such as slope unit. Apparently, in landslide susceptibility model using coarse mapping units, the conditioning attributes take most of the variations by the model, and hence the predictive value of landslide path dependency could be trivial.

Moreover, landslide susceptibility modelling can apparently be performed using only landslide path dependency. This can be considered as a simple, time and cost efficient landslide susceptibility modelling approach in the scarce-data landslide prone areas. With this, the needs of using large sets of environmental information that are traditionally used in landslide susceptibility modelling (Guzzetti et al. 2005), can be avoided. Such environmental information may not be available or difficult to obtain in landslide prone areas. Our path dependent landslide susceptibility modelling suggests that landslide path dependency can simply be derived from multi-temporal landslide inventory, and used in landslide 
susceptibility modelling leading to acceptable model performance and results. However, in case of availability of environmental information, adding landslide path dependency could lead to very reliable landslide susceptibility modelling and map.

\section{At pixel scale: substantial improvement}

In Chapter 5, two landslide path dependency variables from point-based multitemporal landslide inventory were computed reflecting the degree of space-time clustering measure for every pixel.

Adding these variables along with DEM-derivatives into landslide susceptibility model improved the performance of landslide model substantially (AUC $=0.749$ ). It was also found that a purely landslide susceptibility model constructed with two landslide path dependency variables performed better $(\mathrm{AUC}=0.721)$ than a landslide susceptibility model constructed by DEM-derivatives (AUC $=0.672$ ). In addition, landslide susceptibility maps taking the effect of landslide path dependency into account indicated similar patterns in the susceptibility levels. However, landslide susceptibility maps with landslide path dependency effect were substantially different than landslide susceptibility map without landslide path dependency effect.

These findings indicate that pixel-based landslide susceptibility models could be improved significantly by implementing landslide path dependency effect. In such fine resolution mapping units, the local effects of path dependency are better captured than DEM-derivatives by the model, and hence improve the performance of susceptibility models. The results also suggest that combination of landslide path dependency with DEM-derivatives leads to high model performance in pixel-based landslide susceptibility models. This reduces the need for difficult to obtain and update conditioning attributes such as geology and land use traditionally being used in landslide susceptibility modelling.

Considering the importance of landslide path dependency in improving performance of landslide susceptibility models, I stress the need for regular multitemporal monitoring and mapping of landslides. Also, the type of presenting landslides in the landslide inventories could be point or polygon but the time of mapping or preferably occurrence of landslides should be specified. 


\subsection{Reflection}

The main scientific contributions to the field of landslide susceptibility modelling include quantifying landslide path dependency (Chapter 2, 3 and 5) and modification of the purely spatial concept of landslide susceptibility modelling with introducing time-variant path dependent landslide modelling (Chapter 2, 3, and 5). For the first time, the path dependent response among landslides - reflecting the evolutionary activities of landslides - was quantified using polygon- and pointbased multi-temporal landslide inventories (Chapter 2, 3, and 5). Using this information, we demonstrated the intensity of susceptibility is not time-invariant but changes over a decadal time scale. Besides that, implementing the effect of landslide path dependency in landslide susceptibility modelling not only improved the performance of the model but made landslide susceptibility model and map dynamic and time-variant (Chapter 4 and 5). The following sections reflect on key aspects of landslide path dependency and modelling of landslide susceptibility, mapping of landslide inventory, and discuss the further implications and opportunities in landslide studies.

\subsubsection{Role of landslide path dependency in the evolutionary process of landsliding}

This thesis introduced a new time-related landslide path dependency effect as a factor contributing to the susceptibility of follow-up landslides. The inspiration and motivation of this work originates from the 'path dependency' concept within complexity theory (Phillips 2006). Landslides occur in susceptible hydro-sociogeomorphological systems with complex interactions among external triggers (e.g., rainfall and earthquake), human interventions and the conditioning attributes of hillslopes. In such a system with complex interactions among different triggers, prediction of landslide occurrence is a difficult task. In this thesis, I found that there is path dependency among landslides, and that means that the history of landslides partly determines the future susceptibility to landslide occurrence. Such a path dependency view was already used as a key assumption in landslide susceptibility modelling: 'past and present are the keys for the future' (Varnes 1984; Carrara et al. 1991). However, this assumption did not consider the effects of landslides themselves on the susceptibility of future landslides. In this context, landslide path dependency indicates that landslides increase the susceptibility to future landslides within a short period of time through their legacies in the environment - whatever these may be. The dynamics of landslide path dependency show that susceptibility of a landslide to a new follow-up landslide is high in a decade with exponential decay response over time (Chapter 2, 3 and 5). In the decadal strong effect of landslide path dependency, the cause of follow-up landslides may not be totally external (rainfall) but previous landslides could facilitate the occurrence of followup landslides, and hence form clusters of landslides through weakening materials and changing slope hydrology (Chapter 3).

This kind of landslide path dependency was not mentioned before but there are findings indicating that old large deep-seated landslide and earthflow keep on 
reactivating over several decades (Parise 2003; Van Den Eeckhaut et al. 2009; Booth et al. 2018). We speculate that the mechanisms behind long-term activities of such landslides might be related to the mechanisms underlying the decadal positive strong effect of landslide path dependency on follow-up landslides occurrence despite the differences in the type, duration and size between these landslides. Land use changes and human interventions in the body of old-deep seated landslides, changes in surface water and its infiltration through high permeability zones in landslides deposits, and internal mass distribution within the deposits of landslides have been found to be the mechanisms for such long-term activates of landslides. Moreover, the exponential decay in the positive effect of landslide path dependency on occurrence of follow-up landslides could be attributed to recovery of vegetation growth and ground strength in landslides affected areas (Lin et al. 2007; Marc et al. 2015). Therefore, such path dependent view and attention to the legacy of previous landslides, in combination with external triggers contribute to better understanding of multi-stage activities in landslides, and hence the evolution of hillslopes.

\subsubsection{Dynamic path dependent landslide susceptibility modelling}

Traditionally, landslide susceptibility has been a spatial concept reflecting 'where landslides occur', assessed as a function of distribution of landslides and conditioning attributes (Guzzetti et al. 1999; Guzzetti et al. 2005). This thesis has shown that landslide susceptibility beside the traditional spatial components, has a time-related landslide path dependency component (Chapter 2). Such a component was found to be a positive factor in enhancing susceptibility over a period of a decade with exponential decay response over time (Chapter 3 and 5).

In our study area, consideration of landslide path dependency component improved the performance of landslide susceptibility model and provided dynamic time-variant path landslide susceptibility model (Chapter 4 and 5). The effect of landslide path dependency was substantially strong on the performance of pixelbased landslide susceptibility modelling (Chapter 5) in comparison to trivial impact on the performance of slope unit-based landslide susceptibility modelling (Chapter 4). Given that landslide path dependency is the local effect of landslides on landslides, consideration of such an effect can be stronger to improve the performance of pixel-based rather than slope unit-based landslide susceptibility modelling.

Besides that, the exponential decay response in landslide path dependency adds an important consideration on temporal validity of susceptibility levels presented in landslide susceptibility maps. The susceptibility levels in landslide susceptibility maps have always been considered to be valid for an indefinite period of time. This thesis has shown that (Chapter 3 and 5) that intensity of susceptibility is dynamic reflecting the exponential decay response in landslide path dependency. Considering such a dynamic temporal effect in landslide susceptibility modelling, could shift susceptibility levels when landslide susceptibility maps are updated over time. This needs monitoring and multi-temporal mapping of landslides in order to reflect landslide path dependency effect in landslide susceptibility maps. 
This consideration is simpler in comparison to the time and cost demanding updating of dynamic landslide causal factors (e.g., lands use) and updating landslide inventory maps (Meusburger and Alewell 2009).

Another positive aspect of landslide path dependency is the ability to model landslide susceptibility properly without the need of environmental data which is traditionally being used in landslide susceptibility modelling. In our study area, a pixel-based landslide susceptibility model constructed only with landslide path dependency performed better than pixel-based landslide susceptibility model constructed with DEM-derivatives (Chapter 5). This adds an important consideration into the simplicity of landslide susceptibility modelling by only using landslide path dependency derived from landslide inventories. This can be considered in the preparation of landslide susceptibility maps in data-scarce landslide prone areas where conditioning attributes (e.g., land use and geology) and DEM-derivatives are not available or difficult to obtain. However, in the case of availability of these data, and combination with landslide path dependency, this could lead to more reliable landslide susceptibility models and maps.

In landslide prone areas similar to Collazzone study area, the parameters of our landslide path dependency (Chapter 3 and 5) can directly be used to compute landslide path dependency variables to be implemented in landslide susceptibility modelling. In other landslide prone areas, landslide path dependency can be characterized and quantified with our two developed measures, follow-up landslide fraction (Chapter 3 ) and space-time clustering measure (Chapter 5).

\subsection{Implications of landslide path dependency}

In this section, I will discuss the possible wider implications of landslide path dependency, with respect to multi-temporal mapping, hazard and risk assessment and regional planning.

\subsubsection{Multi-temporal monitoring and mapping of landslides}

I stress the importance of accurate monitoring and multi-temporal mapping of landslides in order to explore the effect of landslide path dependency on future landslides (Chapter 2 and 3). In mapping of landslides, the attention has been given more to the spatial distribution of landslides presented in several landslide inventories worldwide. However, only a few - to the best of our knowledge landslide inventories have given the attention to the time of landslide occurrence or mapping (Guzzetti et al. 2006; Schlögel et al. 2011).

In my thesis, I demonstrated that landslides affect future landslide susceptibility for about a decade (Chapter 3 and 5). To assess such an effect, polygon-based or pointbased multi-temporal landslide inventories are essential. In polygon-based multitemporal landslide inventories, landslide path dependency could be computed using our proposed follow-up landslide fraction $(\rho)$ presented in Chapter 3. In point-based multi-temporal landslide inventories, our proposed space-time 
clustering measure (Chapter 5) derived from space-time Ripley's K Function, could be used to computed landslide path dependency effect. Such inventories should contain time of mapping or occurrence of landslides over a period between 10 to 20 years given a decadal positive effect of landslide path dependency.

Multi-temporal landslide inventories are rare; beside the unique Collazzone dataset, I am aware of only 1 other in Maily-Say valley in Kyrgyzstan (Schlögel et al. 2011). I expect that, with increasing availability of high resolution remote sensing imagery, more multi-temporal landslide inventories will be prepared in the coming decade.

\subsubsection{Impact of landslide path dependency on landslide hazard and risk assessment}

Landslide susceptibility is the key element towards the assessment of landslide hazard and risk (Martha et al. 2013). The new dynamic path dependent landslide susceptibility which has been found in Chapter 2, 3 and 5, could influence on the ways that landslide hazard have been assessed so far. In landslide hazard, a combination of landslide susceptibility with the temporal information of landslide triggers (rainfall) are used to estimate spatial and temporal probabilities of landslide occurrence (Guzzetti et al. 1999). The landslide susceptibility used in landslide hazard, has been so far static without taking the landslide path dependency effect into account. Considering dynamic path dependent landslide susceptibility in combination with temporal information of landslide triggers may lead to different assessment of landslide hazard. As a potential consequence, in the decadal positive effect of landslides on future landslide susceptibility, less rainfall could even further increase the likelihood of follow-up landslide occurrence. Opposite to this, when the effect of landslide path dependency decreases, more intense or prolonged rainfall are needed for future landslides to occur. In other words, recent landsliding may increase susceptibility and thus balance a period of low rainfall, or make a period of high rainfall worse.

The traditional way of landslide risk assessment is a function of landslide hazard, vulnerability and the value of exposed elements at risk (Van Westen et al. 2006). This way of landslide risk assessment could be influenced by time-variant path dependent landslide susceptibility implemented in landslide hazard. Besides that, the vulnerability of elements (e.g., roads and buildings at risk) as a component of landslide risk could be also dynamic by taking time-variant path dependent susceptibility into account.

In the decadal positive effect of previous landslides on future susceptibility where the likelihood of occurrence of follow-up landslides is high, the vulnerability of elements could even further increase. Conversely, when the effect of previous landslides decreases, the vulnerability of elements could drop to the background vulnerability level. Such dynamic behaviours of landslide path dependency and its possible effect on vulnerability needs to be considered in the land risk mitigation strategies. 


\subsubsection{Operational implication of landslide path dependency in land use planning}

Regional development planning contains land and resources allocation at regional levels, project design and implementation like building infrastructures and new residential areas at local community level or site-specific areas (De Graff et al. 2012). Such a planning with global climate change and increasing number of population leading to the intensive use of land and environment, needs to take the destructive effects of landslides into account. In this context, landslide susceptibility maps play a key role for decision makers and awareness of societies living in landslide prone areas. Our landslide susceptibility maps can be simply produced using landslide path dependency and DEM-derivatives in data-scarce areas (Chapter 5). The resulted landslide susceptibility maps can be understandable by societies, useful for decision makers and land use planners to avoid or mitigate the negative impacts of landslides.

Our modified landslide susceptibility modelling (Chapter 2) includes static consideration of conditioning attributes changing over space and the dynamic landslide path dependency effect changing over space and time. These static and dynamic components of landslide susceptibility may complicate land use planning since landslide susceptibility maps need to be updated in areas with ongoing dependencies among landslides. In such areas, and for long-term land use planning, the static component of landslide susceptibility could be more useful for the preparation of landslide susceptibility maps. In this context, landslides susceptibility maps can be provided with the relations between conditioning attributes and landslides distribution. However, the dynamic component of landslide susceptibility needs to be considered in short-term land use planning and investment such as putting irrigation systems. Besides that, taking dynamics of landslide path dependency into account could contribute to the short-term prevention and mitigation strategies to avoid further possible occurrence of followup landslides.

\subsection{Future research}

Based on the findings of this thesis, I provide an outlook on possible future research topics below:

1. The mechanisms behind the dynamics of landslide path dependency should be further studied in order to understand multi-stage activates in landslides. The dynamics of landslide path dependency showed that susceptibility remains high after occurrence of a landslide for about a decade with an exponential decay response over time. Understanding the reason for such a dynamic response in landslide path dependency could contribute to better prediction of future landslide occurrence. In this respect, properties of landslides deposits, depth of sliding planes, changes in water content, bulk density and sediment compaction in deposits of landslides should be investigated in the field, The findings can be used in 
physically-based landslide susceptibility modelling to assess potential further instability of landslides using infinite slope stability model. Besides that, regular multi-temporal monitoring and mapping of landslides and their legacies should also be studied. To this aim, multi-temporal remote sensing imagery, interferometric techniques, LIDAR and Unmanned Aerial Vehicle (UAV) can be used. These tools and techniques provide valuable information to assess the legacy of landslides on hillslopes by monitoring changes in land cover, morphology of hillslopes and comparison of topographic parameters using multi-temporal DEM before and after landslides occurrence.

2. I used logistic regression as a reference statistically-based model to implement the effect of landslide path dependency on landslide susceptibility modelling. Given the variety of quantitative approaches being used in landslide susceptibility modelling, it could be worth to include the effect of landslide path dependency in some other statistical approaches. The findings can be compared with the results of our logistic regression-based path dependent landslide susceptibility modelling.

3. I believe that our path dependent thinking and view on landsliding and its effect on landslide susceptibility is generic. However, the existence and dynamic of landslide path dependency in other landslide prone areas should be investigated and compared with the dynamics of our landslide path dependency. Similar path dependency dynamics in several landslide prone areas would stimulate the global attention of landslide path dependency view and its impact on landslide susceptibility modelling.

4. The optimal method of implementing dynamic path dependent landslide susceptibility as the input in landslide hazard and risk assessment could be the subject of further research. In this context, the results can be compared with the results of landslide hazard and risk assessment where static traditional landslide susceptibility are used as the input. 


\section{References}

Abella EAC, Van Westen CJ (2008) Qualitative landslide susceptibility assessment by multicriteria analysis: a case study from San Antonio del Sur, Guantánamo, Cuba. Geomorphology 94:453-466.

Akaike H (1974) A new look at the statistical model identification. IEEE transactions on automatic control 19:716-723.

Akaike $H$ (1998) Information theory and an extension of the maximum likelihood principle. In: Selected Papers of Hirotugu Akaike. Springer, pp 199-213.

Aleotti P, Chowdhury R (1999) Landslide hazard assessment: summary review and new perspectives. Bulletin of Engineering Geology and the Environment 58:21-44. doi:10.1007/s100640050066.

Alvioli M, Marchesini I, Reichenbach P, Rossi M, Ardizzone F, Fiorucci F, Guzzetti F (2016) Automatic delineation of geomorphological slope units with r.slopeunits v1.0 and their optimization for landslide susceptibility modeling. Geosci Model Dev 9:3975-3991. doi:10.5194/gmd-9-3975-2016.

Ardizzone F, Cardinali M, Carrara A, Guzzetti F, Reichenbach P (2002) Impact of mapping errors on the reliability of landslide hazard maps. Nat Hazards Earth Syst Sci 2:3-14. doi:10.5194/nhess-2-3-2002.

Ardizzone F, Cardinali M, Galli M, Guzzetti F, Reichenbach P (2007) Identification and mapping of recent rainfall-induced landslides using elevation data collected by airborne Lidar. Natural Hazards and Earth System Science 7:637-650

Ardizzone F et al. (2013) Very-High Resolution Stereoscopic Satellite Images for Landslide Mapping. Landslide Science and Practice 1:95-101. doi:doi: 10.1007/978-3-642-31325-7_12.

Ardizzone F et al. (2013) Very-high resolution stereoscopic satellite images for landslide mapping. In: Landslide Science and Practice. Springer, pp 95-101.

Atkinson PM, Massari R (1998) Generalised linear modelling of susceptibility to landsliding in the Central Apennines, Italy Computers \& Geosciences 24:373385. doi:http://dx.doi.org/10.1016/S0098-3004(97)00117-9.

Barredo JI, Benavides A, Hervás J, Van Westen CJ (2000) Comparing heuristic landslide hazard assessment techniques using GIS in the Tirajana basin, Gran Canaria Island, Spain. International Journal of Applied Earth Observation and Geoinformation 2000:9-23. 
Baum R, Reid M Ground water isolation by low-permeability clays in landslide shear zones. In: Landslides in Research, Theory and Practice: Proceedings of the 8th International Symposium on Landslides held in Cardiff on 26-30 June 2000, 2000. Thomas Telford Publishing, pp 1: 139-144.

Beguería S (2006) Validation and evaluation of predictive models in hazard assessment and risk management. Natural Hazards 37:315-329.

Bock M, Köthe R (2008) Predicting the depth of hydromorphic Soil Characteristics influenced by Ground Water. SAGA-Seconds Out 19:13-22.

Booth AM, McCarley J, Hinkle J, Shaw S, Ampuero JP, Lamb MP (2018) Transient reactivation of a deep-seated landslide by undrained loading captured with repeat airborne and terrestrial lidar. Geophysical Research Letters 45:48414850.

Borgomeo E, Hebditch KV, Whittaker AC, Lonergan L (2014) Characterising the spatial distribution, frequency and geomorphic controls on landslide occurrence, Molise, Itlay. Geomorphology 226:148-161.

doi:http://dx.doi.org/10.1016/j.geomorph.2014.08.004.

Brabb EE (1984) Innovative approaches to landslide hazard and risk mapping.

Brabb EE (1984) Innovative approaches to landslide hazard mapping, Proceedings 4th International Symposium on Landslides, Toronto, vol. 1 (1984), pp. 307324.

Brabb EE (1991) The world landslide problem. In: Episodes, vol 14. US International Union of Geological Sciences (IUGS), pp 52-61.

Brenning A (2005) Spatial prediction models for landslide hazards: Review, comparison and evaluation. Natural Hazards and Earth System Science 5:853-862.

Brunsden D (1993) Mass movement; the research frontier and beyond: a geomorphological approach. Geomorphology 7:85-128.

doi:http://dx.doi.org/10.1016/0169-555X(93)90013-R.

Cardinali M, Antonini G, Reichenbach P, Guzzetti F (2001) Photo-geological and landslide inventory map for the Upper Tiber River basin. CNR, Gruppo Nazionale per la Difesa dalle Catastrofi Idrogeologiche, Publication.

Cardinali M, Ardizzone F, Galli M, Guzzetti F, Reichenbach P Landslides triggered by rapid snow melting: the December 1996-January 1997 event in Central Italy. In: Proceedings 1st Plinius Conference on Mediterranean Storms, 2000. Citeseer, pp 439-448.

Cardinali M, Carrara A, Guzzetti F, Reichenbach P (2002) Landslide hazard map for the Upper Tiber River basin. CNR, Gruppo Nazionale per la Difesa dalle Catastrofi Idrogeologiche, Publication.

Cardinali M, Galli M, Guzzetti F, Ardizzone F, Reichenbach P, Bartoccini P (2006) Rainfall induced landslides in December 2004 in south-western Umbria, central Italy: types, extent, damage and risk assessment. Nat Hazards Earth Syst Sci 6:237-260. doi:10.5194/nhess-6-237-2006. 
Carrara A, Cardinali M, Detti R, Guzzetti F, Pasqui V, Reichenbach P (1991) GIS techniques and statistical models in evaluating landslide hazard. Earth surface processes and landforms 16:427-445.

Carrara A, Cardinali M, Guzzetti F (1992): Uncertainty in assessing landslide hazard and risk. ITC Journal 2:172-183.

Carrara A, Cardinali M, Guzzetti F, Reichenbach P (1995) GIS technology in mapping landslide hazard. In: Geographical Information Systems in Assessing Natural Hazards, . Kluwer Academic Publisher, Dordrecht, The Netherlands (1995), pp. 135-175, pp 135-175.

Carrara A, Guzzetti F, Cardinali M, Reichenbach P (1999) Use of GIS Technology in the Prediction and Monitoring of Landslide Hazard. Natural Hazards 20:117-135. doi:10.1023/a:1008097111310.

Carson MA, Kirkby MJ (1972) Hillslope form and process.

Chen C-Y (2009) Sedimentary impacts from landslides in the Tachia River Basin, Taiwan. Geomorphology 105:355-365.

doi:http://dx.doi.org/10.1016/j.geomorph.2008.10.009.

Chiang S (2015) The analysis of size and shape of rainfall-induced landslides. Paper presented at the EGU General Assembly 2015, Vianna, 2015.

Conrad 0 et al. (2015) System for automated geoscientific analyses (SAGA) v. 2.1. 4. Geoscientific Model Development 8:1991-2007.

Cox DR (1958) The regression analysis of binary sequences. Journal of the Royal Statistical Society Series B (Methodological):215-242.

Crozier MJ (1986) Landslides: Causes, Consequences \& Environment. Croom Helm Pub, London.

Crozier MJ, Glade T (2005) Landslide hazard and risk: issues, concepts and approach. Landslide hazard and risk:1-40.

Cruden D (1996) Cruden,D.M., Varnes, D.J., 1996, Landslide Types and Processes, Special Report, Transportation Research Board, National Academy of Sciences, 247:36-75. Special Report - National Research Council, Transportation Research Board.

D.J. V (1984) Landslide hazard zonation: a review of principles and practice. The Unesco Press, Paris.

Dadson SJ et al. (2004) Earthquake-triggered increase in sediment delivery from an active mountain belt. Geology 32:733-736.

De Graff JV, Romesburg HC, Ahmad R, McCalpin JP (2012) Producing landslidesusceptibility maps for regional planning in data-scarce regions. Natural hazards 64:729-749.

Deng X, Li L, Tan Y (2017) Validation of spatial prediction models for landslide susceptibility mapping by considering structural similarity. ISPRS International Journal of Geo-Information 6:103. 
Densmore AL, Hovius N (2000) Topographic fingerprints of bedrock landslides. Geology 28:371-374.

Diggle PJ, Chetwynd AG, Häggkvist R, Morris SE (1995) Second-order analysis of space-time clustering. Statistical methods in medical research 4:124-136.

Fan L, Lehmann P, Or D (2015) Effects of hydromechanical loading history and antecedent soil mechanical damage on shallow landslide triggering. Journal of Geophysical Research: Earth Surface 120:1990-2015.

Fawcett T (2006) An introduction to ROC analysis. Pattern recognition letters 27:861-874.

Fiorucci F, Ardizzone F, Rossi M, Torri D (2015) The Use of Stereoscopic Satellite Images to Map Rills and Ephemeral Gullies. Remote Sens 7: 14151-14178.

Galli M, Ardizzone F, Cardinali M, Guzzetti F, Reichenbach P (2008) Comparing landslide inventory maps. Geomorphology 94:268-289.

doi:http://dx.doi.org/10.1016/j.geomorph.2006.09.023.

Gavin DG, Hu FS, Lertzman K, Corbett P (2006) Weak climatic control of standescale fire history during the late holocene. Ecology 87:1722-1732.

Geertsema M, Highland L, Vaugeouis L (2009) Environmental Impact of Landslides. In: Sassa K, Canuti P (eds) Landslides - Disaster Risk Reduction. Springer Berlin Heidelberg, Berlin, Heidelberg, pp 589-607. doi:10.1007/978-3-54069970-5_31.

Ghosh S, Carranza EJM, van Westen CJ, Jetten VG, Bhattacharya DN (2011) Selecting and weighting spatial predictors for empirical modeling of landslide susceptibility in the Darjeeling Himalayas (India). Geomorphology 131:3556. doi:https://doi.org/10.1016/j.geomorph.2011.04.019.

Godt JW, Baum RL, Savage WZ, Salciarini D, Schulz WH, Harp EL (2008) Transient deterministic shallow landslide modeling: Requirements for susceptibility and hazard assessments in a GIS framework. Engineering Geology 102:214226. doi:http://dx.doi.org/10.1016/j.enggeo.2008.03.019.

Green DM, Swets JA (1966) Signal detection theory and psychophysics.

Günther A, Van Den Eeckhaut M, Malet J-P, Reichenbach P, Hervás J (2014) Climatephysiographically differentiated Pan-European landslide susceptibility assessment using spatial multi-criteria evaluation and transnational landslide information. Geomorphology 224:69-85.

doi:https://doi.org/10.1016/j.geomorph.2014.07.011.

Guzzetti F (2000) Landslide fatalities and the evaluation of landslide risk in Italy. Engineering Geology 58:89-107.

Guzzetti F (2006) Landslide hazard and risk assessment. Universitäts-und Landesbibliothek Bonn. 
Guzzetti F, Ardizzone F, Cardinali M, Galli M, Reichenbach P, Rossi M (2008) Distribution of landslides in the Upper Tiber River basin, central Italy. Geomorphology 96:105-122.

doi:http://dx.doi.org/10.1016/j.geomorph.2007.07.015.

Guzzetti F, Ardizzone F, Cardinali M, Rossi M, Valigi D (2009) Landslide volumes and landslide mobilization rates in Umbria, central Italy. Earth and Planetary Science Letters 279:222-229. doi:10.1016/j.epsl.2009.01.005.

Guzzetti F, Cardinali M, Reichenbach P (1996) The influence of structural setting and lithology on landslide type and pattern. Environmental \& Engineering Geoscience 2:531-555.

Guzzetti F, Cardinali M, Reichenbach P, Carrara A (2000) Comparing landslide maps: A case study in the upper Tiber River Basin, central Italy. Environmental management 25:247-263.

Guzzetti F, Cardinali M, Reichenbach P, Cipolla F, Sebastiani C, Galli M, Salvati P (2004) Landslides triggered by the 23 November 2000 rainfall event in the Imperia Province, Western Liguria, Italy. Engineering Geology 73:229-245.

Guzzetti F, Carrara A, Cardinali M, Reichenbach P (1999) Landslide hazard evaluation: a review of current techniques and their application in a multiscale study Central Italy. Geomorphology 31:181-216.

doi:http://dx.doi.org/10.1016/S0169-555X(99)00078-1.

Guzzetti F, Galli M, Reichenbach P, Ardizzone F, Cardinali M (2006) Landslide hazard assessment in the Collazzone area, Umbria, Central Italy. Nat Hazards Earth Syst Sci 6:115-131. doi:10.5194/nhess-6-115-2006.

Guzzetti F, Malamud BD, Turcotte DL, Reichenbach P (2002) Power-law correlations of landslide areas in central Italy. Earth and Planetary Science Letters 195:169-183. doi:http://dx.doi.org/10.1016/S0012-821X(01)005891.

Guzzetti F, Mondini AC, Cardinali M, Fiorucci F, Santangelo M, Chang K-T (2012) Landslide inventory maps: New tools for an old problem. Earth-Science Reviews 112:42-66. doi:http://dx.doi.org/10.1016/j.earscirev.2012.02.001.

Guzzetti F, Reichenbach P, Ardizzone F, Cardinali M, Galli M (2006) Estimating the quality of landslide susceptibility models. Geomorphology 81:166-184. doi:10.1016/j.geomorph.2006.04.007.

Guzzetti F, Reichenbach P, Cardinali M, Galli M, Ardizzone F (2005) Probabilistic landslide hazard assessment at the basin scale. Geomorphology 72:272-299. doi:10.1016/j.geomorph.2005.06.002.

Harshburger BJ, Humes KS, Walden VP, Blandford TR, Moore BC, Dezzani RJ (2010) Spatial interpolation of snow water equivalency using surface observations and remotely sensed images of snow-covered area. Hydrological processes 24:1285-1295.

Hergarten S (2003) Landslides, sandpiles, and self-organized criticality. 3:505-514. 
Hergarten S, Neugebauer HJ (1998) Self-organized criticality in a landslide model. 25:801-804.

Hinman SE, Blackburn JK, Curtis A (2006) Spatial and temporal structure of typhoid outbreaks in Washington, DC, 1906-1909: evaluating local clustering with the $\mathrm{G} \mathrm{i}^{*}$ statistic. International Journal of Health Geographics 5:13.

Huang AYL, Montgomery DR (2014) Topographic locations and size of earthquakeand typhoon-generated landslides, Tachia River, Taiwan. Earth Surface Processes and Landforms 39:414-418.

Hutchinson JN (1995) Keynote paper: landslide hazard assessment. Int. symp. on landslides. Christchurch 3:1805-1841.

Igwe 0, Fukuoka $H$ (2015) The effect of water-saturation on the stability of problematic slopes at the Iva Valley area, Southeast Nigeria. Arabian Journal of Geosciences 8:3223-3233. doi:10.1007/s12517-014-1398-7.

Jolliffe IT, Stephenson DB (2003) Forecast verification: a practitioner's guide in atmospheric science. Chichester: Wiley.

Kavzoglu T, Sahin EK, Colkesen I (2014) Landslide susceptibility mapping using GIS-based multi-criteria decision analysis, support vector machines, and logistic regression. Landslides 11:425-439. doi:10.1007/s10346-013-03917.

Kawabata D, Bandibas J (2009) Landslide susceptibility mapping using geological data, a DEM from ASTER images and an Artificial Neural Network (ANN). Geomorphology 113:97-109.

doi:http://dx.doi.org/10.1016/j.geomorph.2009.06.006.

Lan HX, Zhou CH, Wang LJ, Zhang HY, Li RH (2004) Landslide hazard spatial analysis and prediction using GIS in the Xiaojiang watershed, Yunnan, China. Engineering Geology 76:109-128. doi:10.1016/j.enggeo.2004.06.009.

Larsen IJ, Montgomery DR (2012) Landslide erosion coupled to tectonics and river incision. Nature Geoscience 5:468-473.

Lee S, Sambath T (2006) Landslide susceptibility mapping in the Damrei Romel area, Cambodia using frequency ratio and logistic regression models. Environmental Geology 50:847-855. doi:10.1007/s00254-006-0256-7.

Levine N (2006) Crime mapping and the Crimestat program. Geographical analysis 38:41-56.

Lin W-T, Chou W-C, Lin C-Y, Huang P-H, Tsai J-S (2007) Study of landslides caused by the 1999 Chi-Chi earthquake, Taiwan, with multitemporal SPOT images. Canadian Journal of Remote Sensing 33:289-302. doi:10.5589/m07-036.

Lin W-T, Lin C-Y, Chou W-C (2006) Assessment of vegetation recovery and soil erosion at landslides caused by a catastrophic earthquake: A case study in Central Taiwan. Ecological Engineering 28:79-89.

doi:http://dx.doi.org/10.1016/j.ecoleng.2006.04.005. 
Malamud BD, Turcotte DL, Guzzetti F, Reichenbach P (2004) Landslide inventories and their statistical properties. Earth Surface Processes and Landforms 29:687-711. doi:10.1002/esp.1064.

Malamud BD, Turcotte DL, Guzzetti F, Reichenbach P (2004) Landslides, earthquakes, and erosion. Earth and Planetary Science Letters 229:45-59. doi:http://dx.doi.org/10.1016/j.epsl.2004.10.018.

Mancini F, Ceppi C, Ritrovato G (2010) GIS and statistical analysis for landslide susceptibility mapping in the Daunia area, Italy. Natural Hazards and Earth System Sciences 10:1851.

Mann HB, Whitney DR (1947) On a Test of Whether one of Two Random Variables is Stochastically Larger than the Other. The Annals of Mathematical Statistics 18:50-60.

Marc O, Hovius N, Meunier P, Uchida T, Hayashi S (2015) Transient changes of landslide rates after earthquakes. Geology. doi:10.1130/g36961.1.

Martha TR, van Westen CJ, Kerle N, Jetten V, Kumar KV (2013) Landslide hazard and risk assessment using semi-automatically created landslide inventories. Geomorphology 184:139-150.

Martinović K, Gavin K, Reale C (2016) Development of a landslide susceptibility assessment for a rail network. Engineering Geology 215:1-9.

doi:http://dx.doi.org/10.1016/j.enggeo.2016.10.011.

Maruca SL, Jacquez GM (2002) Area-based tests for association between spatial patterns. Journal of Geographical Systems 4:69-83.

doi:10.1007/s101090100075.

Mason SJ, Graham NE (2002) Areas beneath the relative operating characteristics (ROC) and relative operating levels (ROL) curves: Statistical significance and interpretation. Quarterly Journal of the Royal Meteorological Society 128:2145-2166.

Menard S (2000) Coefficients of determination for multiple logistic regression analysis. The American Statistician 54:17-24.

Mergili M, Marchesini I, Alvioli M, Metz M, Schneider-Muntau B, Rossi M, Guzzetti F (2014) A strategy for GIS-based 3-D slope stability modelling over large areas. Geoscientific Model Development 7:2969-2982.

Meunier P, Hovius N, Haines JA (2008) Topographic site effects and the location of earthquake induced landslides. Earth and Planetary Science Letters 275:221-232. doi:http://dx.doi.org/10.1016/j.epsl.2008.07.020.

Meusburger K, Alewell C (2008) Impacts of anthropogenic and environmental factors on the occurrence of shallow landslides in an alpine catchment (Urseren Valley, Switzerland). Nat Hazards Earth Syst Sci 8:509-520. doi:10.5194/nhess-8-509-2008.

Meusburger K, Alewell C (2009) On the influence of temporal change on the validity of landslide susceptibility maps. Natural Hazards and Earth System Sciences 9:1495-1507. 
Meusburger K, Alewell C (2009) On the influence of temporal change on the validity of landslide susceptibility maps. Natural Hazards and Earth System Sciences 9:1495-1507.

Moore ID, Burch GJ (1986) Physical basis of the length-slope factor in the Universal Soil Loss Equation. Soil Science Society of America Journal 50:1294-1298.

Moore ID, Grayson RB (1991) Terrain-based catchment partitioning and runoff prediction using vector elevation data. Water Resources Research 27:11771191.

Neuhäuser B, Damm B, Terhorst B (2012) GIS-based assessment of landslide susceptibility on the base of the weights-of-evidence model. Landslides 9:511-528.

Ohlmacher GC (2007) Plan curvature and landslide probability in regions dominated by earth flows and earth slides. Engineering Geology 91:117134. doi:http://dx.doi.org/10.1016/j.enggeo.2007.01.005.

Parise M (2003) Observation of surface features on an active landslide, and implications for understanding its history of movement. Natural Hazards and Earth System Science 3:569-580.

Parker R, Hancox G, Petley D, Massey C, Densmore A, Rosser N (2015) Spatial distributions of earthquake-induced landslides and hillslope preconditioning in the northwest South Island, New Zealand. Earth Surface Dynamics 3:501.

Parker RN et al. (2011) Mass wasting triggered by the 2008 Wenchuan earthquake is greater than orogenic growth. nature geoscience 4:449.

Petley D (2012) Global patterns of loss of life from landslides. Geology 40:927-930.

Petschko H, Bell R, Brenning A, Glade T (2012) Landslide susceptibility modeling with generalized additive models - Fac- ing the heterogeneity of large regions. Landslides and Engineered Slopes, Protecting Society Through Improved Understanding 1:769-777.

Phillips JD (2006) Evolutionary geomorphology: thresholds and nonlinearity in landform response to environmental change. Hydrol Earth Syst Sci 10:731742. doi:10.5194/hess-10-731-2006.

Ran Q-h, Qian Q, Li W, Fu X-d, Yu X, Xu Y-p (2015) Impact of earthquake-inducedlandslides on hydrologic response of a steep mountainous catchment: a case study of the Wenchuan earthquake zone. Journal of Zhejiang University SCIENCE A 16:131-142. doi:10.1631/jzus.A1400039.

Reichenbach P, Rossi M, Malamud BD, Mihir M, Guzzetti F (2018) A review of statistically-based landslide susceptibility models. Earth-Science Reviews 180:60-91. doi:https://doi.org/10.1016/j.earscirev.2018.03.001.

Remondo J, González-Díez A, Díaz de Terán JR, Cendrero A (2003) Landslide susceptibility models utilising spatial data analysis techniques. A case study from the lower Deba Valley, Guipúzcoa (Spain). Natural Hazards 30:267-279. doi:10.1023/B:NHAZ.0000007202.12543.3a. 
Ripley BD (1976) The second-order analysis of stationary point processes. Journal of applied probability 13:255-266.

Ripley BD (1977) Modelling spatial patterns. Journal of the Royal Statistical Society Series B (Methodological):172-212.

Rossi M, Guzzetti F, Reichenbach P, Mondini AC, Peruccacci S (2010) Optimal landslide susceptibility zonation based on multiple forecasts. Geomorphology 114:129-142.

doi:http://dx.doi.org/10.1016/j.geomorph.2009.06.020

Rossi M, Malamud BD (2014) D. 5.3. Prototype SW for determination of landslide statistics from inventory maps. CNR, KCL.

Rossi M, Reichenbach P (2016) LAND-SE: a software for statistically based landslide susceptibility zonation, version 1.0. Geoscientific Model Development 9:3533.

Ruff M, Czurda K (2008) Landslide susceptibility analysis with a heuristic approach in the Eastern Alps (Vorarlberg, Austria). Geomorphology 94:314-324. doi:10.1016/j.geomorph.2006.10.032

Saboya F, da Glória Alves M, Dias Pinto W (2006) Assessment of failure susceptibility of soil slopes using fuzzy logic. Engineering Geology 86:211224. doi:http://dx.doi.org/10.1016/j.enggeo.2006.05.001

Samia J, Temme A, Bregt A, Wallinga J, Guzzetti F, Ardizzone F, Rossi M (2016) Do landslides follow landslides? Insights in path dependency from a multitemporal landslide inventory. Landslides:1-12.

Samia J, Temme A, Bregt A, Wallinga J, Guzzetti F, Ardizzone F, Rossi M (2017) Characterization and quantification of path dependency in landslide susceptibility. Geomorphology 292:16-24.

Samia J, Temme A, Bregt A, Wallinga J, Guzzetti F, Ardizzone F, Rossi M (2017) Do landslides follow landslides? Insights in path dependency from a multitemporal landslide inventory. Landslides 14:547-558. doi:10.1007/s10346016-0739-x

Samia J et al. (2018) Implementing landslide path dependency in landslide susceptibility modelling. Landslides:1-16.

Santangelo M, Marchesini I, Bucci F, Cardinali M, Fiorucci F, Guzzetti F (2015) An approach to reduce mapping errors in the production of landslide inventory maps. Nat Hazards Earth Syst Sci 15:2111-2126.

doi:10.5194/nhess-15-2111-2015

Santangelo M, Marchesini I, Cardinali M, Fiorucci F, Rossi M, Bucci F, Guzzetti F (2015) A method for the assessment of the influence of bedding on landslide abundance and types. Landslides 12:295-309.

doi:10.1007/s10346-014-0485- $x$ 
Schlögel R, Torgoev I, De Marneffe C, Havenith HB (2011) Evidence of a changing size-frequency distribution of landslides in the Kyrgyz Tien Shan, Central Asia. Earth Surface Processes and Landforms 36:1658-1669.

Schuster R, Highland L (2003) Impact of landslides and innovative landslidemitigation measures on the natural environment. Paper presented at the International Conference on Slope Engineering, Hong Kong, China.

Schuster RL, Highland LM Impact of landslides and innovative landslide-mitigation measures on the natural environment. In: International Conference on Slope Engineering, Hong Kong, China, 2003.

Singh KK, Mehrotra A, Pal K (2014) Landslide detection from satellite images using spectral indices and Digital Elevation Model. Disaster Advances 7:25-32.

Singh RG, Botha GA, Richards NP, McCarthy TS (2008) Holocene landslides in KwaZulu-Natal, South Africa. South African Journal of Geology 111:39-52. doi:10.2113/gssajg.111.1.39

Smith T (2016) Notebook on spatial data analysis. Lecture Note.

Stark CP, Hovius N (2001) The characterization of landslide size distributions. Geophysical Research Letters 28:1091-1094. doi:10.1029/2000gl008527

Student (1908) The Probable Error of a Mean. Biometrika 6:1-25. doi:doi:10.1093/biomet/5.3.351

Taylor F, Brabb EE (1986) Map showing landslides in California that have caused fatalities or at least $\$ 1,000,000$ in damages from 1906 to 1984 . US Geological Survey.

Taylor FE, Malamud BD, Witt A (2015) What Shape is a Landslide? Statistical Patterns in Landslide Length to Width Ratio. Paper presented at the EGU General Assembly 2015, vianna.

Temme AJ, Keiler M, Karssenberg D, Lang A (2015) Complexity and non-linearity in earth surface processes-concepts, methods and applications. Earth Surface Processes and Landforms 40:1270-1274.

Temme AJAM, Keiler M, Karssenberg D, Lang A (2015) Complexity and nonlinearity in earth surface processes - concepts, methods and applications. Earth Surface Process and Landforms 40:1270-1274. doi:10.1002/esp.3712

Tien Bui D, Tuan T, Klempe H, Pradhan B, Revhaug I (2015) Spatial prediction models for shallow landslide hazards: a comparative assessment of the efficacy of support vector machines, artificial neural networks, kernel logistic regression, and logistic model tree. Landslides: 1-18.

doi:10.1007/s10346-015-0557-6

Tien Bui D, Tuan TA, Klempe H, Pradhan B, Revhaug I (2016) Spatial prediction models for shallow landslide hazards: a comparative assessment of the efficacy of support vector machines, artificial neural networks, kernel logistic regression, and logistic model tree. Landslides 13:361-378. doi:10.1007/s10346-015-0557-6 
Tonini M, Pedrazzini A, Penna I, Jaboyedoff M (2014) Spatial pattern of landslides in Swiss Rhone Valley. Natural Hazards 73:97-110.

Trigila A, Iadanza C, Esposito C, Scarascia-Mugnozza G (2015) Comparison of Logistic Regression and Random Forests techniques for shallow landslide susceptibility assessment in Giampilieri (NE Sicily, Italy). Geomorphology 249:119-136. doi:http://dx.doi.org/10.1016/j.geomorph.2015.06.001

Turcotte DL, Malamud BD, Guzzetti F, Reichenbach P (2002) Self-organization, the cascade model, and natural hazards. Proceedings of the National Academy of Sciences 99:2530-2537. doi:10.1073/pnas.012582199

Turcotte DL, Malamud BD, Guzzetti F, Reichenbach P (2006) A general landslide distribution applied to a small inventory in Todi, Italy vol 261. doi:10.1144/gsl.sp.2006.261.01.08

Van Den Eeckhaut M, Moeyersons J, Nyssen J, Abraha A, Poesen J, Haile M, Deckers $J(2009)$ Spatial patterns of old, deep-seated landslides: a case-study in the northern Ethiopian highlands. Geomorphology 105:239-252.

Van Den Eeckhaut M, Poesen J, Dewitte 0, Demoulin A, De Bo H, VanmaerckeGottigny M (2007) Reactivation of old landslides: lessons learned from a case-study in the Flemish Ardennes (Belgium). Soil use and management 23:200-211.

Van Den Eeckhaut M, Poesen J, Govers G, Verstraeten G, Demoulin A (2007) Characteristics of the size distribution of recent and historical landslides in a populated hilly region. Earth and Planetary Science Letters 256:588-603. doi:http://dx.doi.org/10.1016/j.epsl.2007.01.040

Van Den Eeckhaut M, Reichenbach P, Guzzetti F, Rossi M, Poesen J (2009) Combined landslide inventory and susceptibility assessment based on different mapping units: an example from the Flemish Ardennes, Belgium. Nat Hazards Earth Syst Sci 9:507-521.

Van Westen C, Rengers N, Soeters R (2003) Use of geomorphological information in indirect landslide susceptibility assessment. Natural Hazards 30:399-419.

Van Westen C, Van Asch TW, Soeters R (2006) Landslide hazard and risk zonationwhy is it still so difficult? Bulletin of Engineering geology and the Environment 65:167-184.

Van Westen CJ (2000) The modeling of landslide hazards using GIS. Surveys in Geophysics 21:241-255. doi:10.1023/a:1006794127521

van Westen CJ, Castellanos E, Kuriakose SL (2008) Spatial data for landslide susceptibility, hazard, and vulnerability assessment: An overview. Engineering Geology 102:112-131. doi:10.1016/j.enggeo.2008.03.010

Van Westen CJ, Rengers N, Soeters R (2003) Use of geomorphological information in indirect landslide susceptibility assessment. Natural Hazards 30:399419. doi:10.1023/B:NHAZ.0000007097.42735.9e 
Van Westen CJ, Rengers N, Terlien MTJ, Soeters R (1997) Prediction of the occurrence of slope instability phenomena through GIS-based hazard zonation. International Journal of Earth Sciences 86:404-414.

Van Westen CJ, Terlien MTJ (1996) An approach towards deterministic landslide hazard analysis in GIS. A case study from Manizales (Colombia). Earth Surface Processes and Landforms 21:853-868. doi:10.1002/(sici)10969837(199609)21:9<853::aid-esp676>3.0.co;2-c

van Westen CJ, van Asch TWJ, Soeters R (2006) Landslide hazard and risk zonation - Why is it still so difficult? Bulletin of Engineering Geology and the Environment 65:167-184. doi:10.1007/s10064-005-0023-0

Varnes D (1984) IAEG Commission on Landslides \& other Mass Movements. Landslide hazard zonation: a review of principles and practice 63.

Varnes DJ (1978) Slope movement and types and processes. Landslides: Analysis and Control:11-33.

Varnes DJICoLaoM-M (1984) Landslide hazard zonation: a review of principles and practiceUNESCO Press. Paris (1984) 63 pp.

Vaughn NR, Asner GP, Smit IPJ, Riddel ES (2015) Multiple Scales of Control on the Structure and Spatial Distribution of Woody Vegetation in African Savanna Watersheds. PLoS ONE 10:e0145192. doi:10.1371/journal.pone.0145192.

Weiss A Topographic position and landforms analysis. In: Poster presentation, ESRI user conference, San Diego, CA, 2001.

Wood JL, Harrison S, Reinhardt L (2015) Landslide inventories for climate impacts research in the European Alps. Geomorphology 228:398-408. doi:http://dx.doi.org/10.1016/j.geomorph.2014.09.005

Xiao H, Huang J, Ma Q, Wan J, Li L, Peng Q, Rezaeimalek S (2015) Experimental study on the soil mixture to promote vegetation for slope protection and landslide prevention. Landslides:1-11. doi:10.1007/s10346-015-0634-X

Xu C (2015) Preparation of earthquake-triggered landslide inventory maps using remote sensing and GIS technologies: Principles and case studies. Geoscience Frontiers 6:825-836.

doi:https://doi.org/10.1016/j.gsf.2014.03.004

Yeon YK, Han JG, Ryu KH (2010) Landslide susceptibility mapping in Injae, Korea, using a dicision tree, Engineering Geology 116:274-283.

doi:10.1016/j.enggeo.2010.09.009

ZHANG Y, Wei-ya X, Li-fangi Z (2013) Analysis of seepage stability of large-scale landslide under rainfall Condition. Rock and Soil Mechanics 34:833-841.

Ziemer RR (1981) The role of vegetation in the stability of forested slopes. 


\section{Summary}

Landslides susceptibility modelling is a crucial step towards the assessment of landslide hazard and risk aiming at reducing the casualties, economic losses and environmental damages of landslides. Over the last decades, landslide susceptibility modelling has been a field of research for many researchers to try different new data integration techniques and approaches. In this context, developments of commercial, open source GIS and statistical software and availability of environmental data have facilitated landslide susceptibility modelling using variety quantitative approaches. In these approaches, relations between distribution of landslides and environmental factors are explored using the assumption that future landslides will more likely occur under environmental conditions leading to the occurrence of landslides in the past. This thesis focuses on a fundamentally different viewpoint: the effects of landslides on susceptibility of future landslides. In a sense that landslides will change the likelihood of the occurrence of future landslides through their effects on the environment. This is called landslide path dependency in this thesis. Exploring such an effect needs landslides to be documented and mapped regularly to ensure that multi-stage activities of landslides are properly monitored. Different indexes and methods are developed and evaluated to: (i) explore whether landslides following previous landslides, (ii) compare differences between landslides that follow landslides and landslides that do not, (iii) quantify the spatiotemporal scales of landslide path dependency, and (iv) assess the impacts of landslide path dependency on slope unit-based and pixel-based landslide susceptibility modelling.

After introducing the general objective of the thesis in Chapter 1, Chapter 2 explores the indications of path dependency among landslides in a multi-temporal landslide inventory from the Collazzone study area in Umbria in central Italy. Spatial overlap was used as an index to explore landslide path dependency effect. Differences in size, shape and parameters of power-law distribution were compared between landslides that follow previous landslides and landslides that do not. The overlap index among landslide was found to remain high for about a decade and decreases significantly over time. Follow-up landslides were larger and more elongated than non-follow-up landslides. Power-law behaviour was found in both follow-up landslides and no-follow-up landslides. A modified time-variant landslide susceptibility was proposed reflecting the spatiotemporal effects of landslide path dependency.

In Chapter 3, the strength and duration of landslide path dependency was quantified using the follow-up landslide fraction. This showed that susceptibility for a new follow-up landslide increased by a factor of 15 immediately after a landslide, and that this extra susceptibility decayed exponentially over time. 
Geometric and topographic properties of landslides with and without follow-up landslides were compared and based on that, the occurrence of follow-up landslides was predicted by logistic regression. Larger and rounder landslides were found to facilitate the occurrence of follow-up landslides. Also, landslides in wetter places and in lower slope positions are more likely to cause follow-up landslides. Field exploration of landslides also revealed that changes in slope hydrology can contribute to the occurrence of follow-up landslides. A combination of geometric and topographic properties of landslides predicted the occurrence of follow-up landslide acceptably well. Including the geometry of landslides leads to a better performance of models predicting follow-up landslide as compared to the situation when only topographic properties were used.

In Chapter 4, the landslide path dependency effect was implemented in slope unitbased landslide susceptibility modelling. Three models were developed using logistic regression: (i) a conventional landslide susceptibility model constructed with 51 conditioning attributes, (ii) a conventional plus path dependent landslide susceptibility model constructed with 51 conditioning attributes plus two landslide path dependency variables and (iii) path dependent landslide susceptibility model constructed only with two landslide path dependency variables. The conventional plus path dependent susceptibility had slightly better model performance than conventional susceptibility but the differences in their performances were not statistically significant. The resulting landslide susceptibility maps were similar except for slight differences in a few slope units.

In Chapter 5, landslide path dependency was quantified in a point-based multitemporal landslide inventory. Ripley's space-time K Function was used to quantify the degree of clustering among landslides. The resulted clustering measure revealed that landslides tend to cluster in 200 meter distance from a previous landslide in the time scale of a decade with an exponential decay over time. Such an effect was implemented in a pixel-based landslide susceptibility modelling. Again, three models were developed using logistic regression : (i) a conventional landslide susceptibility constructed with Digital Elevation Model-derivatives, (ii) a conventional plus path dependent landslide susceptibility with DEM-derivatives and two landslide path dependency variables and (iii) path dependent landslide susceptibility only with two landslide path dependency variables. Model performance of conventional landslide susceptibility improved considerably with adding landslide path dependency effects. In fact, even the purely path dependent landslide susceptibility model performed better than the conventional landslide susceptibility model.

Chapter 6 summarizes the main finding of the thesis, concludes the thesis, and provides additional implications and prospects for future research. By quantifying the spatiotemporal landslide path dependency, the effects of landslides on future susceptibility can now be considered in landslide susceptibility modelling. Such an effect provides an underlying concept towards the dynamic time-variant landslide susceptibility modelling. In this context, multi-temporal landslide inventory was therefore able to provide valuable information to monitor the multi-stage activities 
of landslides. However, the work presented only explores landslide path dependency in the Collazzone study area in Italy, future research should focus on the existence and quantification of landslide path dependency in other landslide prone areas. This needs further regular monitoring and multi-temporal mapping of landslides. The advance in high spatial and temporal resolutions of remote sensing imagery could facilitate preparation of multi-temporal landslide inventories the coming decades. 


\section{Acknowledgement}

My dream came true eventually, doing PhD in the field of GIS in an international highly-ranked university in the Netherlands. Yes, I did it! All tough, frustrating and also nice moments in this scientific journey reminds me of the famous Persian poem

$$
\text { "حويند سنى لعل شود در مقام صبر آرى شود وليك به خون جكر شود" }
$$

I would not have been able to overcome the challenges emerged in this journey without the support of several nice people, and I would like to truly thank them for keeping me motivated to end this journey.

First and foremost, I would like to state my sincere thanks to my promoter, Prof Arnold Bregt, who gave me the opportunity to pursue my PhD at the Laboratory of Geo-Information Science and Remote Sensing of Wageningen University. Arnold, you have been the kindest person I have ever seen in my whole life. I am very thankful for you guidance, inspiration, support and also trust that you gave me during this long and sometimes winding path.

I would also like to appreciate the motivation and support that were given to me by my Promoter Prof Jakob Wallinga. Jakob, I am truly grateful for all your supports, constructive ideas and comments during the whole PhD.

If I say that Dr Arnaud Temme as my daily supervisor was and is the most influential person in my scientific career, I am not exaggerating. Arnaud, I have been thinking and looking for words and sentences to express how grateful I am from your unbelievable supports in my PhD. I still remember the first day that I came to your office and talked to you about my project and how enthusiastic you were about my topic. That day, I told to myself that this is what I am exactly looking for, you were a great companion in my scientific journey. You may not remember the other day that you came to my office to inform me that you are moving to Kansas State University, US. I was truly sad but one of your sentences is still in my ears, "do not worry Jalal, I will be committed to your success", and that kept me motivated to continue working with you regularly and remotely via skype. I also would like to thank you for all your support that you have provided for me and my family during our stay in Manhattan, USA.

I would like to thank all my colleagues from the laboratory of Geo-Information Science and Remote Sensing. A few people I would like to mention in particular; my great office mates: Lala, Marston and Eskender, and all the colleagues and PhD candidates in the group: Aldo, Sytze, Willy, Lammert, Ron, Marcello, Arend, Frans, John, Devis, Jan, Corné, Roland, Antoinette, Truus, Ben, Mathieu, Sarah, Kim, Johannes, Jose, Nandika, Michi, Giulia, Richard, Niki, Arun, Patric, Roberto, Brice, Erika, Kalkidan, Astrid, Yang, Simon, Tsoefiet, Valerio, Rosa, Alvaro, Qijun, Maria, Konstantin, Sabina, Marian, Danaë, Joao, Daniela, Eliakim, and Benjamin.

All my Iranian friends are thanked for their support during my PhD. We had a lot of fun in our family gatherings in the weekend and hope to repeat these nice moments 
in Iran. For this, first I would like to thank Mansoor and Farzaneh for their unbelievable hospitality in the time of our entrance into the Netherlands. I also would like to extend my gratitude to Saeed and Maryam, Sasan and Maryam for their great support and guidance during the first couple of months. Also many thanks to Arash, Naser, Amir, Mohammad, Mahyar, Farsheed for the nice atmosphere during our football games.

I would also like to appreciate the faculty members of Geography department in the University of Mazandaran, Iran. Special thanks to Dr Jokar as my study coordinator for all the support. Very special thanks to Dr Mirnia, the dean of university of Mazandaran, for all your unbelievable support during my $\mathrm{PhD}$ and I am truly grateful for that.

Three of my best friends in Iran, Dr Seyedi, Dr Bagheri and Dr Masoodi, I am very much grateful for your support and perusing my officials work in Iran. Also, my special thanks goes to the supervisor of my MSc period, Prof A.K Matkan: It has been an honor to start my scientific journey with you and I am very grateful for all your endless support, guidance and trust.

Very special thanks to the two of my best family friends Arman and Nafiseh \& Mehdi and Narges. We had a lot of fun most of the weekends in the last couple of years, and I really would like to thank you for all the nice moments that you provided for my family so that we felt at home in the Netherlands. Narges and Nafiseh, you do know that I have a sister but I believe that I already have three sisters since you provided all the support that my sister would provide for me and I am really grateful for that. Mehdi, wish you all the best in your life with Narges, and would like to thank you for helping me to design the cover of my PhD thesis. But Arman, my best friend in the Netherlands, you were and are also like my brother, all the motivation, guidance and support from your side are truly appreciated and I wish our friendship last forever.

A big thankyou to my dear parents, Baba Jalil and Maman Farangis, whatever I have and I have reached so far, I believe that is for many years of your endless support and encouragement throughout my life, and I wish both of you stay healthy forever. My lovely Baba and Maman, I missed you so much and I am counting the days to come back and kiss your hands. I also would like to thank my lovely brothers, Hasan, Javad, Hamed and Hossein and my dear pretty sister Arezou for all your support throughout my PhD. Dear Arezou, our regular weekly skype would give me a big motivation to endure the difficulties of being away from my loved ones and for that I would like to appreciate. I am also very grateful to the members of my extended family, Parisa, Bahar, and my lovely pretty nephew Anisa. A big thankyou also goes to my father-in-law family, Pedar Nemat and Madar Zahra, Reza, Shamim and lovely little Baran. Maman Shahnaz, with love and remembrance, god bless you, you were truly one of my great supporters in my life, you always would want me to reach to the highest academic degree, today I have reached to your wish and I am pretty sure that you are aware of that. 
And my lovely wife, Shaghayegh, words and sentences cannot express how grateful I am for your endless love, patient, support and kindness throughout my life. My dear, truly I was not able to reach to this achievement without having the best companion as you, and I am thankful for that. My little man, my dear son, Mohammadtaher, your smiles and playful eyes were the best ever motivation for me to overcome all the challenges in life, and I hope and pray for you to achieve to whatever you want in your life.

Thank you all!

Jalal

Wageningen, 19. September 2018 


\section{List of publications}

- Samia J, Temme A, Bregt A, Wallinga J, Stuiver J,Guzzetti F, Ardizzone F, Rossi M (2018), Implementing landslide path dependency in landslide susceptibility modelling, Landslides, DOI 10.1007/s10346-018-1024-y

- Samia J, Temme A, Bregt A, Wallinga J, Guzzetti F, Ardizzone F, Rossi M (2017), Characterization and quantification of path dependency in landslide susceptibility, Geomorphology, 292, 16-24,

DOI.org/10.1016/j.geomorph.2017.04.039

- Samia J, Temme A, Bregt A, Wallinga J, Guzzetti F, Ardizzone F, Rossi M (2016), Do landslides follow landslides? Insights in path dependency from a multi-temporal landslide inventory, Landslides, 14, 547-558, DOI:10.1007/s10346-016-0739-X

\section{Other scientific contributions}

- $\quad$ Samia J, Temme A, Bregt A, Wallinga J, Guzzetti F, Ardizzone F, Rossi M (2017), Path dependent landslide susceptibility modelling, Netherland Centre for Geodesy and Geo-information (NCG), Delft University of Technology, The Netherlands

- Samia J, Temme A, Bregt A, Wallinga J, Guzzetti F, Ardizzone F, Rossi M (2017), Quantifying landslide path dependency, American Association of Geographers (AAG), Boston, USA

- Samia J, Temme A, Bregt A, Wallinga J, Guzzetti F, Ardizzone F, Rossi M (2017), Why landslide susceptibility maps should change over time, Association of Geographic Information Laboratories in Europe (AGILE), Wageningen University and Research, The Netherlands

- $\quad$ Samia J, Temme A, Bregt A, Wallinga J, Guzzetti F, Ardizzone F, Rossi M (2016) Do landslides follow landslides?, Geophysical Research Abstracts, Vol. 18, European Geosciences Union (EGU) 2016-125, Vienna, Austria

- $\quad$ Temme A, Samia J, Nijp J, Meij M, Masselink R (2016), The generic danger and the idiosyncratic support, Geophysical Reasearch Abstarct, Vol. 18, European Geoscience Union (EGU) 2016-18033, Vienna, Austria 


\section{Short biography}

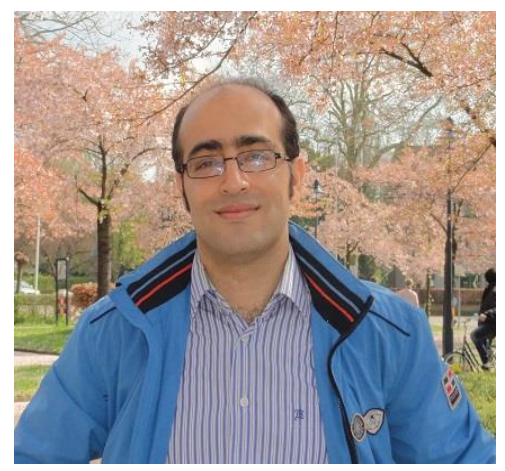

Jalal Samia was born in Amol, Iran, on September 21, 1980. Growing up in the amazing landscape of north of Iran - close by to Caspian Sea and Damavand Peak he developed an interest in environmental sciences. He obtained his bachelor in 2003, in the field of Natural Resource Engineering from Agricultural Sciences and Natural Resources University of Gorgan, Iran. During this period, he became interested in the applications of aerial photography, remote sensing and GIS for environmental issues. This passion encouraged him to study GIS and Remote Sensing at Shahid Beheshti University, Tehran, Iran, with the rank of 10 in the national exam. In 2007, he obtained his master degree. During this period, he also worked as GIS specialist at Naghsh Click International Company in Tehran.

From 2007 to 2013, he worked as a faculty member of Payam Noor University of Mazandaran, and was involved in teaching on GIS and Remote Sensing related courses. In 2013, he was awarded a scholarship from the Ministry of Science, Research and Technology of Iran to pursue his PhD at Laboratory of GeoInformation Science and Remote sensing of Wageningen University \& Research in the Netherlands. Besides being involved in the Laboratory of GIS and Remote Sensing, he also worked closely with Soil Geography and Landscape Group of Wageningen University and the Research Institute for Geo-Hydrological Protection (CNR-IRPI) in Italy. Jalal worked on his last chapter of his PhD thesis in the Geography Department of Kansas State University, USA. During his PhD, Jalal has published three peer-review publications as first author and one paper which is currently under review. He also presented his research at several international and national conferences both oral and poster presentation. He also won the PE\&RC call for short term and small scale strategic projects in 2018, and is currently working as a postdoc in the Laboratory of GIS and Remote Sensing of Wageningen university and research. 
With the training and education activities listed below the PhD candidate has complied with the requirements set by the C.T. de Wit Graduate School for Production Ecology and Resource Conservation (PE\&RC) which comprises of a minimum total of 32 ECTS (= 22 weeks of activities)

\section{Review of literature (6 ECTS)}

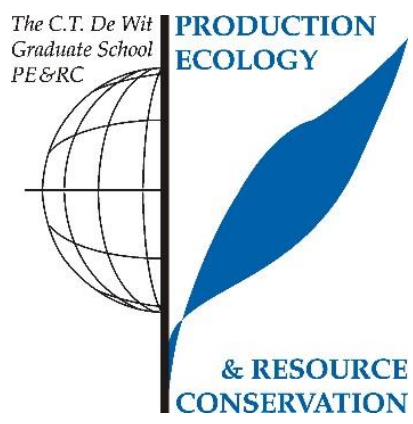

- Landslide susceptibility modelling

\section{Writing of project proposal (4.5 ECTS)}

- $\quad$ Path dependent spatial and temporal modelling of landslide susceptibility

\section{Post-graduate courses (6.6 ECTS)}

- $\quad$ Art of modelling; PE\&RC (2015)

- Geostatistics; PE\&RC (2015)

- Uncertainty propagation in spatial environmental modelling; PE\&RC (2016)

- Introduction to R for statistical analysis; PE\&RC (2016)

\section{Laboratory training and working visits (0.9 ECTS)}

- Investigating the role of path dependency in landslide susceptibility modelling; CNR IRPI, Perugia, Italy (2016)

\section{Invited review of (unpublished) journal manuscript (1 ECTS)}

- Journal of soils and sediments: the effect of landslide on soil organic carbon stock and biochemical properties of soil (2017)

\section{Deficiency, refresh, brush-up courses (18 ECTS)}

- Spatial data infrastructure; Laboratory of GIS and Remote Sensing (2014)

- Spatial modelling and statistics; Laboratory of GIS and Remote Sensing (2014)

- Advanced geo-information science for earth and environment; Laboratory of GIS and Remote Sensing (2014)

\section{Competence strengthening / skills courses (4.8 ECTS)}

- PhD Competence assessment; WGS (2014)

- The essential of scientific writing and presenting; WGS (2015)

- $\quad$ Scientific writing; WGS (2016)

- $\quad$ Project and time management; WGS (2017) 
PE\&RC Annual meetings, seminars and the PE\&RC weekend (3.3 ECTS)

- $\quad$ PE\&RC Weekend (2013)

- $\quad$ PE\&RC Day (2013)

- PE\&RC Symposium ( The search for tipping points in heterogeneous landscape) (2013)

- $\quad$ Midterm PE\&RC PhD weekend (2014)

- Symposium “Complex Adaptive Systems” in Wageningen (2015)

- $\quad$ PhD Workshop carousel (2015)

- $\quad 3^{\text {rd }}$ Wageningen PhD symposium "Diversity in Science" (2016)

- Symposium "Frontiers in resilience research" (2017)

Discussion groups / local seminars / other scientific meetings (7.5 ECTS)

- $\quad$ Modelling and Statistic Network (MSN) (2013-2016)

- $\quad$ R User meeting (2016-2018)

International symposia, workshops and conferences (9.2 ECTS)

- European Geoscience Union (EGU) international conference; Vienna, Austria; oral presentation (2016)

- Association of American Geographers (AAG); Boston, USA; oral presentation (2017)

- Association of Geographic information Laboratories in Europe (AGILE); Wageningen, the Netherlands; poster presentation (2017)

- Netherlands Centre for Geodesy and Geo-Informatics (NCG) symposium; University of Delft, the Netherlands; oral presentation (2017)

\section{Supervision of MSc students (6 ECTS)}

- $\quad$ Spatial modelling of landslide susceptibility

- Exploring requirements for creating multi-temporal landslide inventory using remote sensing 
This work has been conducted at Laboratory of Geo-Information Science and Remote Sensing and Soil Geography and Landscape group of Wageningen University and Research in the Netherlands, and was financially supported by the Ministry of Science, Research and Technology of Iran.

Thesis layout: Jalal Samia

Cover design: Samad Bahrami Kashkooli, kashkoolisamad@gmail.com

Printed by: ProefschriftMaken, || www.proefschriftmaken.nl 